\title{
TWO-GROUP CONSTANTS FOR REACTOR MATERIALS
}

By

Mary J. Stanley

May 1958

Aircraft Nuclear Propulsion Department General Electric Company

Cincinnati, Ohio

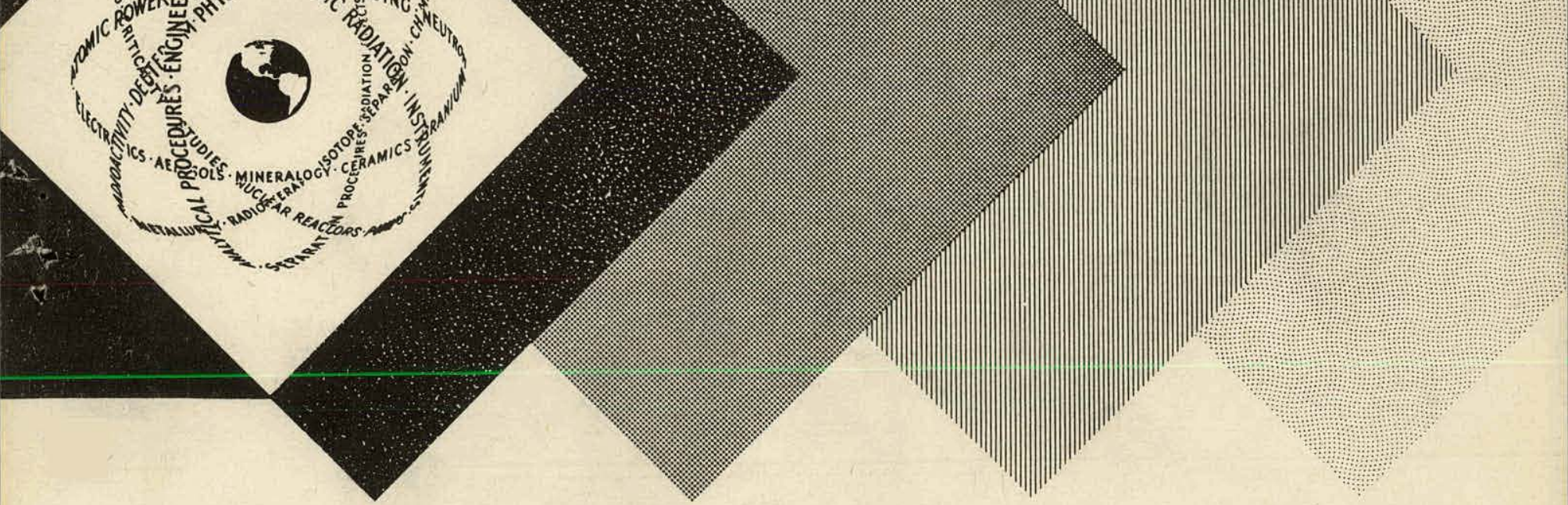




\section{DISCLAIMER}

This report was prepared as an account of work sponsored by an agency of the United States Government. Neither the United States Government nor any agency Thereof, nor any of their employees, makes any warranty, express or implied, or assumes any legal liability or responsibility for the accuracy, completeness, or usefulness of any information, apparatus, product, or process disclosed, or represents that its use would not infringe privately owned rights. Reference herein to any specific commercial product, process, or service by trade name, trademark, manufacturer, or otherwise does not necessarily constitute or imply its endorsement, recommendation, or favoring by the United States Government or any agency thereof. The views and opinions of authors expressed herein do not necessarily state or reflect those of the United States Government or any agency thereof. 


\section{DISCLAIMER}

Portions of this document may be illegible in electronic image products. Images are produced from the best available original document. 
Date Declassified: November 4, 1959.

\section{LEGAL NOTICE}

This report was prepared as an account of Government sponsored work. Neither the United States, nor the Commission, nor any person acting on behalf of the Commission:

A. Makes any warranty or representation, expressed or implled, with respect to the accuracy, completeness, or usefulness of the information contained in this report, or that the use of any information, apparatus, method, or process disclosed in this report may not infringe privately owned rights; or

B. Assumes any liabilities with respect to the use of, or for damages resulting from the use of any information, apparatus, method, or process disclosed in this report.

As used in the above, "person acting on behalf of the Commission" includes any employee or contractor of the Commission, or employee of such contractor, to the extent that such employee or contractor of the Commission, or employee of such contractor prepares, disseminates, or provides access to, any information pursuant to his employment or contract with the Commission, or his employment with such contractor.

This report has been reproduced directly from the best available copy.

Printed in USA. Price $\$ 1.50$. Avallable from the Office of Technical Services, Department of Commerce, Washington 25, D. C.

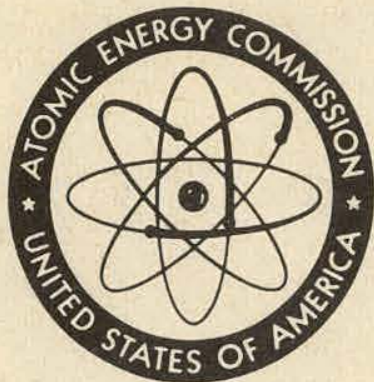


APEX-369

\title{
TWO-GROUP CONSTANTS FOR REACTOR MATERIALS
}

\author{
Mary J. Stanley \\ Nuclear Dovelopment \\ Laboratories Sub-Section
}

Moy 1958

United Stotes Air Force

Unifed States Atomic Energy Commission
Coniract No. AF 33(038)-21102

Contract No. AT $(11-1)-171$

\author{
GENERAL ELECTRIC \\ AIRCRAFT NUCLEAR PROPULSION DEPARTMENT \\ ATOMIC PRODUCTS DIVISION \\ Cincinnati 15, Ohio
}




\section{ACKNOWLEDGMENTS}

The author wishes to thank W. B. Henderson for tabulation of basic cross section data from which the two-group constants were computed and for his invaluable assistance in the preparation of this report. The author also wishes to thank Florence Silverstein for the $U^{235}$ fission spectrum used in the age calculations. 


\section{ABSTRACT}

In order to facilitate reactor design studies a compilation of calculated two-group constants averaged over the infinite-medium flux produced by a fission source has been made for approximately 80 materials of interest to reactor engineers. A comparison with available experimental age meas urements is included. 


\section{CONTENTS}

\section{Page}

1. Glossary of Terms $\ldots \ldots \ldots \ldots \ldots \ldots \ldots$

2. Theory . . . . . . . . . . . . 7

3. Use of the Two-Group Constants . . . . . . . . 10

4. Tabulation of Two-Group Constants .......... 11

5. Comparison With Experiment . . . . . . . . . 59

6. References ..................61 


\section{GLOSSARY OF TERMS}

The following is a list of terms, subscripts, and abbreviations used in this report.

\section{NOME NCLAT URE}

$d$ extrapolation distance

$D$. diameter of cylinder; diffusion coefficient

$H$ height of cylinder

$k$ neutron multiplication constant

$K^{2}$ buckling ,

$L$ leakage spectrum

$L^{2}$ diffusion area

$M^{2}$ migration area

$p$ absorption-escape probability

$q$ slowing-down density

$S$ source spectrum; reflector savings

$u \quad$ lethargy, $u=\ln \frac{E_{0}}{E}$ where: $E=$ neutron energy, ev, and $E_{0}=10^{7} \mathrm{ev}$

$U$ lethargy interval, $U_{n}=u_{n}-u_{n-1}$

$\phi$ flux

$\xi$ mean rise in lethargy per scattering collision

$\Sigma_{a}$ macroscopic absorption cross section

$\Sigma$ macroscopic fission cross section

$\Sigma_{l}$ macroscopic leakage cross section

$\Sigma_{s}$ macroscopic slowing-down cross section

$\Sigma_{t r}^{s}$ macroscopic transport cross section

7 age

$\nu$ number of neutrons per fission

\section{SUBSCRIPTS}

In indium resonance of $1.4 \mathrm{ev}$

$n$ lethargy index

$t$ last-epithermal-lethargy index

$t+1$ thermal-group index

Th thermal energy

$r, z$ space coordinates

1 fast-group designation

2 thermal-group designation 
ABBREVIATIONS USED IN SECTION 4

HCP: Hodgman, Charles D. Handbook of Chemistry and Physics, 37 Edition, Chemical Rubber Publishing Company, Cleveland, Ohio, 1955-1956.

MH: Lyman, Taylor, Metals Handbook, The American Society for Metals, Cleveland, Ohio, 1948.

RH: The Reactor Handbook, Volume I, Physics, U.S. Atomic Energy Commission, RH-1, June 1953.

RMH: Hampel, Clifford A., Rare Metals Handbook, New York, Reinhold Publishing Corporation, 1954.

Campbell, Ivor.E., High-Temperature Technology, New York, Wiley Publishing Company, 1956, p 31.

Everhart, John L., Mechanical Properties of Metals and Alloys, Washington, D.C., Government Printing Office, Bureau of Standards circular, C447, 1943.

GE-ANPD, Internal Communications, General Electric Aircraft Nuclear Propulsion Department.

Callihan, D., and Keen, R. C., Critical Experiments on Direct Cycle Aircraft Reactor, Oak Ridge National Laboratories, ORNL-1615, November 13, 1953.

6 


\section{THEORY}

In order to facilitate reactor design studies a compilation of two-energy-group parameters, commonly called two-group constants, $D_{1}, D_{2}, \Sigma_{a_{1}}, \Sigma_{a_{2}}, d, d_{1}, d_{2}, L_{1}^{2}, L_{2}^{2}, \Sigma_{s}, p, i \Sigma_{f_{1}}, \nu \Sigma_{f_{2}}, k_{1}$, and $k_{2}$ was made for various reactor materials.

A program that utilizes a multi-energy diffusion theory $\left(C_{2}-\text { Short }\right)^{11}$ for a bare cylindrical reactor was used to obtain two-group constants for an infinite medium of each material. A cylinder with a radius of $10^{6}$ centimeters and a height of $10^{6}$ centimet ers was used to obtain an essentially infinite medium; buckling is then a very small nonzero value. The results are tabulated for Fahrenheit temperatures of $68^{\circ}, 500^{\circ}, 1000^{\circ}, 1500^{\circ}, 2000^{\circ}, 2500^{\circ}$, and $3000^{\circ}$. The division of the two groups is at thermal le thargy. The two-group constant $s$ were computed for the density of the material used at GE-ANPD whenever this dat a was available; othe rw ise handbook values were used. In some cases densities other than the one used are also quoted for the information of the reader.

Modified age the ory ${ }^{12}$ was used in calculat ing two-group constants for all the materials. This seems to be the best slowing-down model for engineering use at the present time. (See section 5. )

The multi-energy cross sections used by Program $\mathrm{C}_{2}$-Short are on an ANPD nuclear-data tape and were calculated from basic cross section data.

In Program $\mathrm{C}_{2}$-Short the neutron conservation equation:

$$
\frac{d q}{d u}=S(u)-\left[\Sigma_{a}(u)+D(u) K^{2}(u)\right] \phi(u)
$$

where:

$$
\begin{array}{ll}
q & =\text { slowing-down dens ity } \\
u & =\text { lethargy } \\
S(u) & =\text { source } \\
\Sigma_{a} & =\text { macroscopic absorption cross section } \\
D & =\text { diffusion coefficient } \\
K^{2} & =\text { buckling } \\
\phi & =\text { flux }
\end{array}
$$

is solved for $q$ at each lethargy division in the following manner. The modified age treatment yields the equation:

$$
\phi(u)=\frac{q(u)+S(u)}{\xi \Sigma_{s}(u)+\Sigma_{a}(u)+D(u) K^{2}(u)}
$$

as the relation between flux and slowing-down density. Equation (2) is used to eliminate $\phi(u)$ in equation (1). The resultant first-order differential equation in $q$ is integrated trapezoidally between successive lethargy divisions.

The lethargy divisions are at $u=0,0.5,1.0,1.5,2.0,2.5,3.0,3.5,4.0,6.0,8.0,10.0,12.0,14.0$, $15.5,16.5,17.5,18.5,19.795$.

From the resultant flux and slowing-down density, the two-group constants for fast and thermal groups are calculated as indicated on the following pages. 
$\phi_{1}=\operatorname{Sum}_{n=1}^{t}\left(\phi_{n}+\phi_{n-1}\right) \frac{U_{n}}{2}=$ fast-group slux, where $t$ is the last-epithermal index.

$\Sigma_{a_{1}}=\frac{1}{\phi_{1}} \operatorname{Sum}_{n=1}^{t}\left(\Sigma_{a_{n}} \phi_{n}+\Sigma_{a_{n-1}} \phi_{n-1}\right) \frac{U_{n}}{2}=$ fast-group macroscopic absorption cross section. (4)

$\nu \Sigma_{f_{1}}=\frac{1}{\phi_{1}} \operatorname{sum}_{n=1}^{t}\left(\nu \Sigma_{f_{n}} \phi_{n}+\nu \Sigma_{f_{n-1}} \phi_{n-1}\right) \frac{U_{n}}{2}=\nu$ times the fast-group macroscopic fission

$k_{1}=\frac{\nu \Sigma_{f_{1}}}{\Sigma_{a_{1}}}=$ fast-group multiplication constant.

$\Sigma_{s_{1}}=\frac{q_{t}}{\phi_{1}}=$. fast-group slowing-down probability per unit path length.

$d_{1}=\frac{1}{\phi_{1}} \operatorname{sum}_{n=1}^{t}\left(d_{n} \phi_{n}+d_{n-1} \phi_{n-1}\right) \frac{U_{n}}{2}=$ fast-group extrapolation distance.

$d_{2}=d_{t+1}=$ thermal-group extrapolation distance.

where $d_{n}=\frac{0.7105}{\left(\Sigma_{a}+\Sigma_{t r}\right)_{n}}, n=0, \ldots, 18, t, t+1$.

$d^{\prime}=\left(\frac{d_{1} \phi_{1}+d_{2} \phi_{2}}{\phi_{1}+\phi_{2}}\right)=$ ave rage extrapolation distance.

$\phi_{2}=\phi_{t+1}=$ thermal-group flux, where $t+1$ is the thermal index.

$\Sigma_{a_{2}}=\Sigma_{a_{t+1}}=$ themal-group macroscopic absorption cross section.

$\nu \Sigma_{f_{2}}=\nu \Sigma_{f_{t+1}}=\nu$ times the thermal-group fission cross section.

$k_{2}=\frac{\nu \Sigma_{f_{2}}}{\Sigma_{a_{2}}}=$ thermal-group multiplication constant.

$P=\frac{\Sigma_{s_{1}}}{\Sigma_{s_{1}}+\Sigma_{a_{1}}}=$ absorption-escape probability.

$D_{1}=\frac{\Sigma_{l_{1}}}{K_{1}^{2}}=$ fast-group diffusion coefficient,

where $\Sigma_{l_{1}}=\frac{1}{\phi_{1}} \operatorname{Sum}_{n=1}^{\imath}\left(\Sigma_{l_{n}} \phi_{n}+\Sigma_{l_{n-1}} \phi_{n-1}\right) \frac{U_{n}}{2}, \Sigma_{\eta_{n}}=D_{n} K_{n}^{2}$,

and $K_{1}^{2}=\frac{9.87}{\left(H+2 S_{Z}+2 d_{1}\right)^{2}}+\frac{23.13}{\left(D+2 S_{+}+2 d_{1}\right)^{2}}$ 
$D_{2}=\frac{\Sigma_{l_{2}}}{K_{2}^{2}}=$ thermal-group diffus ion coefficient,

where $\Sigma_{l_{2}}=\Sigma_{l_{t+1}}$

and $K_{2}^{2}=\frac{9.87}{\left(H+2 S_{z}+2 d_{2}\right)^{2}}+\frac{23.13}{\left(D+2 S_{\tau}+2 d_{2}\right)^{2}}$

$L_{1}^{2}=\frac{D_{1}}{\Sigma_{a_{1}}+\Sigma_{s_{1}}}=$ fast-group diffusion area.

$L_{2}^{2}=\frac{D_{2}}{\Sigma_{a_{2}}}=$ thermal-group diffusion area. 


\section{USE OF THE TWO-GROUP CONSTANTS}

The two-group constants presented here can be us ed as input data in IBM 704 diffusion programs $F_{2}{ }^{11}$ and CURE ${ }^{13}$. Although the two-group constants were calculated for an infinite medium, they may be used in the finite geometry of $\vec{F}_{2}$ and CURE as a first approximation in nonmultiplying regions. The use of these two-group constants will save machine time and engine ering time in evaluating various reactors.

The two-group equations for a space-dependent calculation of reactivity $k$ are:

$$
\begin{aligned}
& -\nabla \cdot D_{1} \nabla \phi_{1}+\left(\Sigma_{a_{1}}+\Sigma_{s_{1}}\right) \phi_{1}=S_{1}\left(\frac{\nu}{k} \Sigma_{f_{1}} \phi_{1}+\frac{\nu}{k} \Sigma_{f_{2}} \phi_{2}\right)+S_{1} \text {, extemal } \\
& -\nabla \cdot D_{2} \nabla \phi_{2}+\Sigma_{a_{2}} \phi_{2}=S_{2}\left(\frac{\nu}{k} \Sigma_{f_{1}} \phi_{1}+\frac{\nu}{k} \Sigma_{f_{2}} \phi_{2}\right)+\Sigma_{s_{1}} \phi_{1}+S_{2} \text {, extemal }
\end{aligned}
$$

The criticality equation that can be derived from (23) and (24) for the case of an infinite medium or a homogeneous reactor with no reflector or extemal sources and group division below the fission spectrum, is:

$$
k=\frac{k_{1}(1-t)}{1+L_{1}^{2} K^{2}}+\frac{k_{2} p}{\left(1+L_{1}^{2} K^{2}\right)\left(1+L_{2}^{2} K^{2}\right)} .
$$

With the assumption of a value for $k$, e.g., $k=1$ for a critical reactor, equation (25) can be sulved for the buckling $K^{2}$. The knowledge of $K^{2}$ enables the optimum dimensions for a bare equivalent react or to be obtained from the equation:

$$
K^{2}=\left(\frac{\pi}{H+2 S_{z}+2 d}\right)^{2}+\left(\frac{2.405}{R+S_{r}+d}\right)^{2}
$$

by finding values of $H$ and $R$ that minimize the core volume $\pi R^{2} H$.

The twa-group constants are also of use in estimating spectra and the rmal-neutron production in shields. 


\section{TABULATION OF TWO-GROUP CONSTANTS}

The two-group constants are listed alphabetically on the following pages. The dimensions of $d, d_{1}$, $d_{2}, D_{1}$, and $D_{2}$ are centimeters $(\mathrm{cm})$; of $\Sigma_{a_{1}}, \Sigma_{a_{2}}, \Sigma_{f_{1}}, \Sigma_{f_{2}}$, and $\Sigma_{s_{1}}$, reciprocal centimeters $\left(\mathrm{cm}^{-1}\right)$; of $L_{1}^{2}$ and $L_{2}^{2}$ square ceitimeters $\left(\mathrm{cm}^{2}\right)$; and of $k_{1}$ and $k_{2}$ are average neutron multiplication. $p$ is a dimensionless fraction. For notations and definitions see sections 1 and 2 respectively. 


\begin{tabular}{|c|c|c|c|c|c|c|c|}
\hline TEMPERATURE: & $\cos F$ & $500 \mathrm{~F}$ & $1000 \mathrm{~F}$ & $15000 \mathrm{~F}$ & $2000 \mathrm{FF}$ & $2500 \% \mathrm{~F}$ & $30000 \mathrm{~F}$ \\
\hline$D_{1}$ & 1.092 & 1.094 & 1.095 & 1.096 & 1.097 & 1.097 & 1.098 \\
\hline$D_{2}$ & .9319 & .9741 & 9967 & 1.009 & 1.017 & 1.023 & 1.026 \\
\hline$\Sigma_{a,}$ & .0007826 & .0006458 & .0005579 & .0005006 & .0004609 & .0004304 & .0004061 \\
\hline$\Sigma_{a_{2}}$ & .009584 & .007108 & .005760 & .004975 & .004439 & .004044 & .003744 \\
\hline$\Sigma_{s_{1}}$ & .001369 & .001545 & .001667 & .001751 & .001812 & .001862 & .001902 \\
\hline$d^{\prime}$ & 2.284 & 2.286 & 2.288 & 2.288 & 2.289 & 2.289 & 2.289 \\
\hline$d_{1}$ & 2.327 & 2.332 & 2.335 & 2.336 & 2.338 & 2.339 & 2.340 \\
\hline$d_{2}$ & 1.986 & 2.076 & 2.124 & 2.151 . & 2.168 & 2.180 & 2.188 \\
\hline$L \frac{2}{1}$ & 507.5 & 499.2 & 492.3 & 486.7 & 482.4 & 478.7 & 475.5 \\
\hline$L_{2}^{2}$ & 97.24 & 137.0 & 173.0 & 202.9 & 229.1 & 252.9 & 274.2 \\
\hline 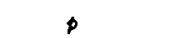 & .6362 & .7053 & .7492 & .7777 & .7973 & .8122 & .8241 \\
\hline
\end{tabular}

COMPOSITION BY WEIGHT FRACTION:

REFERENCE:

$\begin{array}{lr}\text { Al } & .5292 \\ \text { O } & 4708\end{array}$
MATERIAL: 2S Aluminum
DENSITY VARIATIONS AND REFERENCE:

I. E. Campbell, High Temperature Technology, 1954.P. 31 $3.97 \mathrm{gm} / \mathrm{cm}^{3}$

ASSUMED DENSITY: $.2 .700 \mathrm{gm} / \mathrm{cm}^{3}$

\begin{tabular}{|c|c|c|c|c|c|c|c|}
\hline TEMPERATURE: & $60 F$ & $500 \mathrm{~F}$ & $1000 \%$ & $1500 \mathrm{~F}$ & $2000 \mathrm{~F}$ & $2500 \mathrm{~F}$ & $3000 \mathrm{~F}$ \\
\hline$D_{1}$ & 3.858 & 3.859 & 3.859 & 3.859 & 3.860 & 3860 & 3.860 \\
\hline$D_{2}$ & 3.366 & 3.459 & 3.528 & 3.573 & 3.605 & 3.630 & 3.650 \\
\hline & & & - & & & & \\
\hline$\Sigma_{a_{1}}$ & .0004613 & .0004348 & .0004122 & .0003948 & .0003809 & .0003691 & .0003587 \\
\hline$\Sigma_{a_{2}}$ & .01230 & .009122 & .007392 & .006385 & .005698 & .005191 & .004805 \\
\hline$\Sigma_{s_{1}}$ & .00006081 & .00008886 & .0001130 & .0001321 & .0001474 & .0001605 & .0001721 \\
\hline$d$ & 8.219 & 8. 217 & 8.216 & 8.213 & 8.213 & 8.213 & 8.213 \\
\hline$d_{3}$ & $8.224^{\circ}$ & 8.225 & 8.226 & 8.226 & 8.227 & 8.228 & 8.228 \\
\hline$d_{2}$ & 7.174 & 7.372 & 7.519 & 7.615 & 7.684 & 7.738 & 7.780 \\
\hline$L \frac{2}{1}$ & 7389 & 7369. & 7348. & 7325. & 7306 . & 7289. & 7273. \\
\hline$L_{2}^{2}$ & 273.7 & 379.2 & 477.3 & 559.6 & 632.8 & 699.3 & 759.6 \\
\hline$p$ & .1165 & .1697 & .2151 & .2507 & .2790 & .3031 & .3242 \\
\hline
\end{tabular}

COMPOSITION BY WEIGHT FRACTION:

$$
\text { Al } 1.00
$$

REFERENCE:
DENSITY VARIATIONS AND REFERENCE:

$\begin{array}{ll}\mathrm{HCP} & 2.699 \mathrm{gm} / \mathrm{cm}^{3} \\ \mathrm{RH} & 2.699 \mathrm{gm} / \mathrm{cm}^{3}\end{array}$

ANPD: E. A. Aitken

$2.70 \mathrm{gm} / \mathrm{cm}^{3}$ 


\begin{tabular}{|c|c|c|c|c|c|c|c|}
\hline TEMPERATURE: & $60 \% \mathrm{~F}$ & $500 \mathrm{~F}$ & $1000 \% \mathrm{~F}$ & $1500 \% \mathrm{~F}$ & $2000 \mathrm{~F}$ & $25000 \mathrm{~F}$ & $3000 \% \mathrm{~F}$ \\
\hline$D_{1}$ & 3.276 & 3.277 & 3.277 & 3.277 & 3.277 & 3.277 & 3.278 \\
\hline$D_{2}$ & 1.367 & 1.558 & 1.691 & 1.783 & 1.854 & 1.915 & 1. 968 \\
\hline$\Sigma_{a}$ & .0001363 & .0001350 & .0001336 & .0001324 & .0001312 & .0001302 & .0001292 \\
\hline$\Sigma_{a_{2}}$ & .01593 & .01181 & .009578 & .008267 & .007378 & .006725 & .006222 \\
\hline$\Sigma_{s_{3}}$ & $1.927 \times 10^{-6}$ & $3.314 \times 10^{-6}$ & $4.659 \times 10^{-6}$ & $5.983 \times 10^{-6}$ & $7.136 \times 10^{-6}$ & $8.195 \times 10^{-6}$ & $9.184 \times 10^{-6}$ \\
\hline$d$ & 6.983 & 6.983 & 6.983 & 6.982 & 6.982 & 6.982 & 6.982 \\
\hline$d_{1}$ & 6.984 & 6.984 & 6.984 & 6.985 & 6.985 & 6.986 & 6.986 \\
\hline$d_{2}$ & 2.914 & 3.322 & 3.605 & 3.801 & 3.952 & 4.081 & 4. 196 \\
\hline$L_{1}^{2}$ & 23700 . & 23690. & 23690. & 23690. & 23690. & 23680. & 23680 . \\
\hline$L_{2}^{2}$ & 85.81 & 131.9 & 176.6 & 215.7 & 251.3 & 284.7 & 316.4 \\
\hline$p$ & .01393 & .02396 & .03369 & .04325 & .05158 & .05922 & .06635 \\
\hline \multicolumn{6}{|c|}{ COMPOSITION BY WEIGHT FRACTION: } & \multicolumn{2}{|c|}{ DENSITY VARIATIONS AND REFERENCE } \\
\hline & $\mathrm{Ba} \quad 1.00$ & & & & & $\begin{array}{l}\mathrm{HCP} \\
\mathrm{RH}\end{array}$ & $\begin{array}{l}\mathrm{gm} / \mathrm{cm}^{3} \\
\mathrm{gm} / \mathrm{cm}^{3}\end{array}$ \\
\hline
\end{tabular}

\begin{tabular}{|c|c|c|c|c|c|c|c|}
\hline TEMPERATURE: & $60 \% \mathrm{~F}$ & $500 \mathrm{~F}$ & $1000 \mathrm{~F}$ & $15000 \mathrm{~F}$ & $20000 \mathrm{~F}$ & $2500 \mathrm{~F}$ & $3000{ }^{\circ} \mathrm{F}$ \\
\hline$D_{1}$ & .6873 & .6917 & .6949 & .6962 & .6984 & .7002 & .7018 \\
\hline$D_{2}$ & .4945 & .4964 & .4970 & .4972 & .4972 & .4973 & .4973 \\
\hline$\Sigma_{a_{1}}$ & .0001049 & .00007951 & .00006548 & .00005507 & .00004966 & .00004570 & .00004264 \\
\hline$\Sigma_{a_{2}}$ & .001097 & .00081 .32 & .0006594 & .0005691 & .0005080 & .0004631 & .0004284 \\
\hline$\Sigma_{s_{1}}$ & .006796 & .007027 & .007195 & .007339 & .007427 & .007500 & .007562 \\
\hline$d$ & 1.111 & 1.101 & 1.095 & 1.090 & 1.087 & 1.085 & 1.083 \\
\hline$d_{1}$ & 1.465 & 1.474 & 1.481 & 1.484 & 1.489 & 1.493 & 1.496 \\
\hline$d_{2}$ & 1.054 & 1.058 & 1.059 & 1.060 & 1.060 & 1.060 & 1.060 \\
\hline$L_{1}^{2}$ & 99.60 & 97.33 & 95.72 & 94.15 & 93.40 & 92.80 & 92.28 \\
\hline$L_{2}^{2}$ & 450.8 & 610.4 & 753.8 & 873.7 & 978.8 & 1074. & 1161. \\
\hline$p$ & .9848 & .9888 & .9910 & .9926 & .9934 & .9939 & .9944 \\
\hline
\end{tabular}

COMPOSITION QY WEIGHT FRACTION:

Be 1.00

REFERENCE:
DENSITY YARIATIONS AND REFERENCE: ANPD: E. A. Aitken

$\begin{array}{ll} & 1.85 \mathrm{gm} / \mathrm{cm}^{3} \\ \mathrm{HCP} & 1.84 \mathrm{gm} / \mathrm{cm}^{3} \\ \mathrm{RH} & 1.85 \mathrm{gm} / \mathrm{cm}^{3} \\ \mathrm{MH} & 1.82 \mathrm{gm} / \mathrm{cm}^{3}\end{array}$


$-$

MATERIAL: Beryllium $\left(n_{s}, 2 n\right)$

ASSUMEO DENSITY: $\quad 1.85 \quad \mathrm{gm}^{\mathrm{m}} / \mathrm{cm}^{\mathrm{a}}$

\begin{tabular}{|c|c|c|c|c|c|c|c|}
\hline TEMPERATURE: & $60 \% \mathrm{~F}$ & $5000 \mathrm{~F}$ & $1000 \mathrm{~F}$ & $1500 \% \mathrm{~F}$ & $2000 \mathrm{FF}$ & $2500 \mathrm{FF}$ & $30000 \mathrm{~F}$ \\
\hline$D_{1}$ & .7002 & .7051 & .7087 & .7103 & .7127 & .7147 & .7165 \\
\hline$D_{2}$ & 4945 & .4964 & .4970 & .4972 & .4972 & . 4973 & .4973 \\
\hline$\Sigma_{a_{1}}$ & .001401 & .001414 & .001430 & .001445 & .001455 & .001464 & .001473 \\
\hline$\Sigma_{a_{2}}$ & .001097 & .0008132 & .0006594 & .0005691 & .0005080 & .0004631 & .0004284 \\
\hline$\Sigma_{s,}$ & .006968 & .007211 & .007387 & .007539 & .007632 & .007709 & .007775 \\
\hline$d$ & 1.114 & 1.103. & 1.096 & 1.092 & 1.089 & 1.086 & 1.084 \\
\hline$d_{1}$ & 1.492 & 1.503 & 1.511 & 1.514 & 1.519 & 1.523 & 1.527 \\
\hline$d_{2}$ & 1.054 & 1.058 & 1.059 & 1.060 & 1.060 & 1.060 & 1.060 \\
\hline$L_{1}^{2}$ & 83.67 & 81.74 & 80.38 & 79.06 & 78.43 & 77.91 & 77.48 \\
\hline$L_{\frac{2}{2}}^{2}$ & 450.8 & 610.4 & 753.8 & 873.7 & 978.8 & 1074. & 1161. \\
\hline$p$ & .8326 & .8360 & .8379 & .8392 & .8399 & .8404 & .8407 \\
\hline COMPOSITION QY & IGHT FRAC & & & & & ENSITY VARIATIONS & AND REFERENCE: \\
\hline REFERENCE: & Be 1.00 & & & , & & $\begin{array}{l}\text { : } \mathbf{E}_{\mathrm{s}} \text { A. Aitken } \\
\text { HCP } \\
\text { RH } \\
\text { MH }\end{array}$ & $\begin{array}{l}1.85 \mathrm{gm} / \mathrm{cm}^{3} \\
1.84 \mathrm{gm} / \mathrm{cm}^{3} \\
1.85 \mathrm{gm} / \mathrm{cm}^{3} \\
1.82 \mathrm{gm} / \mathrm{cm}^{3}\end{array}$ \\
\hline
\end{tabular}

material: Beryilium (n, 2n)

\begin{tabular}{|c|c|c|c|c|c|c|c|}
\hline TEMPERATURE & $\infty$ & SoOF & $1000 \mathrm{~F}$ & $15000 \mathrm{~F}$ & $20000 \mathrm{~F}$ & $2500 \mathrm{~F}^{\mathrm{F}}$ & $3000{ }^{\circ} \mathrm{F}$ \\
\hline$v \Sigma_{f_{1}}$ & .0007506 & .0007736 & .0007907 & .0008057 & .0008150 & .0008227 & .0008294 \\
\hline $\boldsymbol{\nu} \Sigma_{f_{2}}$ & 0 & 0 & 0 & 0 & 0 & 0 & 0 \\
\hline$k_{1}$ & .5358 & .5469 & .5531 & .5577 & .5600 & .5618 & .5631 \\
\hline$k_{2}$ & 0 & 0 & 0 & 0 & 0 & 0 & 0 \\
\hline
\end{tabular}




\begin{tabular}{|c|c|c|c|c|c|c|c|}
\hline TEMPERATURE: & $68 \mathrm{~F}$ & $800 \mathrm{~F}$ & $1000 \mathrm{~F}$ & $1500 \% \mathrm{~F}$ & $2000{ }^{\circ} \mathrm{F}$ & $25000 \mathrm{~F}$ & $3000 \mathrm{~F}$ \\
\hline$b_{1}$ & .6445 & .6482 & .6508 & .6520 & .6537 & .6550 & .6562 \\
\hline$D_{2}$ & .4872 & .4983 & .5036 & .5063 & .5078 & .5088 & .5095 \\
\hline$\Sigma_{a_{1}}$ & .00006398 & .00004878 & .00004032 & .00003432 & .00003098 & .00002853 & .00002664 \\
\hline & & & & - & & & \\
\hline$\Sigma_{a_{2}}$ & .0006560 & .0004862 & .0003945 & .0003402 & .0003036 & .0002766 & .0002562 \\
\hline$\Sigma_{s_{1}}$ & .005705 & .005892 & .006030 & .006145 & .006220 & .006282 & .006335 \\
\hline$d$ & 1.073 & 1.087 & 1.093 & 1.095 & 1.097 & .1 .098 & 1.098 \\
\hline$d_{1}$ & 1.374 & 1.382 & 1. 387 & 1.390 & 1. 393 & 1. 396 & 1.399 \\
\hline$d_{2}$ & 1.038 & 1.062 & 1.073 & 1.079 & 1.082 & 1.085 & 1.086 \\
\hline$L_{12}^{2}$ & 111.7 & 109.1 & 107.2 & 105.5 & 104,6 & 103,8 & 103.1 \\
\hline$\quad L \frac{2}{2}$ & 742.6 & 1025. & 1276. & 1488. & 1673. & 1840. & 1989. \\
\hline$p$ & .9889 & .9918 & .9934 & .9944 & .9950 & $\because 9955$ & .9958 \\
\hline
\end{tabular}

COMPOSITION GY WEIGHT FRACTION:

$$
\mathrm{Be} .3603
$$

REFERENCE:

$$
\text { O } .6397
$$

DENSITY VARIATIONS AND REFERENCE: ANPD: E A Aitken HCP
$3.02 \mathrm{gm} / \mathrm{cm}^{3}$

$3.01 \mathrm{gm} / \mathrm{cm}^{3}$

MATERIAL: Bismuth

ASSUMED DENSITY: $9.747 \mathrm{gm} / \mathrm{cm}{ }^{\circ}$

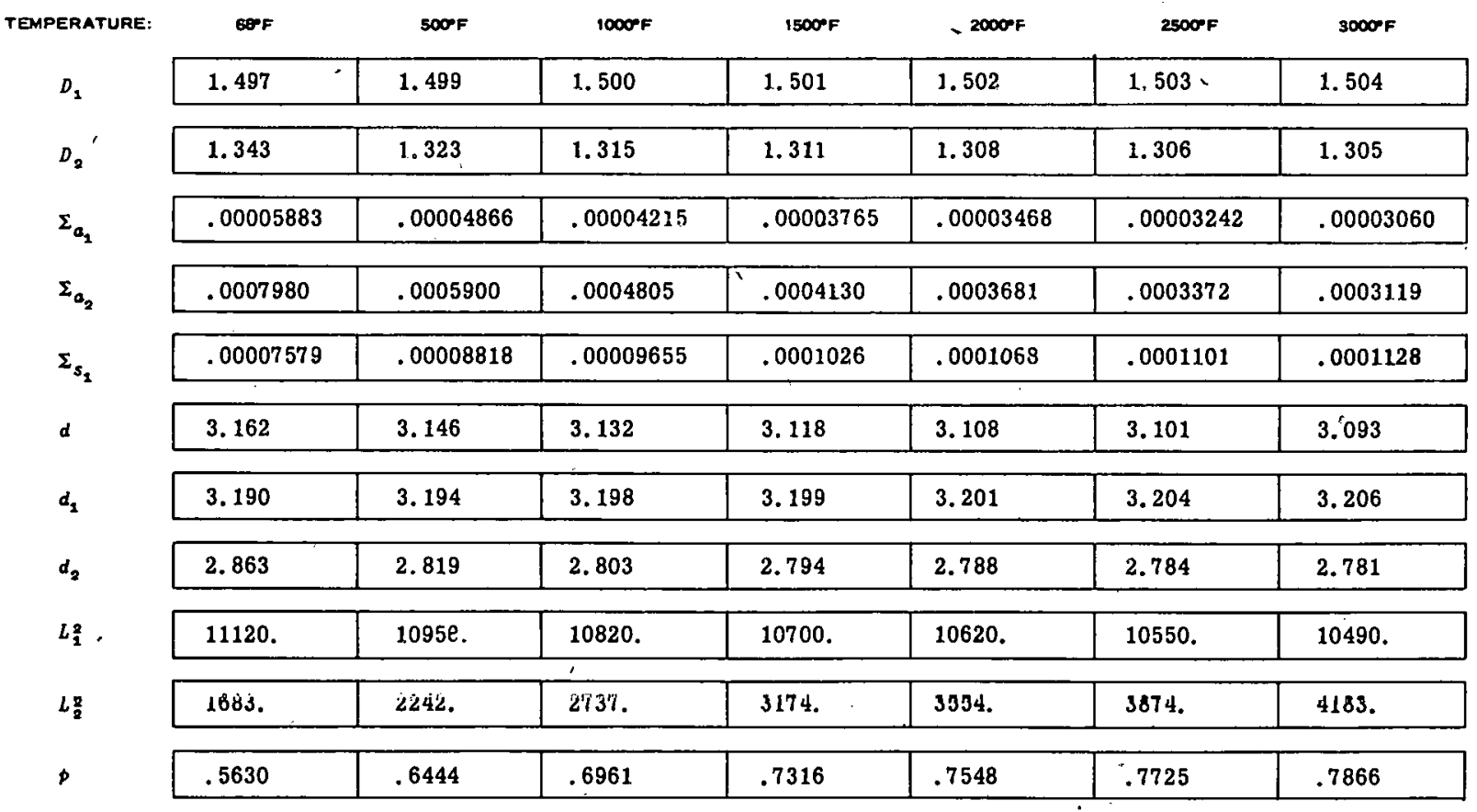

COMPOSITION BY WEIGHT FRACTION:

B1 1.00

REFERENCE:

DONSITY VARIATIONS AND REFEREMCE:

HCP

RH
$9.747 \mathrm{gm} / \mathrm{cm}^{3}$

$9.8 \mathrm{gm} / \mathrm{cm}^{3}$ 


\begin{tabular}{|c|c|c|c|c|c|c|c|}
\hline TEMPERATURE: & $\infty \mathrm{F}$ & $500 \mathrm{~F}$ & $1000 \mathrm{~F}$ & $1500 \mathrm{~F}$ & $2000 \mathrm{~F}$ & $25000 \mathrm{~F}$ & $90000 \mathrm{~F}$ \\
\hline$D_{1}$ & 1.613 & 1.613 & 1.613 & 1.613 & 1.613 & 1.613 & 1.613 \\
\hline$D_{2}$ & .02224 & .02987 & .03670 & .04240 & .04737 & .05184 & .05593 \\
\hline$\Sigma_{a_{1}}$ & .007782 & .007782 & .007782 & .007782 & .007782 & .007782 & .007782 \\
\hline$\Sigma_{a_{2}}$ & 14.82 & 10.99 & 8.910 & 7.690 & 6.864 & 6.257 & 5.787 \\
\hline$\Sigma_{s_{1}}$ & $1.259 \times 10^{-9}$ & $2.549 \times 10^{-9}$ & $3.905 \times 10^{-9}$ & $5.562 \times 10^{-9}$ & $7.152 \times 10^{-9}$ & $8.674 \times 10^{-9}$ & $1.016 \times 10^{-8}$ \\
\hline$d$ & 3.438 & 3.438 & 3.438 & 3.438 & 3.438 & 3.438 & 3.438 \\
\hline$d_{1}$ & 3.438 & 3.438 & 3.438 & 3.438 & 3.438 & 3.438 & 3.438 \\
\hline$d_{2}$ & .04740 & .06366 & .07823 & .09037 & .1010 & .1105 & .1192 \\
\hline$L_{1}^{2}$ & 207.3 & 207.3 & 207.3 & 207.3 & 207.3 & 207.3 & 207.3 \\
\hline$L_{2}^{2}$ & .001501 & .002718 & .004119 & .005513 & .006902 & .008286 & .009664 \\
\hline p & $1.618 \times 10^{-7}$ & $3.276 \times 10^{-7}$ & $5.018 \times 10^{-7}$ & $7.148 \times 10^{-7}$ & $9.190 \times 10^{-7}$ & $1.115 \times 10^{-6}$ & $1.305 \times 10^{-6}$ \\
\hline
\end{tabular}

COMPOSITION GY WEIGHT FRACTION: B .157

C .043

A] .800

ANPD: E. A. Aitken
DENSITY VARIATIONS AND REFERENCE: ANPD: E. A Aitken

$2.53 \mathrm{gm} / \mathrm{cm}^{3}$

\begin{tabular}{|c|c|c|c|c|c|c|c|}
\hline TEMPERATURE: & $\infty F^{\circ}$ & $500 \mathrm{~F}$ & $1000 \mathrm{~F}$ & $15000 \mathrm{~F}$ & $20000 \mathrm{~F}$ & $2500 \mathrm{~F}$ & $3000 \mathrm{~F}$ \\
\hline$D_{1}$ & .6430 & .6430 & .6430 & .6430 & .6430 & .6430 & .6430 \\
\hline$D_{2}$ & .003799 & .005114 & .006296 & .007284 & .008150 & .008930 & .009645 \\
\hline$\Sigma_{a_{1}}$ & .03211 & .03211 & .03211 & .03211 & .03211 & .03211 & .03211 \\
\hline$\Sigma_{a_{2}}$ & 87.24 & 64.69 & 52.46 & 45.27 & 40.41 & 36.84 & 34.07 \\
\hline$\Sigma_{s_{1}}$ & $7.387 \times 10^{-10}$ & $1.497 \times 10^{-9}$ & 2. $295 \times 10^{-9}$ & $3.271 \times 10^{-9}$ & 4. $208 \times 10^{-9}$ & $5.105 \times 10^{-9}$ & $5.980 \times 10^{-9}$ \\
\hline$d$ & 1.371 & 1.371 & 1.371 & 1. 371 & 1.371 & 1.371 & 1.371 \\
\hline$d_{1}$ & 1.371 & 1.371 & 1.371 & 1.371 & 1.371 & 1.371 & 1.371 \\
\hline$d_{2}$ & .008099 & .01090 & .01342 & .01553 & .01737 & .01903 & .02056 \\
\hline$L_{1}^{2}$ & 20.03 & 20.03 & 20.03 & 20.03 & 20.03 & 20.03 & 20.03 \\
\hline$L_{2}^{2}$ & .00004355 & .00007905 & .0001200 & .0001600 & .0002017 & .0002424 & .0002831 \\
\hline$p$ & $2.301 \times 10^{-8}$ & $4.664 \times 10^{-8}$ & $7.147 \times 10^{-8}$ & $1.019 \times 10^{-7}$ & $1.310 \times 10^{-7}$ & $1.580 \times 10^{-7}$ & $1.863 \times 10^{-7}$ \\
\hline
\end{tabular}

COMPOSITION BY WEIGHT FRACTION:

$$
\text { B } 1.00
$$

REFERENCE:
DENSITY VARIATIONS AND REFERENCE:

HCP

ANPD: E. A. Aitken

$$
2.34 \mathrm{gm} / \mathrm{cm}^{3}
$$

$2.45 \mathrm{gm} / \mathrm{cm}^{3}$ 


\begin{tabular}{|c|c|c|c|c|c|c|c|}
\hline TEMPEAATURE. & $\infty \mathrm{F}$ & $500 \mathrm{~F}$ & $10000 \mathrm{r}$ & $1600 \times F$ & mpref & $25 \mathrm{~mm} F$ & $30000 F$ \\
\hline$D_{1}$ & .9510 & .9510 & .9510 & .9510 & .9510 & .9510 & .9510 \\
\hline$D_{2}$ & .0007180 & .0009679 & .001193 & .001382 & .001548 & .001698 & .001835 \\
\hline$\Sigma_{a_{1}}$ & .08164 & .08164 & .08164 & .08164 & .08164 & .08164 & .08164 \\
\hline$\Sigma_{a_{2}}$ & 463.8 & 343.9 & 278.8 & 240.7 & 214.8 & 195.8 & 181.1 \\
\hline$\Sigma_{s_{1}}$ & $1.398 \times 10^{-12}$ & $2.838 \times 10^{-12}$ & $4.352 \times 10^{-12}$ & $6.210 \times 10^{-12}$ & $7.994 \times 10^{-12}$ & $9.703 \times 10^{-12}$ & $1.137 \times 10^{-11}$ \\
\hline$d$ & 2.027 & 2.027 & 2.027 & 2. 027 & 2.027 & 2. 027 & 2.027 \\
\hline$d_{1}$ & 2.027 & 2.027 & 2.027 & 2.027 & 2.027 & 2.027 & 2. 027 \\
\hline$d_{2}$ & .001530 & .002063 & .002544 & .002946 & .003300 & .003619 & .003912 \\
\hline$L_{2}^{2}$ & 11.65 & 11.65 & 11.65 & 11.65 & 11.65 & 11.65 & 11.65 \\
\hline$L_{2}^{2}$ & $1.548 \times 10^{-6}$ & $2.815 \times 10^{-6}$ & $4.280 \times 10^{-6}$ & $5.744 \times 10^{-6}$ & $7.208 \times 10^{-6}$ & $8.671 \times 10^{-6}$ & .00001013 \\
\hline p & $1.713 \times 10^{-11}$ & $3.477 \times 10^{-11}$ & $5.331 \times 10^{-11}$ & $7.607 \times 10^{-11}$ & $9.792 \times 10^{-11}$ & $1.189 \times 10^{-10}$ & $1.393 \times 10^{-10}$ \\
\hline
\end{tabular}

COMPOSITION BY WEIGHT FRACTION:

$$
\mathrm{B}^{10} \quad 1.00
$$

REFERENCE:
OENSITY VARIATIONS AND REFERENCE: HCP $2.168 \mathrm{gm} / \mathrm{cm}^{3}$ (computed)
MAIERIAL: BORON Carbide

ASSIIMEO DENSITY

2. $519 \mathrm{gm} / \mathrm{cm}^{\circ}$

\begin{tabular}{|c|c|c|c|c|c|c|c|}
\hline TEMPERATURE: & $60 \% F$ & $500 \mathrm{~F}$ & $10000^{\circ} \mathrm{F}$ & $1500^{\circ} \mathrm{F}$ & $2000 \mathrm{~F}$ & $2500 \mathrm{~F}$ & $3000^{\circ} \mathrm{F}$ \\
\hline$D_{1}$ & .6445 & .6445 & .6445 & .6445 & .6445 & .6445 & .6445 \\
\hline$D_{2}$ & .004502 & .006055 & .007451 & .008617 & .009638 & .01056 & .01140 \\
\hline$\Sigma_{a_{1}}$ & .02927 & .02927 & .02927 & .02927 & .02927 & .02927 & .02927 \\
\hline$\Sigma_{a_{2}}$ & 73.52 & 54.51 & 44.20 & 38.15 & 34.05 & 31.04 & 28.71 \\
\hline$\Sigma_{s_{1}}$ & $1.731 \times 10^{-9}$ & $3.507 \cdot \times 10^{-9}$ & $5.372 \times 10^{-9}$ & $7.655 \times 10^{-9}$ & $9.845 \times 10^{-9}$ & $1.194 \times 10^{-8}$ & $1.399 \times 10^{-8}$ \\
\hline$d$ & 1.374 & 1.374 & 1.374 & 1.374 & 1.374 & 1.374 & 1.374 \\
\hline$d_{1}$ & 1.374 & 1.374 & 1.374 & j. 374 & 1.374 & 1.374 & 1.374 \\
\hline$d_{2}$ & .009595 & .01291 & .01588 & .01837 & .02054 & .02250 & .02429 \\
\hline$L_{\mathrm{i}}^{2}$ & 22.02 & 22.02 & 22.02 & 22.02 & 22.02 & 22.02 & 22.02 \\
\hline$L \frac{2}{2}$ & .00006123 & .0001111 & .0001686 & .0002259 & .0002830 & .0003401 & .0003969 \\
\hline$p$ & $5.912 \times 10^{-8}$ & $1.198 \times 10^{-7}$ & $1.835 \times 10^{-7}$. & $2.615 \times 10^{-7}$ & $3.363 \times 10^{-7}$ & $4.080 \times 10^{-7}$ & $4.779 \times 10^{-7}$ \\
\hline
\end{tabular}

COMPOSITION BY WEIGHT FRACTION:

$$
\text { B } .7828
$$$$
\text { C } .2172
$$

C .2172

REFERENCE:

(n)




\begin{tabular}{|c|c|c|c|c|c|c|c|}
\hline TEMPERATURE: & $\cos F$ & $5000 \mathrm{~F}$ & $1000 \mathrm{~F}$ & $15000 \mathrm{~F}$ & $2000 \mathrm{~F}$ & $2500 \mathrm{~F}$ & $3000 \mathrm{~F}$ \\
\hline$D_{1}$ & 1.123 & 1.123 & 1.123 & 1.123 & 1.123 & 1.123 & 1.123 \\
\hline$D_{2}$ & .002386 & .001966 & .001859 & .001989 & .002234 & .002553 & .002927 \\
\hline$\Sigma_{a_{2}}$ & .0005683 & .0005678 & .0005674 & .0005669 & .0005663 & .0005658 & .0005653 \\
\hline$\Sigma_{a_{2}}$ & 139.3 & 168.8 & 178.3 & 166.6 & 148.2 & 129.6 & 113.0 \\
\hline$\Sigma_{s_{1}}$ & $4.112 \times 10^{-7}$ & $8.348 \times 10^{-7}$ & $1.280 \times 10^{-6}$ & $1.827 \times 10^{-6}$ & $2.352 \times 10^{-6}$ & $2.855 \times 10^{-6}$ & $3.346 \times 10^{-6}$ \\
\hline$d$ & 2. 395 & 2.395 & 2.395 & 2.395 & 2.395 & 2.395 & 2.395 \\
\hline$d_{1}$ & 2.395 & 2.395 & 2.395 & 2.395 & 2.395 & 2.395 & 2.395 \\
\hline$d_{2}$ & .005085 & .004190 & .003962 & .004239 & .004763 & .005442 & .006239 \\
\hline$L_{1}^{2}$ & 1976. & 1976. & 1976. & 1976. & 1976. & 1976. & 1976. \\
\hline$L_{2}^{2}$ & .00001713 & .00001165 & .00001042 & .00001194 & .00001508 & .00001970 & .00002591 \\
\hline$p$ & .0007231 & .001468 & .002251 & .003213 & .004136 & .005021 & .005884 \\
\hline \multicolumn{6}{|c|}{ COMPOSITION BY WEIGHT FAACTION: } & \multicolumn{2}{|c|}{ DENSITY VARIATIONS AND REFERENCE: } \\
\hline ' & $\mathrm{Cd} 1.00$ & & & & & $\begin{array}{l}\mathrm{RH} \\
\mathrm{HCP}\end{array}$ & $\begin{array}{l}8.648 \mathrm{gm} / \mathrm{cm}^{3} \\
8.648{\mathrm{gm} / \mathrm{cm}^{3}}^{3}\end{array}$ \\
\hline
\end{tabular}

\begin{tabular}{|c|c|c|c|c|c|c|c|}
\hline TEMPERATURE: & $600 \mathrm{~F}$ & $500 \mathrm{~F}$ & $1000 \mathrm{~F}$ & $15000 \mathrm{~F}$ & $2000 \mathrm{FF}$ & $2500 \mathrm{~F}$ & $9000 \mathrm{~F}$ \\
\hline$D_{1}$ & 4.834 & 4.835 & 4.836 & 4.836 & 4.837 & 4.837 & 4.837 \\
\hline$D_{2}$ & 4.295 & 4.426 & 4.501 & 4. 545 & 4. 576 & 4. 599 & 4. 617 \\
\hline$\Sigma_{a_{1}}$ & .0002328 & .0002225 & .0002133 & .0002062 & .0002002 & .0001949 & .0001902 \\
\hline$\Sigma_{a_{2}}$ & .008885 & .006589 & .005342 & .004611 & .004115 & .003752 & .003470 \\
\hline$\Sigma_{s_{1}}$, & .00002062 & .00003132 & .00004089 & .00004847 & .00005492 & .00006056 & .00006559 \\
\hline$d$ & 10.30 & 10.30 & 10.30 & 10.30 & 10.30 & 10.30 & 10.30 \\
\hline$d_{2}$ & 10.30 & 10.30 & 10.31 & 10.31 & 10.31 & 10.31 & 10.31 \\
\hline$d_{g}$ & 9.155 & 9.434 & 9.593 & 9.689 & 9.754 & 9.803 & 9.842 \\
\hline$L \frac{2}{1}$ & 19080. & 19050. & 19020. & 18990. & 18960. & 18930. & 18910. \\
\hline$L_{2}^{2}$ & 483.4 & 671.8 & 842.4 & 985.9 & 1112. & 1226. & 1331. \\
\hline$p$ & .08137 & .1234 & .1608 & .1903 & .2153 & .2371 & .2564 \\
\hline
\end{tabular}

COMPOSITION BY WEIGHT FRACTION:

$$
\text { Ca } 1.00
$$

REFERENCE:
DENSITY VARIATIONS AND REFERENCE:

$\begin{array}{ll}\mathrm{HCP} & 1.55 \mathrm{gm} / \mathrm{cm}^{3} \\ \mathrm{RH} & 1.55 \mathrm{gm} / \mathrm{cm}^{3}\end{array}$




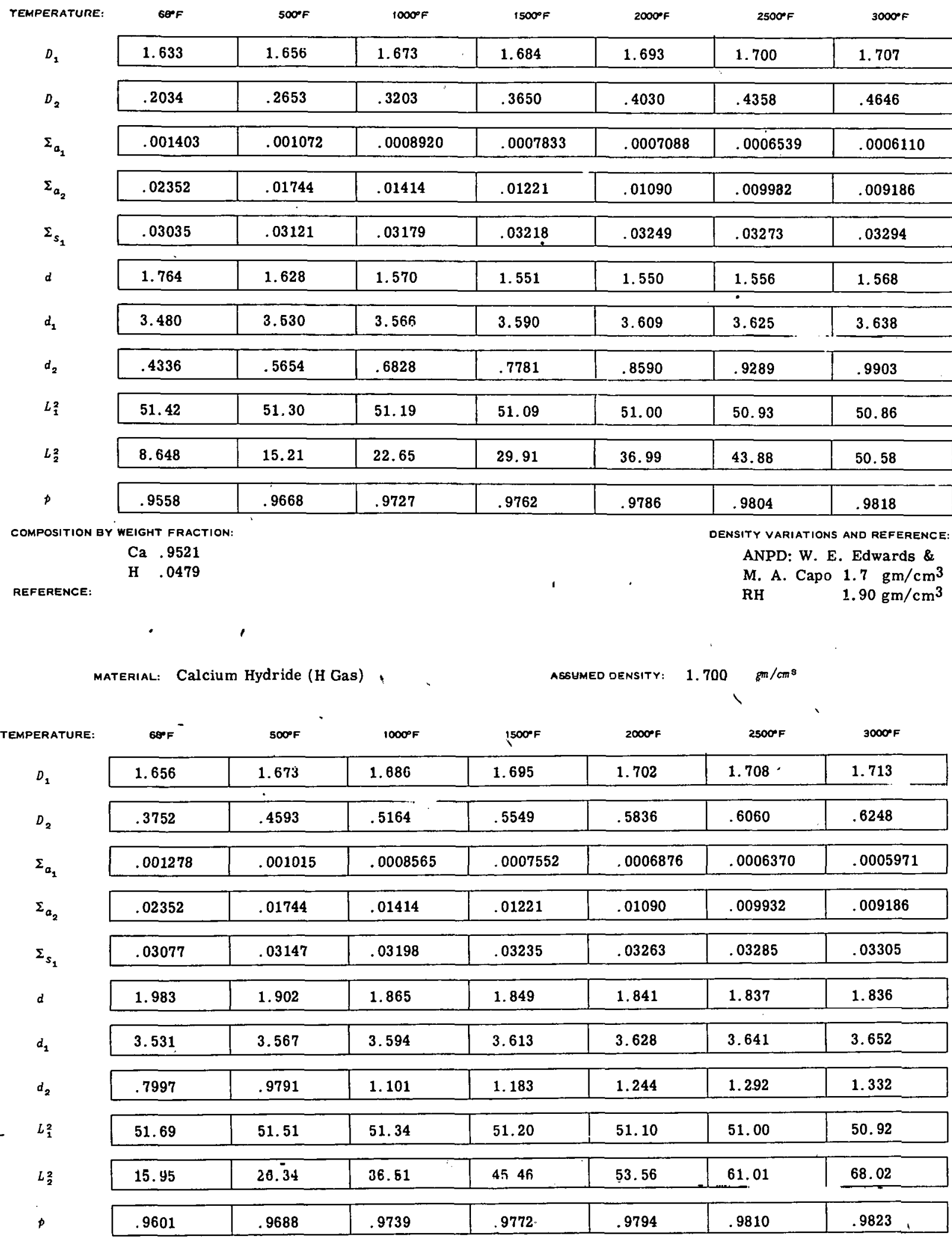

COMPOSITION BY WEIGHT FRACTION:

Ca .9521

H $\quad .0479$

REFERENCE:

(1)

DENSITY VARIATIONS AND REFERENCE: ANPD: W. E. Edwards \& M. A. Capo $1.7 \mathrm{gm} / \mathrm{cm}^{3}$ RH $\quad 1.90 \mathrm{gm} / \mathrm{cm}^{3}$ 


\begin{tabular}{|c|c|c|c|c|c|c|c|}
\hline TEMPERATURE: & . $680 \mathrm{~F}$ & $500 \% \mathrm{~F}$ & $1000^{\circ} \mathrm{F}$ & $1500^{\circ} \mathrm{F}$ & $2000 \% \mathrm{~F}$ & $2500 \% \mathrm{~F}$. & $3000 \mathrm{~F}$ \\
\hline$D_{1}$ & 1.656 & 1.673 & 1.686 & 1.695 & 1.702 & 1.708 & 1.713 \\
\hline$D_{2}$ & .3752 & .4593 & .5164 & .5549 & .5836 & .6060 & .6248 \\
\hline$\Sigma_{a_{1}}$ & .001278 & .001015 & .0008565 & .0007552 & .0006876 & .0006370 & .0005971 \\
\hline$\Sigma_{a_{2}}$ & .02352 & .01744 & .01414 & .01221 & .01090 & .009932 & .009186 \\
\hline$\Sigma_{s,}$ & .03077 & .03147 & .03198 & .03235 & .03263 & .03285 & .03305 \\
\hline$d$ & 1.983 & 1.902 & 1.865 & 1.849 & 1.841 & 1.837 & 1.836 \\
\hline$d_{1}$ & 3.531 & 3.567 & 3.594 & 3.613 & 3628 & 3641 & 3652 \\
\hline$d_{2}$. & .7997 & .9791 & 1.101 & 1. 183 & 1.244 & 1.292 & 1.332 \\
\hline & & & & & & $=$ & \\
\hline$L_{1}^{2}$ & 51.69 & 51.51 & 51.34 & 51.20 & 51.10 & 51.00 & 50.92 \\
\hline$L_{2}^{2}$ & 15.95 & 26.34 & 36.51 & 45.46 & 53.56 & 61.01 & 68.02 \\
\hline$p$ & .9601 & .9688 & .9739 & .9772 & .9794 & .9810 & .9823 \\
\hline
\end{tabular}

COMPOSITION QY WEIGHT FRACTION:

$$
\begin{array}{ll}
\mathrm{Ca} & .9521 \\
\mathrm{H} & .0479
\end{array}
$$

REFERENCE:
ASSUMED DENSITY: $8.960 \quad \mathrm{gm} / \mathrm{cm}$ s ANPD: W. E. Edwards \& M. A. Capo $1.7 \mathrm{gm} / \mathrm{cm}^{3}$ $\mathrm{RH} \quad 1.90 \mathrm{gm} / \mathrm{cm}^{3}$

\begin{tabular}{|c|c|c|c|c|c|c|c|}
\hline TEMPERATURE: & $60 \%$ & $500 \mathrm{~F}$ & $1000 \mathrm{~F}$ & $1500 \mathrm{~F}$ & $20000 \mathrm{~F}$ & $2500 \mathrm{~F}$ & $3000 \mathrm{~F}$ \\
\hline$D_{1}$ & .7729 & .7729 & .7730 & .7730 & .7730 & .7730 & .7730 \\
\hline$D_{2}$ & .3852 & .4065 & .4208 & .4304 & .4374 & .4428 & .4472 \\
\hline$\Sigma_{a_{1}}$ & .001546 & .001541 & .001535 & .001529 & .001524 & .001519 & .001514 \\
\hline$\Sigma_{a_{2}}$ & .2781 & .2062 & .1672 & .1443 & .1288 & .1174 & .1086 \\
\hline$\Sigma_{s_{1}}$ & $6.568 \times 10^{-6}$ & .00001220 & .00001791 & .00002383 & .00002933 & .00003448 & .00003938 \\
\hline$d$ & 1.647 & 1.647 & 1.647 & 1.647 & 1.647 & 1.647 & 1.647 \\
\hline$d_{1}$ & 1.647 & 1.648 & 1.648 & 1.648 & 1.648 & 1.648 & 1.648 \\
\hline$d_{2}$ & .8211 & .8664 & .8970 & .9175 & .9323 & .9439 & .9531 \\
\hline$L_{1}^{2}$ & 497.7 & 497.7 & 497.7 & 497.7 & 497.6 & 497.6 & 497.6 \\
\hline$L \frac{2}{2}$ & 1.3835 & 1.971 & 2.517 & 2.984 & 3.396 & 3.772 & 4.118 \\
\hline$p$ & .004229 & .007858 & .01153 & .01534 & .01888 & .02220 & .02535 \\
\hline
\end{tabular}

MATERIAL: COpper
COMPOSITION GY WEIGHT FRACTION:

Cu 1.00

REFERENCE:
DENSITY VARIATIONS AND REFERENCE:

$\begin{array}{ll}\text { MH } & 8.96 \mathrm{gm} / \mathrm{cm}^{3} \\ \text { HCP } & 8.93-8.95 \mathrm{gm} / \mathrm{cm}^{3} \\ \text { RH } & 8.96 \mathrm{gm} / \mathrm{cm}^{3}\end{array}$




\begin{tabular}{|c|c|c|c|c|c|c|c|}
\hline TEMPERATURE: & $\cos F$ & $800 \mathrm{~F}$ & tooos $\mathrm{F}$ & $15000 \mathrm{~F}$ & $2000 \mathrm{~F}$ & $2500 \mathrm{~F}$ & $3000 \mathrm{~F}$ \\
\hline$D_{2}$ & 1.045 & 1.045 & 1.045 & 1.045 & 1.045 & 1.045 & 1.045 \\
\hline$D_{2}$ & .01050 & 01460 & .01855 & .02202 & .02516 & .02805 & .03071 \\
\hline$\Sigma_{a_{1}}$ & .002991 & .002991 & .002991 & .002991 & .002991 & .002991 & .002991 \\
\hline$\Sigma$ & 28.85 & 20.20 & 15,53 & 12.84 & 1105 & 9763 & 8.805 \\
\hline $\mathbf{\Sigma}_{s_{1}}$ & $2.082 \times 10^{-13}$ & $4.219 \times 10^{-13}$ & $6.464 \times 10^{-13}$ & $9.207 \times 10^{-13}$ & $1.184 \times 10^{-12}$ & $1.436 \times 10^{-12}$ & $1.682 \times 10^{-12}$ \\
\hline$d$ & 2.227 & 2.227 & 2.227 & 2.227 & - 2.227 & 2.227 & 2.227 \\
\hline$d_{1}$ & 2.227 & 2,227 & 2.227 & 2.227 & 2,227 & 2.227 & 2.227 \\
\hline$d_{2}$ & .02238 & . . .03112 & .03954 & .04693 & .05363 & .05979 & .06546 \\
\hline$L_{1}^{2}$ & 349.2 & 349.2 & 349.2 & 349.2 & 349.2 & 349.2 & 349.2 \\
\hline$L_{\frac{2}{2}}^{2}$ & .0003639 & .0007227 & .001194 & .001715 & .002278 & .002873 & .003488 \\
\hline & & & & & & 1 & \\
\hline$p$ & $6.962 \times 10^{-11}$ & $1.410 \times 10^{-10}$ & $2.161 \times 10^{-10}$ & $3.078 \times 10^{-10}$ & $3.958 \times 10^{-10}$ & $4.802 \times 10^{-10}$ & $5.624 \times 10^{-10}$ \\
\hline MPOSITION BY & ZIGHT FRACTION: & & & & & IENSITY VARIATION & AND REFERENCE: \\
\hline & Dy 1.00 & & & & & $\mathrm{RMH}$ & $8.562 \mathrm{gm} / \mathrm{cm}^{3}$ \\
\hline
\end{tabular}

REFERENCE:

\begin{tabular}{|c|c|c|c|c|c|c|c|}
\hline TEMPERATURE: & $60 \mathrm{~F}$ & $500 \mathrm{~F}$ & $1000 \mathrm{PF}$ & $1500^{\circ} \mathrm{F}$ & $20000 \mathrm{~F}$ & $2500 \mathrm{~F}$ & $3000 \mathrm{~F}$ \\
\hline$D_{1}$ & 1.634 & 1.634 & 1.634 & 1.634 & 1.634 & 1.634 & 1.634 \\
\hline$D_{2}$ & .004264 & .006764 & .007980 & .007582 & .006919 & .006473 & .006253 \\
\hline$\Sigma_{a_{1}}$ & .02563 & .02563 & .02563 & .02563 & .02563 & .02563 & .02563 \\
\hline$\Sigma_{a_{2}}$ & 77.95 & 49.08 & 41.53 & 43.63 & 47.77 & 51.03 & 52.79 \\
\hline$\Sigma_{s_{1}}$ & $7.374 \times 10^{-20}$ & 1. $497 \times 10^{-19}$ & $2.295 \times 10^{-19}$ & $3.276 \times 10^{-19}$ & 4. $218 \times 10^{-19}$ & 5. $120 \times 10^{-19}$ & $6.000 \times 10^{-19}$ \\
\hline$d$ & 3.483 & 3.483 & 3.483 & 3.483 & 3.483 & 3.483 & 3.483 \\
\hline$d_{1}$ & 3.483 & 3.483 & 3.483 & 3.483 & 3.483 & 3.483 & 3.483 \\
\hline$d_{2}$ & .009089 & .01442 & .01701 & .01616 & .01475 & .01380 & .01333 \\
\hline$L_{1}^{2}$ & 63.75 & 63.75 & 63.75 & 63.75 & 63.75 & 63.75 & 63.75 \\
\hline$L \frac{2}{2}$ & .00005470 & .0001378 & .0001922 & .0001738 & .0001448 & .0001268 & .0001185 \\
\hline$p$ & $2.877 \times 10^{-18}$ & $5.840 \times 10^{-18}$ & $8.955 \times 10^{-18}$ & $1.278 \times 10^{-17}$ & $1.645 \times 10^{-17}$ & $1.997 \times 10^{-17}$ & $2.341 \times 10^{-17}$ \\
\hline
\end{tabular}

COMPOSITION BY WEIGHT FRACTION:

Eu 1.00
DENSITY VARIATIONS AND REFERENCE: ANPD: E. A. Aitken $5.166 \mathrm{gm} / \mathrm{cm}^{3}$

REFERENCE: 


\begin{tabular}{|c|c|c|c|c|c|c|c|}
\hline EMPERATURE: & $60^{\circ} \mathrm{F}$ & $5000 \mathrm{~F}$ & $1000 \mathrm{~F}$ & $1500 \mathrm{~F}$ & $2000 \% \mathrm{~F}$ & $2500^{\circ} \mathrm{F}$ & $3000 \mathrm{~F}$ \\
\hline$D_{1}$ & .9638 & .9638 & .9638 & .9638 & .9638 & .9638 & .9638 \\
\hline$D_{2}$ & .003323 & .005267 & .006212 & .005903 & .005388 & .005041 & .004871 \\
\hline$\Sigma_{a_{1}}$ & .03603 & .03603 & .03603 & .03603 & .03603 & .03603 & .03603 \\
\hline$\Sigma_{a_{2}}$ & 99.88 & 62.88 & 53.20 & 55.91 & 61.21 & 65.38 & 67.63 \\
\hline$\Sigma_{s_{1}}$ & $3.344 \times 10^{-15}$ & $6.784 \times 10^{-15}$ & $1.040 \times 10^{-14}$ & $1.483 \times 10^{-14}$ & $1.909 \times 10^{-14}$ & $2.318 \times 10^{-14}$ & $2.716 \times 10^{-14}$ \\
\hline$d$ & 2. 054 & 2.054 & 2.054 & 2.054 & 2.054 & 2.054 & 2.054 \\
\hline$d_{1}$ & 2.054 & 2.054 & 2.054 & 2.054 & 2.054 & 2.054 & 2.054 \\
\hline$d_{2}$ & .007083 & .01123 & .01324 & .01258 & .01148 & .01075 & .01038 \\
\hline$L L_{1}^{2}$ & 26.75 & 26.75 & 26.75 & 26.75 & 26.75 & 26.75 & 26.75 \\
\hline$L \frac{2}{2}$ & .00003327 & .00008376 & .0001167 & .0001056 & .00008803 & .00007711 & .00007202 \\
\hline$p$ & $9.284 \times 10^{-14}$ & $1.883 \times 10^{-13}$ & $2.887 \times 10^{-13}$ & $4.118 \times 10^{-13}$ & $5.300 \times 10^{-13}$ & $6.433 \times 10^{-13}$ & $7.539 \times 10^{-13}$ \\
\hline
\end{tabular}

COMPOSITION GY WEIGHT FRACTION:

$$
\begin{array}{ll}
\text { Eu } & .8636 \\
\text { O } & .1364
\end{array}
$$

REFERENCE:
DENSITY VARIATIONS AND REFERENCE:

HCP $7.42 \mathrm{gm} / \mathrm{cm}^{3}$
MATERIAL: Gadolinium

\begin{tabular}{|c|c|c|c|c|c|c|c|}
\hline TEMPERATURE: & $600 \mathrm{~F}$ & $500 \mathrm{FF}$ & $1000 \mathrm{~F}$ & $1500^{\circ} \mathrm{F}$ & $2000 \mathrm{~F}$ & $2500 \% \mathrm{~F}$ & $3000 \mathrm{~F}$ \\
\hline$D_{1}$ & 1.944 & 1.944 & 1.944 & 1.944 & 1.944 & 1.944 & 1.944 \\
\hline $\mathrm{D}_{2}$ & .0003057 & .0004863 & .0007319 & .001019 & .001346 & .001714 & .002122 \\
\hline$\Sigma_{a_{1}}$ & .003435 & .003435 & .003435 & .003435 & .003435 & .003435 & .003435 \\
\hline$\Sigma_{a_{2}}$ & 1086. & 681.3 & 451.9 & 324.2 & 244.9 & 192.1 & 155.0 \\
\hline$\Sigma_{s_{1}}$ & $1.122 \times 10^{-15}$ & $2.279 \times 10^{-15}$ & $3.494 \times 10^{-15}$ & $4.986 \times 10^{-15}$ & $6.419 \times 10^{-15}$ & $7.791 \times 10^{-15}$ & $9.131 \times 10^{-15}$ \\
\hline$d$ & 4.144 & 4.144 & 4.144 & 4.144 & 4. 144 & 4.144 & 4.144 \\
\hline$d_{1}$ & 4.144 & 4.144 & 4.144 & 4.144 & 4.144 & 4.144 & 4. 144 \\
\hline$d_{2}$ & .0006515 & .001037 & .001560 & .002171 & $.00287 \theta$ & .003654 & .004523 \\
\hline$L_{1}^{2}$ & 566.1 & 566.1 & 566.1 & 566.1 & 566.1 & 566.1 & 566.1 \\
\hline * $\quad L_{2}^{2}$ & $2.815 \times 10^{-7}$ & $7.137 \times 10^{-7}$ & $1.620 \times 10^{-6}$ & $3.142 \times 10^{-6}$ & $5.498 \times 10^{-6}$ & $8.926 \times 10^{-6}$ & $1.369 \times 10^{-5}$ \\
\hline$p$ & $3.268 \times 10^{-13}$ & $6.634 \times 10^{-13}$ & $1.017 \times 10^{-12}$ & $1.452 \times 10^{-12}$ & $1.869 \times 10^{-12}$ & $2.269 \times 10^{-12}$ & $2.659 \times 10^{-12}$ \\
\hline
\end{tabular}

ASSUMEO DENSITY: $\quad 7.868 \mathrm{gm} / \mathrm{cm}^{2}$
COMPOSITION BY WEIGHT FRACTION:

Gd 1.00
DENSITY VARIATIONS AND REFERENCE: ANPD: E. A. Aitken

$7.868 \mathrm{gm} / \mathrm{cm}^{3}$

REFERENCE: 


\begin{tabular}{|c|c|c|c|c|c|c|c|}
\hline TEMPERATURE: & $60 \% \mathrm{~F}$ & $500 \mathrm{~F}$ & $1000^{\circ} \mathrm{F}$ & $1500^{\circ} \mathrm{F}$ & $2000 \mathrm{~F}$ & $2500 \mathrm{~F}$ & $3000 \% 5$ \\
\hline$D_{1}$ & 1.404 & 1.404 & 1.404 & 1.404 & 1.404 & 1.404 & 1.404 \\
\hline$D_{2}$ & .0003741 & .0005952 & .0008957 & .001246 & .001647 & .002097 & .002595 \\
\hline $\bar{\Sigma}_{a_{1}}$ & .005348 & .005348 & .005348 & .005348 & $.005348^{\prime}$ & .005348 & .005348 \\
\hline$\Sigma_{a_{2}}$ & 887.0 & 556.5 & 369.1 & 264.8 & 200.0 & 156.9 & 126.6 \\
\hline$\Sigma_{s_{1}}$ & $1.779 \times 10^{-10}$ & $3.611 \times 10^{-10}$ & $5.538 \times 10^{-10}$ & $7.900 \times 10^{-10}$ & $1.017 \times 10^{-9}$ & $1.234 \times 10^{-9}$ & $1.446 \times 10^{-9}$ \\
\hline$d$ & 2.993 & 2.993 & 2.993 & 2.993 & 2.993 & 2.993 & 2.993 \\
\hline$d_{1}$ & 2.993 & 2.993 & 2.993 & 2.993 & 2.993 & 2.993 & 2.993 \\
\hline$d_{2}$ & .0007975 & .001269 & .001909 & .002657 & .003511 & .004470 & .005531 \\
\hline$L_{1}^{2}$ & 262.6 & 262.6 & 262.6 & 262.6 & 262.6 & 262.6 & $\because 262.6$ \\
\hline$L_{2}^{2}$ & $4.218 \times 10^{-7}$ & $1.069 \times 10^{-6}$ & $2.426 \times 10^{-6}$ & $4.707 \times 10^{-6}$ & $8.234 \times 10^{-6}$ & $1.337 \times 10^{-5}$ & $2.050 \times 10^{-5}$ \\
\hline$p$ & $3.327 \times 10^{-8}$ & $6.753 \times 10^{-8}$ & $1.035 \times 10^{-7}$ & $1.477 \times 10^{-7}$ & $1: 901 \times 10^{-7}$ & $2.308 \times 10^{-7}$ & $2.704 \times 10^{-7}$ \\
\hline
\end{tabular}

COMPOSITION BY WEIGHT FRACTION:

\begin{tabular}{|c|c|c|c|c|c|c|c|}
\hline TEMPERATURE: & $68^{\circ} \mathrm{F}$ & $500 \mathrm{~F}$ & ${ }^{1000 F^{\prime}}$ & $1500^{\circ} \mathrm{F}$ & $2000^{\circ} \mathrm{F}$ & $2500 \% \mathrm{~F}$ & $3000 \mathrm{~F}$ \\
\hline$D_{\mu}$ & .9116 & .9116 & .9116 & .9116 & 9116 & .9116 & .9116 \\
\hline$D_{2}$ & .06060 & .07906 & .09424 & .1058 & .1151 & .1227 & .1291 \\
\hline$\cdot \Sigma_{a_{1}}$ & .006615 & .006615 & .006615 & $.0066: 5$ & .006615 & .006615 & .006615 \\
\hline$\Sigma_{a_{2}}$ & 5.126 & 3.803 & 3,093 & 2,687 & 2.423 & 2.237 & 2.099 \\
\hline$\Sigma_{s_{1}}$ & $1.871 \times 10^{-14}$ & $3.794 \times 10^{-14}$ & $5.815 \times 10^{-14}$ & $8,287 \times 10^{-14}$ & $1.066 \times 10^{-13}$ & $1.293 \times 10^{-13}$ & $1.515 \times 10^{-1.3}$ \\
\hline$d$ & 1.943 & 1.943 & 1.943 & . 1.943 & 1,943 & 1.943 & 1.943 \\
\hline$d_{1}$ & 1.943 & 1.943 & 1.943 & 1.943 & 1.943 & 1.943 & 1.943 \\
\hline$d_{2}$ & .1292 & .1685 & .2009 & .2255 & .2453 & .261 .6 & .2753 \\
\hline$L_{1}^{2}$ & 137.8 & 137.8 & 137.8 & 137.8 & 137.8 & 137,8 & 137.8 \\
\hline$L_{2}^{2}$ & .01182 & .02079 & .03047 & .03938 & .04750 & .05486 & .06152 \\
\hline$p$ & $2.828 \times 10^{-12}$ & $5.735 \times 10^{-12}$ & b $789 \times 10^{-12}$ & $1.253 \times 10^{-11}$ & $1.611 \times 10^{-11}$ & $1.955 \times 10^{-11}$ & $2.290 \times 10^{-11}$ \\
\hline
\end{tabular}




\begin{tabular}{|c|c|c|c|c|c|c|c|}
\hline TEMPERATURE: & $60 \% \mathrm{~F}$. & $500 \mathrm{~F}^{\mathrm{F}}$ & $10000 \mathrm{~F}$ & $1500^{\circ} \mathrm{F}$ & $2000 \mathrm{~F}$ & $2500 \mathrm{~F}$ & $3000 \mathrm{~F}$ \\
\hline$D_{1}$ & 1.192 & 1.199 & 1.205 & 1.209 & 1.213 & 1.215 & 1.218 \\
\hline$D_{2}$ & .9404 & .9294 & .9240 & .9211 & .9193 & .9182 & .9175 \\
\hline$\Sigma_{a_{1}}$ & .00002147 & .00001620 & .00001340 & .00001173 & .00001059 & $9.761 \times 10^{-6}$ & $9.120 \times 10^{-6}$ \\
\hline$\Sigma_{a_{2}}$ & .0002279 & .0001685 & .0001372 & .0001179 & .0001051 & .00009628 & .00008906 \\
\hline$\stackrel{1}{s}_{s_{1}}$ & .002821 & .002908 & .002971 & .003016 & .003051 & .003081 & $.003106^{\circ}$ \\
\hline$d$ & 2.044 & 2.012 & 1.996 & 1.986 & 1.980 & 1.976 & 1.973 \\
\hline$d_{1}$ & 2.541 & 2.557 & 2.569 & 2.578 & 2.585 & 2.591 & 2.596 \\
\hline$d_{2}$ & 2.004 & 1.981 & 1.970 & 1.963 & 1.960 & 1.957 & 1.956 \\
\hline$L_{1}^{2}$ & 419.3 & 410.1 & 403.8 & 399.5 & 396.0 & 393.3 & 390.9 \\
\hline$L_{2}^{2}$ & 4127. & 5516. & 6735. & 7810. & 8747. & 9537. & 10300. \\
\hline$p$ & .9924 & .9945 & .9955 & .9961 & .9965 & .9968 & .9971 \\
\hline
\end{tabular}

COMPOSITION BY WEIGHT FRACTION:

REFERENCE:

$$
\text { C } 1.00
$$

DENSITY VARIATIONS AND REFERENCE:

ANPD: E. A. Aitken

1. 55-1. $75 \mathrm{gm} / \mathrm{cm}^{3}$

MATERIAL: Hafnium

ASSUMED DENSITY: $\quad 13.09 \mathrm{gm}^{\mathrm{c}} / \mathrm{cm}^{\mathrm{s}}$,

\begin{tabular}{|c|c|c|c|c|c|c|c|}
\hline TEMPERATURE: & $685 \mathrm{~F}$ & $500^{\circ} \mathrm{F}$ & $1000^{\circ} \mathrm{F}$ & $1500^{\circ} \mathrm{F}$ & $2000 \mathrm{~F}$ & $2500 \mathrm{~F}$ & $3000^{\circ} \mathrm{F}$ \\
\hline$D_{1}$ & 1.065 & 1.065 & 1.065 & 1.065 & 1.065 & 1.065 & 1.065 \\
\hline$D_{2}$ & .07362 & .09391 & .1086 & .1154 & .1086 & .08713 & .06328 \\
\hline$\Sigma_{a_{1}}$ & .004913 & .004913 & .004913 & .004913 & .004913 & .004913 & .004913 \\
\hline$\Sigma_{a_{2}}$ & 4.176 & 3.198 & 2.717 & 2.531 & 2.698 & 3.424 & 4.818 \\
\hline$\Sigma_{s_{1}}$ & $4.451 \times 10^{-14}$ & $9.024 \times 10^{-14}$ & $1.383 \times 10^{-13}$ & $1.972 \times 10^{-13}$ & $2.537 \times 10^{-13}$ & $3.078 \times 10^{-13}$ & $3.606 \times 10^{-13}$ \\
\hline$d$ & 2.270 & 2.270 & 2.270 & 2.270 & 2.270 & 2.270 & 2.270 \\
\hline$d_{1}$ & 2.270 & 2.270 & 2.270 & 2.270 & 2.270 & 2.270 & 2.270 \\
\hline$d_{2}$ & .1569 & .2002 & .2315 & .2460 & .2315 & .1857 & .1349 \\
\hline$L_{1}^{2}$ & 216.8 & 216.8 & 216.8 & 216.8 & 216.8 & 216.8 & 216.8 \\
\hline$L \frac{2}{2}$ & .01763 & .02937 & .03998 & .04559 & .04026 & .02544 & .01313 \\
\hline$p$ & $9.060 \times 10^{-12}$ & $1.837 \times 10^{-11}$ & $2,815 \times 10^{-11}$ & $4.013 \times 10^{-11}$ & $5.163 \times 10^{-11}$ & $6.265 \times 10^{-11}$ & $7.340 \times 10^{-11}$ \\
\hline
\end{tabular}

COMPOSITION GY WEIGHT FRACTION:

Hf 1.00

OENSITY VARIATIONS AND REFERENCE:

RMH

$13.09 \mathrm{gm} / \mathrm{cm}^{3}$

$11.4 \mathrm{gm} / \mathrm{cm}^{3}$

J. L. Everhart, Bureau of Standards Circular (C447), 1943 


\begin{tabular}{|c|c|c|c|c|c|c|c|}
\hline TEMPERATURE: & $\cos$ & $500 \mathrm{~F}$ & $1000 \% \mathrm{~F}$ & $1500^{\circ} \mathrm{F}$ & $2000 \mathrm{FF}$ & $2500 \mathrm{~F}$ & $3000 \mathrm{~F}$ \\
\hline$D_{1}$ & 1.161 & 1.176 & 1.186 & 1.192 & 1.198 & 1.202 & 1.205 \\
\hline$D_{2}$ & .2239 & .2852 & .3374 & .3782 & .4118 & .4402 & .4646 \\
\hline$\Sigma_{a_{1}}$ & .002445 & .001886 & .001577 & .001389 & .001259 & .001163 & .001088 \\
\hline$\Sigma_{a_{2}}$ & .04245 & .03147 & .02552 & $\cdot .02203$ & .01966 & .01792 & .01658 \\
\hline$\Sigma_{s}$ & .02384 & .02482 & .02544 & .02585 & .02617 & .02642 & .02662 \\
\hline$d$ & 1.757 & 1.669 & 1.625 & 1.605 & 1.596 & 1.595 & 1.596 \\
\hline$d_{1}$ & 2.475 & 2.506 & 2.527 & 2.542 & 2.553 & 2.562 & 2.569 \\
\hline$d_{2}$ & .4772 & .6079 & .7191 & .8062 & .8778 & .9383 & .9903 \\
\hline$L_{1}^{2}$ & 44.17 & 44.02 & 43.88 & 43.77 & 43.67 & 43.58 & 43.50 \\
\hline$L \frac{2}{2}$ & 5.274 & 9.061 & 13.22 & 17. 17 & 20.95 & 24.56 & 28.03 \\
\hline$p$ & .9070 & .9294 & .9416 & .9490 & .9541 & .9578 & .9607 \\
\hline
\end{tabular}

COMPOSITION BY WEIGHT FRACTION:

REFERENCE:

$$
\mathrm{Y} .9845
$$$$
\text { H } .0155\left(\mathrm{~N}_{\mathrm{H}}=4 \times 10^{22}\right)
$$

ANPD: C. R. Simmons
DENSITY VARIATIONS AND REFERENCE ANPD: C. R: Simmons, 1957

$4.33 \mathrm{gm} / \mathrm{cm}^{3}$ ANPD- E. A. Aitken, 1956 $4.45 \mathrm{gm} / \mathrm{cm}^{3}$

mateaIAL, Hydrided Yhtrilum (H Gas)

ACCUMED DEREITY, $4.33 \mathrm{sm} / \mathrm{om}$ "

\begin{tabular}{|c|c|c|c|c|c|c|c|}
\hline TEMPERATURE: & 680 & $500 \mathrm{~F}$ & $1000 \mathrm{~F}$ & $1500 \% \mathrm{~F}$ & $20000 \mathrm{~F}$ & $2500^{\circ} \mathrm{F}$ & $3000 \mathrm{~F}$ \\
\hline$D_{1}$ & 1.178 & 1.187 & 1.195 & 1.200 & 1.204 & 1.207 & 1.210 \\
\hline$D_{2}$ & .3823 & .4553 & .5027 & .5339 & .5566 & .5742 & .5888 \\
\hline$\Sigma_{a}$ & .002238 & .001789 & .001516 & .001340 & .001222 & .001134 & .001064 \\
\hline$\Sigma_{a_{2}}$ & .04244 & .03147 & .02552 & .02203 & .01966 & .01792 & 01657 \\
\hline$\Sigma_{s_{1}}$ & .02427 & .02506 & .02561 & .02600 & .02629 & .02652 & .02671 \\
\hline$d$ & 1.893 & 1.839 & 1.808 & 1.789 & 1.777 & 1.768 & 1.762 \\
\hline$d_{1}$ & 2.510 & 2.531 & 2.546 & 2.557 & 2.566 & 2.573 & 2.579 \\
\hline$d_{2}$ & .8149 & .9704 & 1.072 & 1. 138 & 1.186 & 1.224. & 1.255 \\
\hline$L_{1}^{2}$ & 44.43 & 44.22 & 44.03 & 43.88 & 43.76 & 43.65 & 43.56 \\
\hline$L L_{n}^{2}$ & 9.007 & 14,47 & 19,70 & 24.24 & 28,32 & 32.04 & 35.52 \\
\hline p & .9155 & .9334 & .9441 & .9510 & .9556 & .9590 & .9617 \\
\hline
\end{tabular}

COMPOSITION BY WEIGHT FRACTION:

REFERENCE
$\mathrm{Y} .9845$

H. $0155\left(\mathrm{~N}_{\mathrm{H}}=4 \times 10^{22}\right)$
DENSITY VARIATIONS AND REFERENCE: ANPD: C. R. Simmons, 1957 $4.33 \mathrm{gm} / \mathrm{cm}^{3}$ ANPD: E. A. Aitken, 1956 $4.45 \mathrm{gm} / \mathrm{cm}^{3}$ 


\begin{tabular}{|c|c|c|c|c|c|c|c|}
\hline TEMPERATURE: & $\cos F$ & $500 \mathrm{FF}$ & $1000 \% \mathrm{~F}$ & $1500 \% \mathrm{~F}$ & $2000 \mathrm{~F}$ & $25000 \mathrm{~F}$ & $3000 \mathrm{FF}$ \\
\hline$D_{1}$ & 1.081 & 1.095 & 1.104 & 1.111 & 1.116 & 1.121 & 1.124 \\
\hline$D_{2}$ & .1845 & .2366 & .2816 & .3171 & .3466 & .3717 & .3934 \\
\hline$\Sigma_{a_{1}}$ & .002614 & .002012 & .001679 & .001478 & .001339 & .001237 & .001156 \\
\hline$\Sigma_{a_{2}}$ & .04504 & .03339 & .02708 & .02337 & .02086 & .01902 & .01759 \\
\hline$\Sigma_{s_{1}}$ & .03010 & .03123 & .03195 & .03244 & .03281 & .03310 & .03335 \\
\hline$d$ & 1.538 & $1.44 \theta$ & 1.405 & 1.385 & 1.377 & 1.375 & 1.376 \\
\hline$d_{1}$ & 2.304 & 2.333 & 2.354 & 2.368 & 2.379 & 2.388 & 2.396 \\
\hline$d_{2}$ & .3932 & .5044 & .6002 & .6759 & .7389 & .7923 & .8385 \\
\hline$L L_{1}^{2}$ & 33.04 & 32.93 & 32.84 & 32.76 & 32.69 & 32.63 & 32.58 \\
\hline$L_{2}^{2}$ & 4.096 & 7.086 & 10.40 & 13.57 & 16.62 & 19.55 & 22.37 \\
\hline$p$ & .9201 & .9395 & .9501 & .9564 & .9608 & .9640 & .9665 \\
\hline
\end{tabular}

COMPOSITION BY WEIGHT FRACTION:

REFERENCE:

$$
\mathrm{H} .0195\left(\mathrm{~N}_{\mathrm{H}}=5 \times 10^{22}\right)
$$

ANPD: C. R. Simmons ANPD: C. R. Simmons

ASSUMEO DENSITY: $\quad 4.30 \mathrm{gm} / \mathrm{cm}^{\circ}$
MATERIAL: Hydrided Yttrium (H Gas) $\begin{array}{llll}15000^{\circ} & 20000^{\circ} & 2500^{\circ} \mathrm{F} & 3000^{\circ} \mathrm{F}\end{array}$

\begin{tabular}{|c|c|c|c|c|c|c|c|}
\hline TEMPERATURE: & GePF & $5000 \mathrm{~F}$ & $1000 \mathrm{~F}$ & $15000 \mathrm{~F}$ & $2000 \mathrm{~F}$ & $2500 \mathrm{~F}$ & $3000 \mathrm{~F}$ \\
\hline$D_{1}$ & 1. 096 & 1.106 & 1. 113 & 1. 118 & 1. 122 & 1.125 & 1,128 \\
\hline$D_{2}$ & .3218 & .3862 & .4285 & .4565 & .4771 & .4931 & .5063 \\
\hline$\Sigma_{a_{1}}$ & .002390 & .001907 & .001614 & .001426 & .001300 & .001205 & .001131 \\
\hline$\Sigma_{a_{2}}$ & .04504 & .03339 & .02708 & .02337 & .02086 & .01902 & .01759 \\
\hline$\Sigma_{s_{1}}$ & .03061 & .03153 & .03217 & .03263 & .03297 & .03324 & .03347 \\
\hline & & & & & & & \\
\hline$d$ & 1.668 & 1.612 & 1. 580 & 1.562 & 1.550 & 1.541 & 1.536 \\
\hline$d_{1}$ & 2. 336 & 2.357 & 2.372 & 2.383 & 2.392 & 2. 399 & 2. 405 \\
\hline$d_{2}$ & .6859 & .8231 & .9134 & .9731 & 1. 017 & 1.051 & 1.079 \\
\hline$L_{1}^{2}$ & 33.21 & 33.06 & 32.94 । & 32.83 & 32.75 & 32.67 & 32.61 \\
\hline$L_{2}^{2}$ & 7.145 & 11.56 & 15.83 & 19.53 & 22.87 & 25.93 & 28.79 \\
\hline$p$ & .9276 & .9430 & .9522 & .9581 & .9621 & .9650 & .9673 \\
\hline
\end{tabular}

COMPOSITION BY WEIGHT FRACTION:

DENSITY VARIATIONS AND REFERENCE:

ANPD: C. R. Simmons

$4.30 \mathrm{gm} / \mathrm{cm}^{3}$

REFERENCE

$\mathrm{Y} .9805$

$.0195\left(\mathrm{~N}_{\mathrm{H}}=5 \times 10^{22}\right)$

ANPD: C. R. Simmons 


\begin{tabular}{|c|c|c|c|c|c|c|c|}
\hline TEMPERATURE: & $60 \%$ & $500 \% \mathrm{~F}$ & $1000^{\circ} \mathrm{F}$ & $15000 \mathrm{~F}$ & $2000{ }^{\circ} \mathrm{F}$ & $25000 \mathrm{~F}$ & $3000 \% \mathrm{~F}$ \\
\hline$D_{2}$ & 1.012 & 1.025 & 1.035 & 1.041 & 1.046 & 1.050 & 1.054 \\
\hline$D_{2}$ & .1568 & .2021 & .2415 & .2729 & .2991 & .3215 & .3409 \\
\hline$\Sigma_{a_{1}}$ & .002783 & .002138 & .001784 & .001569 & .001421 & .001312 & .001227 \\
\hline$\Sigma_{a_{2}}$ & .04772 & .03538 & .02869 & .02476 & .02210 & .02015 & .01863 \\
\hline$\Sigma_{s_{1}}$ & .03636 & .03764 & .03847 & .03902 & .03945 & .03979 & .04007 \\
\hline$d$ & 1.368 & 1.281 & 1.237 & 1.217 & 1.209 & 1. 207 & 1.209 \\
\hline$d_{1}$ & 2.156 & 2.185 & 2.205 & 2.219 & 2.230 & 2.239 & 2.246 \\
\hline$d_{2}$ & .3343 & .4308 & .5148 & .5817 & .6375 & .6852 & .7267 \\
\hline$L \frac{2}{1}$ & 25.84 & 25.77 & 25.70 & 25.65 & 25.60 & 25.55 & 25.51 \\
\hline & & 5712 & 8418 & & & & \\
\hline$L \frac{2}{2}$ & 3.281 & 0.713 & 8.418 & 11.02 & 13.53 & 15.96 & 18.30 \\
\hline$p$ & .9289 & .9462 & .9557 & .9614 & .9652 & .9681 & .9703 \\
\hline
\end{tabular}

COMPOSITION GY WEIGHT FRACTION:

$\begin{array}{ll} & \mathrm{Y} .977 \\ \text { REFERENCE: } & \mathrm{H} .023 \quad\left(\mathrm{~N}_{\mathrm{H}}=6 \times 10^{22}\right) \\ & \text { ANPD: } \mathrm{C}, \mathrm{R}, \text { Simmons }\end{array}$
DENSITY VARIATIONS AND REFERENCE: ANPD: C. R. Simmons $4.28 \mathrm{gm} / \mathrm{cm}^{3}$

MATERIAL: Hydrided Yttrium (H Gas)

ASSUMED DENSITY; $\quad 4.28 \mathrm{sm} / \mathrm{FH}^{\circ}$

\begin{tabular}{|c|c|c|c|c|c|c|c|}
\hline TEMPERATÚRE: & $60^{\circ} \mathrm{F}$ & $500 \mathrm{~F}$ & $1000^{\circ} \mathrm{F}$ & $1500{ }^{\circ} \mathrm{F}$ & $2000 \mathrm{~F}^{-} \mathrm{l}$ & $2500 \mathrm{~F}$ & $3000^{\circ} \mathrm{F}$ \\
\hline$D_{1}$ & 1.026 & 1.035 & 1.042 & 1.048 & 1.051 & 1.055 & 1,058 \\
\hline$D_{2}$ & .2778 & .3352 & .3733 & .3986 & .4173 & .4318 & .4439 \\
\hline$\Sigma_{a,}$ & .002543 & .002027 & .001714 & .001513 & .001379 & .001278 & .001199 \\
\hline$\Sigma_{a_{2}}$ & .04772 & .03538 & .02863 & .02476 & .02210 & .02015 & .01863 \\
\hline$\Sigma_{s,}$ & .03695 & .03799 & .03872 & .03925 & .03963 & .03995 & .04021 \\
\hline$d$ & 1.491 & 1.434 & 1.403 & 1.385 & 1.373 & 1.366 & 1.360 \\
\hline$d_{1}$ & 2.187 & 2.207 & 2.222 & 2.233 & 2. 241 & 2.248 & 2.254 \\
\hline$d_{2}$ & .5921 & .7144 & .7956 & .8496 & .8894 & .9204 & .9461 \\
\hline$L_{1}^{2}$ & 25.98 & 25.87 & 25.78 & 25.70 & 25.64 & 25.59 & 25.54 \\
\hline$L_{2}^{2}$ & 5.821 & 9.474 & 13.01 & 16.10 & 18.88 & 21.43 & 23.82 \\
\hline$p$ & .9356 & .9494 & 9576 & .9629 & .9664 & .9690 & .9710 \\
\hline
\end{tabular}

COMPOSITION BY WEIGHT FRACTION:
DENSITY VARIATIONS AND REFERENCE: ANPD: C. R. Simmons $4.28 \mathrm{gm} / \mathrm{cm}^{3}$ 


\begin{tabular}{|c|c|c|c|c|c|c|c|}
\hline TEMPERATURE: & $60 \%$ & $500 \mathrm{~F}$ & $1000^{\circ} \mathrm{F}$ & $1500 \% \mathrm{~F}$ & $2000 \mathrm{~F}$ & $25000 \mathrm{~F}$ & $3000 \% \mathrm{~F}$ \\
\hline$D_{1}$ & .9379 & .9496 & .9576 & .9629 & .9669 & .9702 & .9728 \\
\hline$D_{2}^{\prime}$ & .2198 & .2765 & .3239 & .3603 & .3899 & .4146 & .4356 \\
\hline$\Sigma_{a_{1}}$ & .001072 & .0008187 & .0006810 & .0005978 & .0005410 & .0004990 & .0004663 \\
\hline$\Sigma_{a_{2}}$ & .01785 & .01323 & .01073 & .009260 & .008266 & .007536 & .006968 \\
\hline$\Sigma_{s_{1}}$ & .02521 & .02591 & .02638 & .02670 & .02695 & .02715 & .02732 \\
\hline$d$ & 1.103 & 1.074 & 1.081 & 1.099 & 1. 120 & 1. 141 & 1. 161 \\
\hline$d_{1}$ & 1.999 & 2.024 & 2.041 & 2.052 & 2.061 & 2.068 & 2.074 \\
\hline$d_{2}$ & .4685 & .5894 & .6904 & .7680 & .8311 & .8837 & .9285 \\
\hline$L_{1}^{2}$ & 35.69 & 35.53 & 35.39 & 35.28 & 35.18 & 35.09 & 35.01 \\
\hline$L \frac{2}{2}$ & 12.32 & 20.90 & 30.18 & 38.91 & 47.17 & 55.01 & 62.51 \\
\hline$p$ & .9592 & .9694 & .9748 & .9781 & .9803 & .9820 & .9832 \\
\hline
\end{tabular}

COMPOSITION BY WEIGHT FRACTION:

$$
\begin{array}{ll}
\mathrm{Zr} & .9886 \\
\mathrm{H} & .0114\left(\mathrm{~N}_{\mathrm{H}}=4 \times 10^{22}\right)
\end{array}
$$

REFERENCE:
DENSITY VARIATIONS AND REFERENCE: ANPD: C. R. Simmons, $19575.89 \mathrm{gm} / \mathrm{cm}^{3}$ ANPD: E. A. Aitken, $1956 \quad 6.05 \mathrm{gm} / \mathrm{cm}^{3}$

ANPD: C. R. Simmons

material: Hydrided Zirconium (H Gas)

\begin{tabular}{|c|c|c|c|c|c|c|c|}
\hline TEMPERATURE: & $\infty 0 \% F$ & $5000 \mathrm{~F}$ & $1000^{\circ} \mathrm{F}$ & $15000 \mathrm{~F}$ & $20000 \mathrm{~F}$ & $2500^{\circ} \mathrm{F}$ & $3000{ }^{\circ} \mathrm{F}$ \\
\hline$D_{1}$ & .9522 & .9597 & .9651 & 9690 & .9719 & .9743 & .9763 \\
\hline$D_{2}$ & .3704 & 4335 &, 4732 & .4988 & .5172 & .5313 &, 5429 \\
\hline$\Sigma_{a_{1}}$ & .0009765 & .0007750 &, 0006539 & .0005765 & .0005248 & .0004861 & .0004556 \\
\hline$\Sigma_{a_{2}}$ & .01785 & .01323 & .01073 & .009260 & .008266 & .007536 & .006968 \\
\hline$\Sigma_{s_{1}}$ &, 02555 &, 0261.2 & .02653 & .02683 & .02706 & .02725 & .02740 \\
\hline$d$ & 1.300 & 1,301 & 1.311 & 1.320 & 1.329 & 1.337 & 1.344 \\
\hline$d_{1}$ & 2,030 & 2,046 & 2,057 & 2.065 & 2.072 & 2.077 & 2.081 \\
\hline$d_{2}$ & .7896 &, 9239 & 1,009 & 1,063 & 1. 102 & 1. 132 & 1. 157 \\
\hline$L_{1}^{2}$ & 35,90 & 35.68 & 35,50 & 35.35 & 35.23 & 35.13 & 35.04 \\
\hline$L_{2}^{2}$ & 20.76 & 32,76 & 44.10 & 53.86 & 62.57 & 70.50 & 77.91 \\
\hline$p$ & 9632 & .9712 & 9759 & .9790 & .9810 & 9825 & .9836 \\
\hline
\end{tabular}

ASSUMED DENSITY: $\quad 5.89 \mathrm{gm} / \mathrm{cm}^{\circ}$
COMPOSITION BY WEIGHT FRACTION:

$$
\begin{array}{ll}
\mathrm{Zr} & .9886 \\
\mathrm{H} & .0114\left(\mathrm{~N}_{\mathrm{H}}=4 \times 10^{22}\right)
\end{array}
$$

'REFERENCE:
DENSITY VARIATIONS AND REFERENCE: ANPD: C. R. Simmons, $19575.89 \mathrm{gm} / \mathrm{cm}^{3}$ ANPD: E. A. Aitken, 1956 


\begin{tabular}{|c|c|c|c|c|c|c|c|}
\hline TEMPERATURE: & $60 \%$ & $5000 \mathrm{~F}$ & $1000 \mathrm{~F}$ & $1500 \mathrm{~F}$ & $2000{ }^{\circ} F$ & $2500 \mathrm{~F}$ & $3000 \% \mathrm{~F}$ \\
\hline$D_{1}$ & .8969 & .9086 & .9167 & .9221 & .9262 & .9296 & .9323 \\
\hline$D_{2}$ & .1822 & .2315 & .2733 & .3060 & .3328 & .3554 & .3747 \\
\hline$\Sigma_{a_{1}}$ & .001235 & .0009424 & .0007837 & .0006879 & .0006224 & .0005741 & .0005363 \\
\hline$\Sigma_{a_{2}}$ & .02058 & .01526 & .01237 & .01068 & .009531 & .008689 & .008034 \\
\hline$\Sigma_{s_{1}}$ & .03146 & .03231 & .03288 & .03327 & .03357 & .03382 & .03403 \\
\hline$d$ & .9908 & .9564 & .9576 & .9712 & .9890 & 1.008 & 1.026 \\
\hline$d_{1}$ & 1.912 & 1.937 & 1.954 & 1.965 & 1.974 & 1. 981 & 1.987 \\
\hline$d_{2}$ & .3884 & .4935 & .5826 & .6522 & .7093 & .7574 & .7987 \\
\hline$L_{1}^{2}$ & 27.43 & 27.32 & 27.23 & 27.15 & 27.09 & 27.03 & 26.97 \\
\hline$L_{2}^{2}$ & 8.856 & 15.17 & 22.09 & 28.66 & 34.92 & 40.90 & 46.64 \\
\hline$p$ & .9622 & .9717 & .9767 & .9797 & .9818 & .9833 & .9845 \\
\hline
\end{tabular}

COMPOSITION GY WEIGHT FRACTION:

$\begin{array}{ll} & \mathrm{Zr} .9854 \\ \text { REFERENCE: } & \mathrm{H} .0146\left(\mathrm{~N}_{\mathrm{H}}=5 \times 10^{22}\right) \\ & \text { ANPD: } \mathrm{C} . \mathrm{R} . \text { Simmons }\end{array}$
DENSITY VARIATIONS AND REFERENCE: ANPD: C. R. Simmons $5.72 \mathrm{gm} / \mathrm{cm}^{3}$

$$
\text { material: HydTided Zitconjum }
$$

ASSUMEO DENSITY: $\quad 5.72 \mathrm{gm}^{\mathrm{m}} \mathrm{cm}$

\begin{tabular}{|c|c|c|c|c|c|c|c|}
\hline TEMPERATURE: & $60 \%$ & $500 \% \mathrm{~F}$ & $1000 \mathrm{~F}$ & $1500 \% \mathrm{~F}$ & $2000 \mathrm{~F}$ & $2500 \mathrm{~F}$ & $3000 \mathrm{~F}$ \\
\hline$D_{1}$ & .9106 &, 9183 & .9240 & .9280 & .9311 & .9336 & .9358 、 \\
\hline$D_{2}$ & .3150 & .3727 & $=4097$ & .4338 & .4513 & .4647 & .4758 \\
\hline$\Sigma_{a_{1}}$ & .001124 & .0008920 & .0007525 & .0006632 & $=0006037$ & .0005592 & 0005241 \\
\hline$\Sigma_{a_{2}}$ & .02058 & .01526 & .01237 & .01068 & 009531 & .0008689 &, 008034 \\
\hline$\Sigma_{s_{1}}$ & 03188 & .03257 & 03307 & .03344 & 03371 & 03394 & .03414 \\
\hline$d$ & 1,169 & 1. 165 & 1.172 & 1. 180 & 1,187 & $1: 194$ & 1, 201 \\
\hline$-d_{1}$ & 1,941 & 1.957 & 1.970 & 1,978 & 1985 & 1. 990 & 1.995 \\
\hline$d_{2}$ & .6714 & 7944 & 8734 & .9247 & .9619 & 9905 & 1,014 \\
\hline$L_{1}^{2}$ & $27: 59$ & 27.45 & 27,32 & 27.21 & 27,13 & 27.06 & 27.00 \\
\hline$L_{2}^{2}$ & 15,31 & $24: 43$ & $33: 11$ & 40.63 & 47.35 & 53,48 & 59.22 \\
\hline$p$ & 9659 & : 9733 & 9778 & .9806 &, 9824 & $=9838$ & 9849 \\
\hline
\end{tabular}

COMPOSITION BY WEIGHT FRACTION:

$\mathrm{Zr}, 9854$

REFERENCE:

$$
\mathrm{H} \quad .0146\left(\mathrm{~N}_{\mathrm{H}}=5 \times 10^{22}\right)
$$

ANPD: C. R. Simmons
DENSITY VARIATIONS AND REFERENCE: ANPD: C. R. Simmons $5,72 \mathrm{gm} / \mathrm{cm}^{3}$ 
MATERIAL: INCONEL ' $\mathrm{X}$ "

Note: Mocked up Co with Mn.

ASSUMED DENSITY: $8.310 \mathrm{gm}^{\mathrm{cm}} \mathrm{cm}^{\mathrm{s}}$,

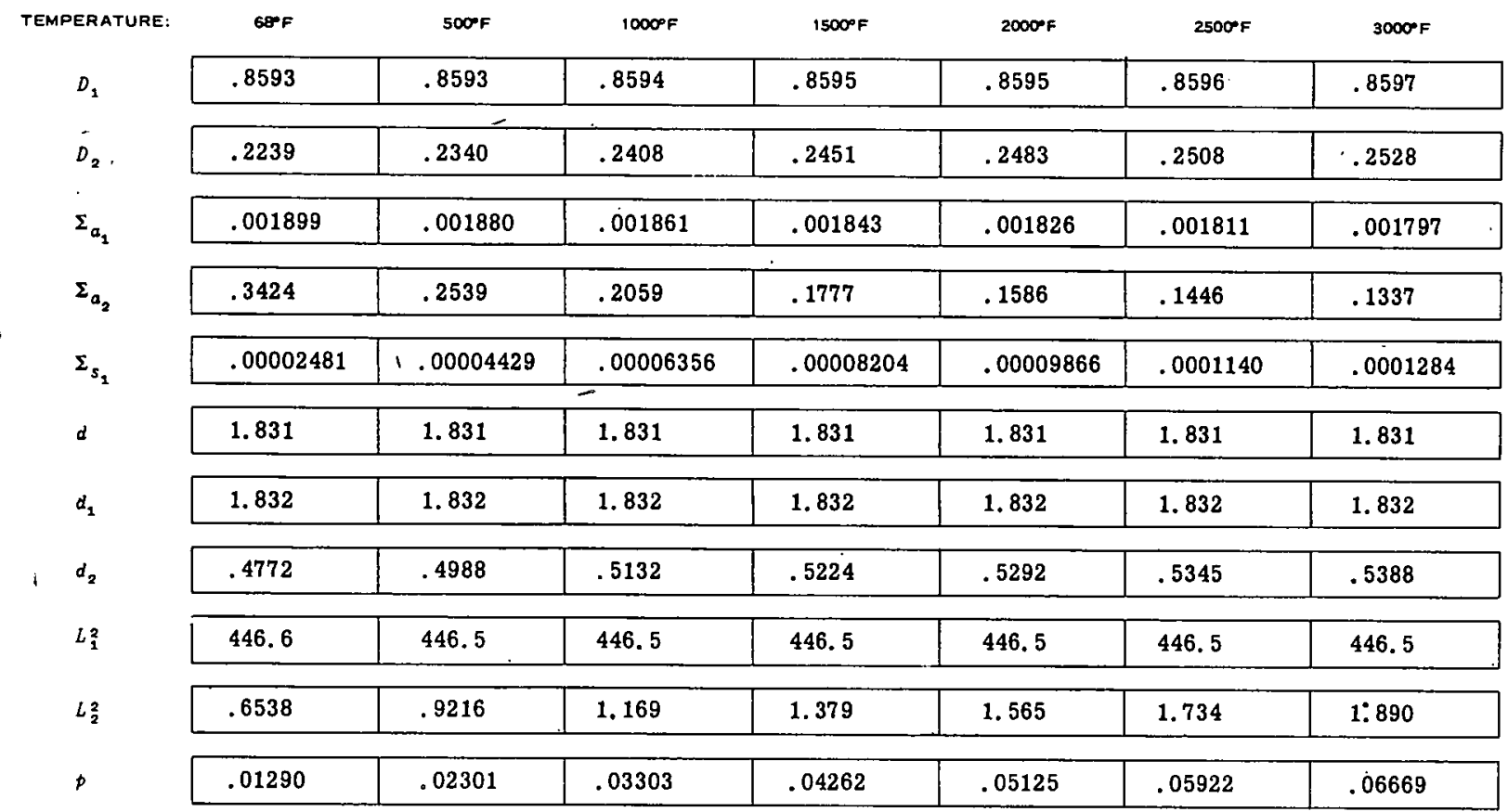

COMPOSITION BY WEIGHT FRACTION:

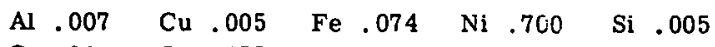

REFERENCE:

Co.01 $\mathrm{Cr} .155 \mathrm{Mn} .01 \quad \mathrm{Nb} .009 \quad \mathrm{Ti} .025$

ANPD: F. D. Kingsbury
DENSITY VARIATIONS AND REFERENCE:

ANPD: F, D. Kingsbury

$8.31 \mathrm{gm} / \mathrm{cm}^{3}$

MATERIAL: Indium

TEMPERATURE:

$68^{\circ} \mathrm{F}$

$500^{\circ} \mathrm{F}$

$1000 \mathrm{~F}^{\circ}$

$1500^{\circ} \mathrm{F}$

ASSUMED DENSITY:

$7.310 \mathrm{gm}^{\mathrm{cm}} \mathrm{cm}^{9}$

\begin{tabular}{|c|c|c|c|c|c|c|c|}
\hline ERATURE: & $68^{\circ} \mathrm{F}$ & $500^{\circ} \mathrm{F}$ & $1000^{\circ} \mathrm{F}$ & $1500^{\circ} \mathrm{F}$ & $2000^{\circ} \mathrm{F}$ & $2500{ }^{\circ} \mathrm{F}$ & $3000^{\circ} \mathrm{F}$ \\
\hline \multirow[t]{2}{*}{$D_{1}$} & 1.705 & 1.705 & 1.705 & 1.705 & 1.705 & 1. 705 & 1.705 \\
\hline & 1 & & & & & & \\
\hline \multirow[t]{2}{*}{$D_{2}$} & .04944 & .06320 & .07266 & .07789 & .07950 & .07687 & .06962 \\
\hline & & & & & & 2 & \\
\hline \multirow{2}{*}{$\Sigma_{a_{1}}$} & .004720 & .004720 & .004720 & .004720 & .004720 & .004720 & .004720 \\
\hline & & & & & 1 & & \\
\hline$\Sigma_{a}$ & 6.598 & 5. 125 & 4.436 & -4.126 & 4. 033 & 4. 164 & 4. 592 \\
\hline
\end{tabular}

\begin{tabular}{l|l|l|l|l|l|l|l|}
\hline$s_{s_{1}}$ & $9.247 \times 10^{-16}$ & $1.876 \times 10^{-15}$ & $2.876 \times 10^{-15}$ & $4.104 \times 10^{-15}$ & $5.282 \times 10^{-15}$ & $6.412 \times 10^{-15}$ & $7.514 \times 10^{-15}$ \\
\hline
\end{tabular}

\begin{tabular}{|l|l|l|l|l|l|l|}
\hline 3.634 & 3.634 & 3.634 & 3.634 & 3.634 & 3.634 & 3.634 \\
\hline
\end{tabular}

\begin{tabular}{|l|l|l|l|l|l|l|l|}
\hline$d_{1}$ & 3.634 & 3.634 & 3.634 & 3.634 & 3.634 & 3.634 \\
\hline
\end{tabular}

\begin{tabular}{|c|c|c|c|c|c|c|}
\hline .1054 & .1347 & .1549 & .1660 & .1695 & .1638 & .1484 \\
\hline
\end{tabular}

\begin{tabular}{|l|l|l|l|l|l|l|l|}
\hline$L_{1}^{2}$ & 361.1 & 361.1 & -361.1 & 361.1 & 361.1 & 361.1 \\
\hline
\end{tabular}

\begin{tabular}{l|l|l|l|l|l|l|l|}
\hline$L \frac{2}{2}$ & .007493 & .01233 & .01638 & .01888 & .01971 & .01846 \\
\hline
\end{tabular}

\begin{tabular}{ll|l|l|l|l|l|l|l|}
\hline $1.959 \times 10^{-13}$ & $3.975 \times 10^{-13}$ & $6.093 \times 10^{-13}$ & $6.694 \times 10^{-13}$ & $1.119 \times 10^{-12}$ & $1.358 \times 10^{-12}$ & $1.592 \times 10^{-12}$ \\
\hline
\end{tabular}

COMPOSITION BY WEIGHT FRACTION:

In 1.00
DENSITY VARIATIONS AND REFERENCE:

$\mathrm{RMH}$

$7.31 \mathrm{gm} / \mathrm{cm}^{3}$

REFERENCE 


\begin{tabular}{|c|c|c|c|c|c|c|c|}
\hline TEMPERATURE: & $\cos F$ & SocrF & $1000 \mathrm{~F}$ & $1500 \mathrm{~F}$ & $2000 \% \mathrm{~F}$ & $2500 \mathrm{FF}$ & $3000 \mathrm{~F}$ \\
\hline$n_{1}$, & .9977 & .9978 & .9979 & .9980 & .9982 & .9983 & .9984 \\
\hline$D_{2}$ & .3087 & .3116 & .3233 & .3289 & .3326 & .3349 & .3366 \\
\hline$\Sigma_{a_{i}}$ & .001275 & .001256 & .001238 & .001222 & .001207 & .001193 & .001181 \\
\hline$\Sigma_{a_{2}}$ & .1905 & .1412 & .1145 & .09885 & .08823 & .08043 & .07439 \\
\hline$\Sigma_{s_{1}}$ & .00002694 & .00004603 & .00006440 & .00008129 & .00009632 & .0001100 & .0001227 \\
\hline$d$ & 2.126 & 2.126 & 2.126 & 2. 126 & 2.126 & 2.126 & 2.126 \\
\hline$d_{1}$ & 2. 127 & 2.127 & 2.127 & 2.127 & 2.128 & 2.128 & 2.128 \\
\hline$d_{2}$ & .6580 & .6642 & .6892 & .7010 & .7090 & .7139 & .7174 \\
\hline$L \stackrel{2}{1}$ & 766.2 & 766.2 & 766.1 & 766.1 & 766.0 & 766.0 & 765.9 \\
\hline$L \frac{2}{2}$ & 1.620 & 2.206 & 2.823 & 3.327 & 3.770 & 4.164 & 4.524 \\
\hline$p$ & .02069 & .03534 & .04944 & .06240 & .07392 & .08442 & .09414 \\
\hline
\end{tabular}

COMPOSITION BY WEIGHT FRACTION:

Fe 1.00

REFERENCE:
DENSITY VARIATIONS AND REFERENCE:

MH $\quad 7.87 \mathrm{gm} / \mathrm{cm}^{3}$

$\mathrm{HCP} \quad 7.85-7.88 \cdot \mathrm{gm} / \mathrm{cm}^{3}$

$\mathrm{RH} \quad 7.87 \mathrm{gm} / \mathrm{cm}^{3}$
MAterial: Iron-Chrome-Aluminum
ASSUMED DENSITY: $\quad 7.200 \mathrm{~cm} / \mathrm{cm}^{3}$

\begin{tabular}{|c|c|c|c|c|c|c|c|}
\hline TEMPERATURE: & $68 \% F$ & $500 \% \mathrm{~F}$ & $1000^{\circ} \mathrm{F}$ & $1500^{\circ} \mathrm{F}$ & $2000 \% \mathrm{~F}$ & $25000 \mathrm{~F}$ & $3000 \mathrm{~F}$ \\
\hline$D_{1}$ & 1.127 & 1.127 & 1.127 & 1.128 & 1.128 & 1.128 & 1.128 \\
\hline$D_{2}$ & .3939 & .4038 & .4206 & .4293 & .4353 & .4393 & .4423 \\
\hline$\Sigma_{a_{1}}$ & .001252 & .001238 & .001225 & .001212 & .001200 & .001189 & .001179 \\
\hline$\Sigma_{a_{2}}$ & .1773 & .1314 & .1066 & .09198 & .08210 & .07484 & .06923 \\
\hline$\Sigma_{s_{1}}$ & .00001902 & .00003322 & .00004708 & .00006021 & .00007202 & .00008288 & .00009300 \\
\hline$d$ & 2.403 & 2.403 & 2.403 & 2.402 & 2.402 & 2.402 & 2.402 \\
\hline$d_{1}$ & 2.403 & 2.403 & 2.403 & 2.403 & 2.404 & 2.404 & 2.404 \\
\hline$d_{2}$ & .8396 & .8608 & .8966 & .9150 & .9279 & .9364 & .9427 \\
\hline$L_{1}^{2}$ & 886.7 & 886.6 & 886.6 & 886.5 & 886.5 & 886.4 & 886.4 \\
\hline$L_{2}^{2}$ & 2.222 & 3.072 & 3.947 & 4. 667 & 5.302 & 5.870 & $6.38 y$ \\
\hline$p$ & .01496 & .02612 & .03702 & .04734 & .05661 & .06514 & .07309 \\
\hline
\end{tabular}

COMPOSITION OY WEIGHT FRACTION:

$\begin{array}{lll} & \mathrm{Fe} & .70 \\ & \mathrm{Cr} & .25 \\ \text { REFERENCE: } & \mathrm{Al} & .05\end{array}$

ANPD: E. A. Aitken
DENSITY VARIATIONS AND REFERENCS:

ANPD: É. A. Aitken

$7.2 \mathrm{gm} / \mathrm{cm}^{3}$ 


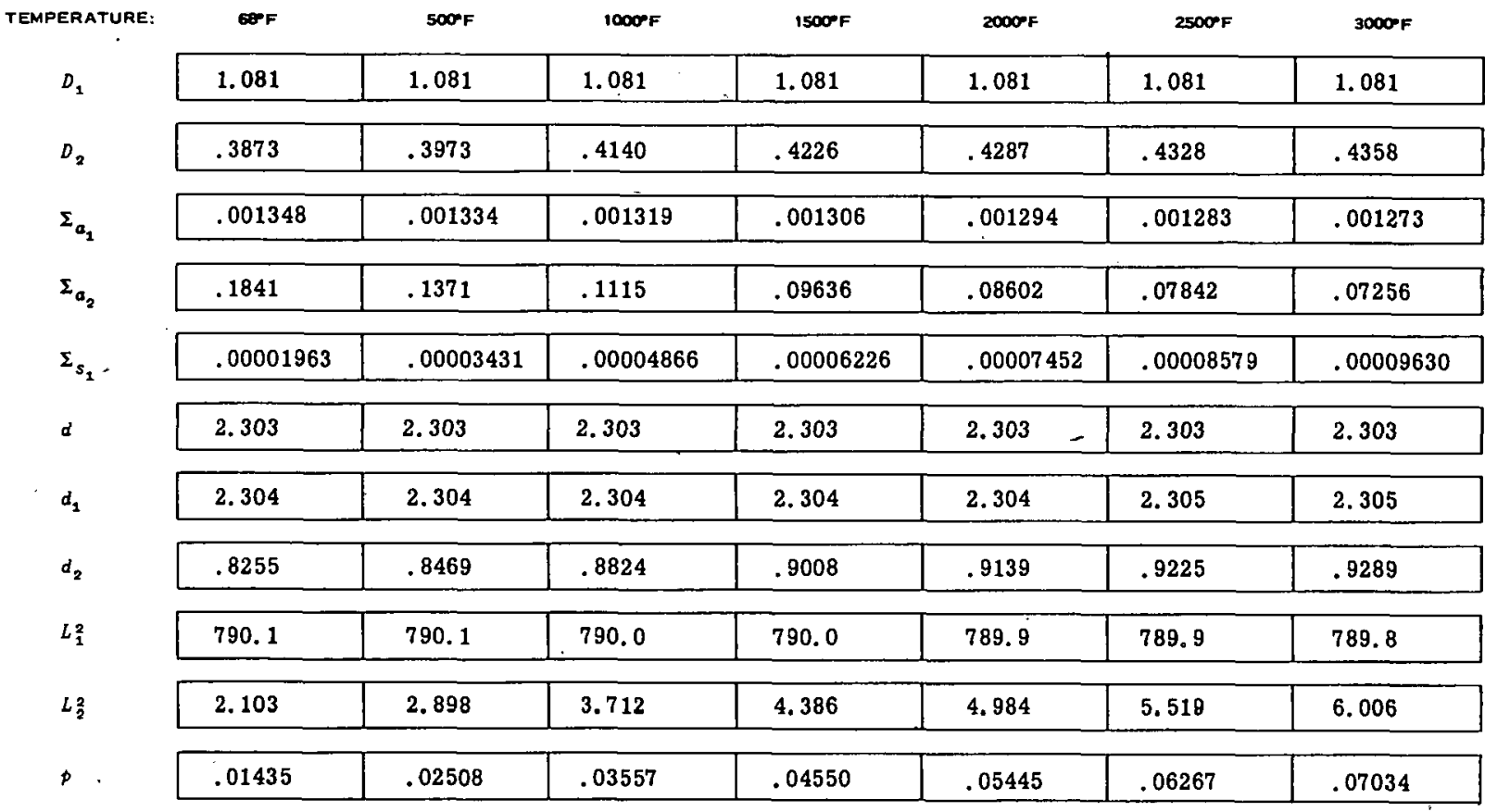

COMPOSITION OY WEIGHT FRACTION:

REFERENCE:
DENSITY VARIATIONS AND REFERENCE:
ANPD

$7.2 \mathrm{gm} / \mathrm{cm}^{3}$

ANPD

Material: Nickel-chromium alloy (Nb Modified)
ASSUMEO DENSITY

$8.440 \mathrm{gm} / \mathrm{cm}^{\mathrm{s}}$

TEMPERATURE

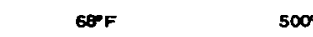

$10000^{\circ} \mathrm{F}$

$1500{ }^{\circ} \mathrm{F}$

$2000^{\circ} \mathrm{F}$

$2500^{\circ} \mathrm{F}$

$3000 \mathrm{~F}$

$D_{1}$

.8624

.8625

.8626

.8626

.2353

.2381

\begin{tabular}{|c|c|}
\hline .8627 & .8628 \\
\hline .2403 & .2421 \\
\hline
\end{tabular}

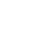

.216

$\Sigma_{a_{1}}$.

\begin{tabular}{|l|l|l|l|l|l|l|}
\hline .001841 & .001820 & .001799 & .001780 & .001762 & .001746 & .001731 \\
\hline
\end{tabular}

$\Sigma_{a_{2}}$

.3296

.2444

.1982

\begin{tabular}{|l|l|l|l|l|l|l|}
\hline .00002767 & .00004896 & .00006993 & .00008974 & .0001075 & .0001238 & .0001390 \\
\hline
\end{tabular}

$\Sigma_{s_{1}}$

$$
\text { (1.83) }
$$

$d$

1.838

1.838

1.838

1.838

1.838

1.838

1.838

1.838

1. 839

1. 839

1. 839

1.838

$d_{1}$

\begin{tabular}{|l|l|}
\hline 1.838 & 1.83 \\
\hline
\end{tabular}

1.838

.5016

.4937 -

.5016

.5075

.5121

461.3

461.3

461.3

$L_{1}^{2}$

\begin{tabular}{|l|l|l|}
\hline 461.4 & 461.4 & 461 \\
\hline
\end{tabular}

$L \frac{2}{2}$

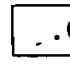

p

\begin{tabular}{|l|l|l}
\hline .01480 & .02619 & .03 \\
\hline
\end{tabular}

1.169

1.376

1.559

1.726

1.880

COMPOSITION BY WEIGHT FRACTION:

$$
\begin{array}{lllllll}
\mathrm{Cr} & .195 & \mathrm{Si} .009 & \mathrm{Mn} & .005 & \mathrm{Ni} & .77 \\
\mathrm{Nb} & .01 & \mathrm{Fe} .01 & \mathrm{Cu} & .001 & &
\end{array}
$$

REFERENCE:

ANPD: F. D. Kingsbury 


\begin{tabular}{|c|c|c|c|c|c|c|c|}
\hline TEMPERATURE: & corf & soor & $1000 \mathrm{~F}$ & $1500 \% \mathrm{~F}$ & $2000 \mathrm{~F}$ & $2500 \mathrm{~F}$ & $3000 \mathrm{~F}$ \\
\hline$D_{1}$ & 1.146 & 1.147 & 1.147 & $1: 148$ & 1.149 & 1.149 & 1.150 \\
\hline$D_{2}$ & 1.165 & 1.036 & .9874 & .9647 & .9516 & .9430 & .9370 \\
\hline$\Sigma_{a_{1}}$ & .0001708 & .0001565 & .0001449 & .0001370 & .0001305 & .0001251 & .0001205 \\
\hline$\Sigma_{a_{2}}$ & .004976 & .003689 & .002993 & .002581 & .002303 & .002102 & .001944 \\
\hline$\Sigma_{s_{1}}$ & .00003625 & .00005135 & .00006367 & .00007230 & .00007944 & .00008543 & .00009058 \\
\hline$d$ & 2.444 & 2.441 & 2.438 & 2.437 & 2.435 & 2.433 & 2.431 \\
\hline$d_{1}$ & 2.443 & 2.444 & 2.445 & 2.447 & 2.449 & 2.450 & 2.451 \\
\hline$d_{2}$ & 2.484 & 2.209 & 2.105 & 2.056 & 2.028 & 2.010 & 1.997 \\
\hline$L_{1}^{2}$ & 5536. & 5517. & 5499. & 5485. & 5472. & 5460. & 5449. \\
\hline$L_{2}^{2}$ & 234.2 & 280.9 & 329.9 & 373.8 & 413.1 & 448.6 & 482.1 \\
\hline$p$ & .1751 & .2471 & .3052 & .3454 & .3784 & .4058 & .4292 \\
\hline
\end{tabular}

COMPOSITION BY WEIGHT FRACTION:

Pb 1.00

REFERENCE:
DENSITY VARIATIONS AMD REFEREMCE

HCP $11.35 \mathrm{gm} / \mathrm{cm}^{3}$

RH $\quad 11.34 \mathrm{gm} / \mathrm{cm}^{3}$
Material: Lithium
ASSUMEO DENSITY: $\quad .530 \mathrm{gm} / \mathrm{cm}^{\circ}$

$\begin{array}{llll}1500 \mathrm{~F} & 2000 \mathrm{~F} & 3000 \mathrm{~F}\end{array}$

TEMPERATURE: =

$D_{1}$

500F

$1000 \mathrm{~F}$

5.285

5.285

5.285

5.285

5.285

5.285

$D_{2}$

\begin{tabular}{l|l}
\hline .1133 & .1519 \\
\hline
\end{tabular}

\begin{tabular}{l|l}
1519 & .1865 \\
\hline
\end{tabular}

.2152

2402

.2627

003292

.003292

.003292

.003292

.003292

1. 342 .

1, 223

$\Sigma_{a_{2}}$

2.897

2.148

1. 742

J. 503

\begin{tabular}{|l|l|l|l|l|l|l|l|}
$1.962 \times 10^{-8}$ & $3.956 \times 10^{-8}$ & $6.047 \times 10^{-8}$ & $8.581 \times 10^{-8}$ & $1.101 \times 10^{-7}$ & $1.333 \times 10^{-7}$ & $1.559 \times 10^{-7}$ \\
\hline
\end{tabular}

$\mathbf{s}_{s_{1}}$

d

11.2

\begin{tabular}{l|l}
11.27 & 11.27
\end{tabular}

11.27

11. 27

11.27

1.1. 27

$d_{1}$

11.2

\begin{tabular}{l|l}
11.27 & 11.27
\end{tabular}

11.27

11.27

11.27

11.27

$d_{2}$

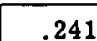

.3238

.3975

.4586

.5120

.5599

1605.

1605.

1605.

$$
L \frac{2}{2}
$$

\begin{tabular}{|l|l|l|}
\hline .03910 & .07072 & .1070 \\
\hline
\end{tabular}

$$
p
$$

.00001201

.00001837

.1431

.1790

\begin{tabular}{l|l}
.00002606 & .00003343 \\
\hline
\end{tabular}

COMPOSITION BY WEIGHT FRACTION:

Li 1.00

REFERENCE:

r 


\begin{tabular}{|c|c|c|c|c|c|c|c|}
\hline TEMPERATURE: & CerF & $500 \% F$ & $1000 \mathrm{~F}$ & $15000 \mathrm{~F}$ & $2000 \mathrm{~F}$ & $25000 \mathrm{~F}$ & $3000 \mathrm{~F}$ \\
\hline$D_{1}$ & 5.063 & 5.063 & 5.063 & 5.063 & 5.063 & 5.063 & 5.063 \\
\hline$D_{2}$ & .008589 & .01159 & .01435 & .01669 & .01877 & .02066 & .02242 \\
\hline$\Sigma_{a_{1}}$ & .01755 & .01755 & .01755 & .01755 & .01755 & .01755 & .01755 \\
\hline$\Sigma_{a_{2}}$ & 38.56 & 28.50 & 22.99 & 19.73 & 17.52 & 15.89 & 14.62 \\
\hline$\Sigma_{s_{1}}$ & $1.129 \times 10^{-13}$ & $2.285 \times 10^{-13}$ & $3.498 \times 10^{-13}$ & $4.980 \times 10^{-13}$ & $6.400 \times 10^{-13}$ & $7.760 \times 10^{-13}$ & $9.087 \times 10^{-13}$ \\
\hline$d$ & 10.79 & 10.79 & 10.79 & 10.79 & 10.79 & 10.79 & 10.79 \\
\hline$d_{1}$ & 10.79 & 10.79 & 10.79 & 10.79 & 10.79 & 10.79 & 10.79 \\
\hline$d_{2}$ & .01831 & .02471 & .03058 & .03557 & .04000 & .04404 & .04779 \\
\hline$L_{1}^{2}$ & 288.5 & 288.5 & 288.5 & 288.5 & 288.5 & 288.5 & 288.5 \\
\hline$L \frac{2}{2}$ & .0002227 & .0004068 & .0006241 & .0008458 & .001071 & .001301 & .001533 \\
\hline$p$ & $6.433 \times 10^{-12}$ & $1.302 \times 10^{-11}$ & $1.993 \times 10^{-11}$ & $2.837 \times 10^{-11}$ & $3.646 \times 10^{-11}$ & $4.422 \times 10^{-11}$ & $5.177 \times 10^{-11}$ \\
\hline
\end{tabular}

COMPOSITION BY WEIGHT FRACTION:

$$
\text { Li6 } 1.00
$$

REFERENCE:
DENSITY VARIATIONS AND REFERENCE:

MH $.46 \mathrm{gm} / \mathrm{cm}^{3}$ (computed)
MATERIAL: Lithium Ḣydride ( $\mathrm{H}$ Bound)
ASSUMED OENSITY: $\quad .749 \mathrm{gm}^{\mathrm{m}} / \mathrm{cm}^{\mathrm{s}}$

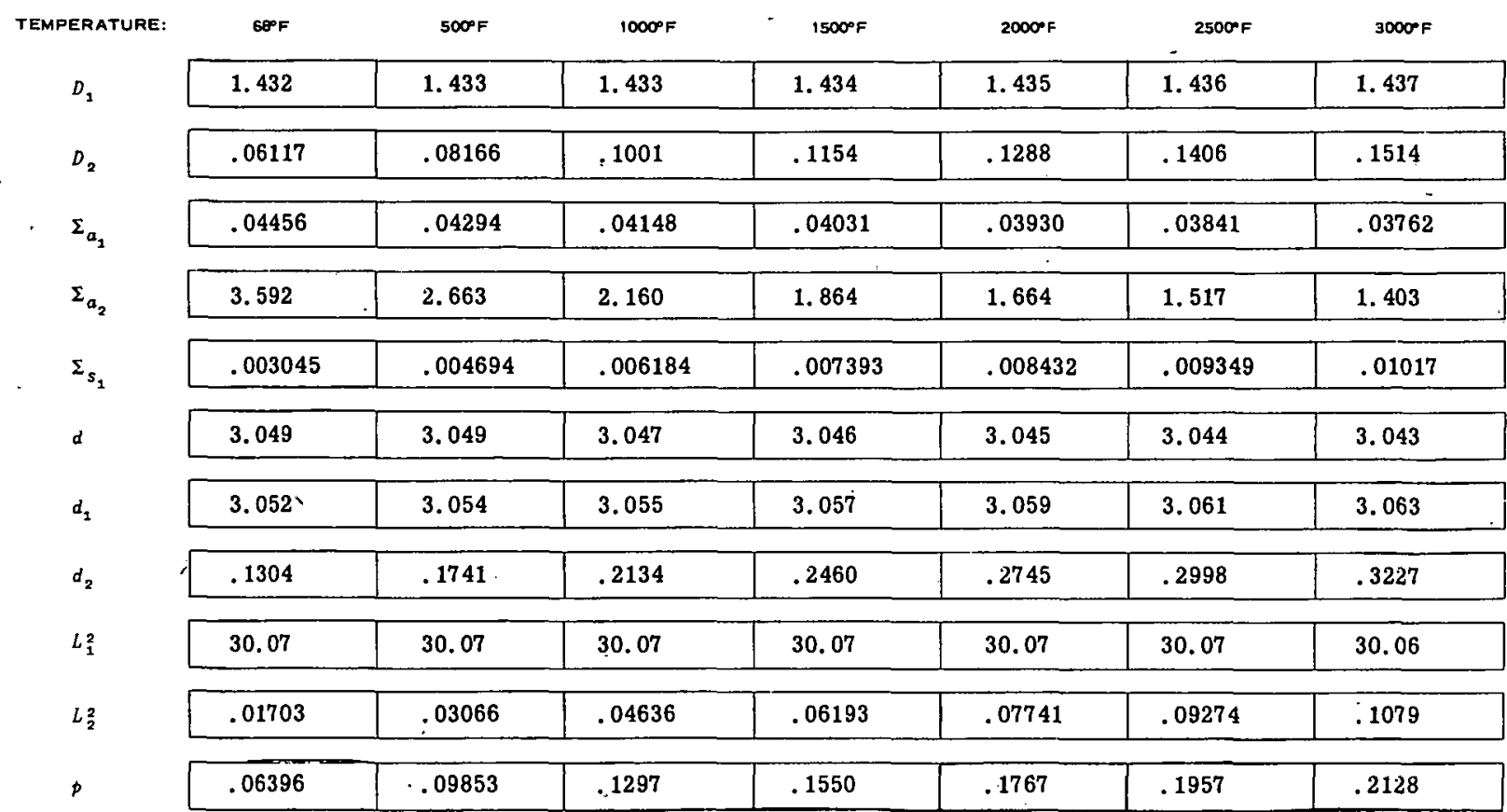

COMPOSITION BY WEIGHT FRACTION:
Li . 8732

H .1268
DENSITY VARIATIONS AND REFERENCE: ANPD: W. E. Edwards, \& M. A. Capo $\quad .749 \mathrm{gm} / \mathrm{cm}^{3}$ ANPD: E, A. Aitken $.780 \mathrm{gm} / \mathrm{cm}^{3}$ 


\begin{tabular}{|c|c|c|c|c|c|c|c|}
\hline TEMPERATURE: & $60 \%$ & $500 \% \mathrm{~F}$ & $1000 \mathrm{~F}$ & $1500^{\circ} \mathrm{F}$ & $2000^{\circ} \mathrm{F}$ & $25000 \mathrm{~F}$ & $3000 \mathrm{~F}$ \\
\hline$D_{1}$ & 1.432 & 1.433 & 1.434 & 1.435 & 1.436 & 1. 437 & 1. 437 \\
\hline$D_{2}$ & .07288 & .09628 & $: 1162$ & .1321 & .1456 & .1573 & .1677 \\
\hline$\Sigma_{a_{1}}$ & .04436 & .04274 & .04129 & .04012 & .03913 & .03825 & .03747 \\
\hline$\Sigma_{a_{2}}$ & 3.592 & 2.663 & 2.160 & 1.864 & 1.664 & 1.517 & 1.403 \\
\hline$\Sigma_{s_{1}}$ & .003275 & .004922 & .006404 & .007613 & .008636 & .009537 & .01034 \\
\hline$d$ & 3.051 & 3.050 & 3.049 & 3.048 & 3.046 & 3.045 & 3.044 \\
\hline$d_{1}$ & 3.053 & 3.055 & 3.057 & 3.059 & 3.061 & 3.062 & 3.064 \\
\hline$d_{2}$ & .1554 & .2052 & .2477 & .2816 & .3103 & .3352 & .3576 \\
\hline$L_{1}^{2}$ & 30.07 & 30.07 & 30.07 & 30.07 & 30.06 & 30.06 & 30.06 \\
\hline$L \frac{2}{2}$ & .02029 & .03615 & .05380 & .07088 & .08750 & .1037 & .1196 \\
\hline$p$ & .06875 & .1033 & .1343 & .1595 & .1808 & .1996 & .2163 \\
\hline
\end{tabular}

COMPOSITION GY WEIGHT FRACTION:

$$
\text { Li . } 8732
$$$$
\text { H . } 1268
$$

DENSITY VARIATIONS ANC REFERENCE: ANPD: W. E. Edwards \& M. A. Capo

$749 \mathrm{gm} / \mathrm{cm}^{3}$ ANPD: E. A. Aitken $.780 \mathrm{gm} / \mathrm{cm}^{3}$ 3

MATERIAL: Lithium OXide ASSUMED DENSITY: $\quad 1.812 \mathrm{gm}^{-\mathrm{cm}^{3}}$

TEMPERATÚRE:

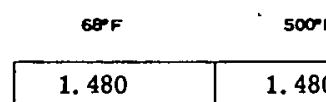

$D_{1}$

sor F

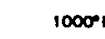

$\quad 1500 \%$

480

$2000 \%$

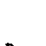

.06916

1. 480

1.480

1.480

-

2500\%

$3000^{\circ} \mathrm{F}$

$D_{2}$

.006989

.09200

.1121

.1285

.1427

1. 480

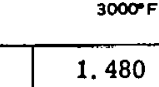

$\Sigma_{a_{1}}$

4.601

.006989

.006988

.006988

.006988

$\mid .1552$

.1666

$\Sigma_{a}$

$\Sigma_{s_{1}}$

\begin{tabular}{|l|l|l|l|l|l|l|}
\hline $2.065 \times 10^{-7}$ & $4.143 \times 10^{-7}$ & $6.318 \times 10^{-7}$ & $8.925 \times 10^{-7}$ & $1.142 \times 10^{-6}$ & $1.380 \times 10^{-6}$ & $1.612 \times 10^{-6}$ \\
\hline
\end{tabular}

$d$

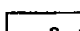

\begin{tabular}{|l|l|l|}
3.154 & 3.154 & 3.1 \\
\hline
\end{tabular}

3.154

3.154

3.154

3.154

3.154

$d_{1}$

\begin{tabular}{|l|l|}
\hline 3.154 & 3.15 \\
\hline
\end{tabular}

3.154

3.154

3.154

3.154

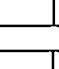

3.154

$d_{2}$

.147

.1961

.2389

.2739

\begin{tabular}{l|l}
2739 & .3041 \\
\hline
\end{tabular}

.3308

.3550

$L_{1}^{2}$

\begin{tabular}{|l|l}
\hline 211.7 & 211.7 \\
\hline
\end{tabular}

211.7

211.7

211.7

211.7

211.7

$L_{2}^{2}$

\begin{tabular}{|l|ll|l|l|l|l|l|}
\hline .01503 & .02697 & .04051 & .05382 & .06694 & .07989 & .09269 \\
\hline
\end{tabular}

$p$

.00002955

COMPOSITION EY WEIGHT FRACTION:

$$
\begin{array}{ll}
\text { Li } & .4645 \\
\mathrm{O} & .5355
\end{array}
$$

REFERENCE: 


\begin{tabular}{|c|c|c|c|c|c|c|c|}
\hline TEMPERATURE: & $60 \%$ & $500 \mathrm{~F}$ & $1000 \mathrm{~F}$ & $1500^{\circ} \mathrm{F}$ & $2000 \mathrm{~F}$ & $25000 \mathrm{~F}$ & $3000 \mathrm{~F}$ \\
\hline$D_{1}$ & .9493 & .9511 & .9524 & .9533 & .9540 & .9546 & .9550 \\
\hline$D_{2}$ & .8781 & .8891 & .8950 & .8984 & .9007 & .9022 & .9032 \\
\hline$\Sigma_{a}$ & .0002937 & .0002305 & .0001940 & .0001715 & .0001559 & .0001445 & .0001355 \\
\hline$\Sigma_{a_{2}}$ & .003000 & .002222 & .001803 & .001556 & .001384 & .001266 & .001170 \\
\hline$\Sigma_{s}$, & .001849 & .001970 & .002051 & .002108 & .002150 & .002185 & .002214 \\
\hline$d$ & 1.966 & 1.965 & 1.965 & 1.965 & 1. 964 & 1.964 & 1.963 \\
\hline$d_{1}$ & 2.023 & 2.027 & 2.030 & $2.032^{\circ}$ & 2.033 & 2.035 & 2.036 \\
\hline$d_{2}$ & 1.872 & 1.895 & 1.908 & 1.915 & 1.920 & 1.923 & 1.925 \\
\hline$L_{1}^{2}$ & 443.1 & 432.3 & 424.2 & 418.3 & 413.7 & 409.8 & 406.5 \\
\hline$L_{2}^{2}$ & 292.7 & 400.2 & 496.3 & 577.4 & 650.6 & 712.5 & 772.1 \\
\hline$p$ & .8629 & .8953 & .9136 & .9248 & .9324 & .9380 & .9423 \\
\hline
\end{tabular}

COMPOSITION OY WEIGHT FRACTION:

$\mathrm{Mg} .6032$

REFERENCE:

material: Magnesium

DENSITY VARIATIONS AND REFERENCE:

I. E. Campbell, High Temperature Technology, 1954 P. 31

$\mathrm{HCP}$

$3.58 \mathrm{gm} / \mathrm{cm}^{3}$

$3.58 \mathrm{gm} / \mathrm{cm}^{3}$

ASSUMEO DENSITY: $\quad 1,74 \mathrm{~g} / \mathrm{Cm}^{\circ}$

\begin{tabular}{|c|c|c|c|c|c|c|c|}
\hline TEMPERATURE: & $68 \mathrm{~F}$ & $500 \% \mathrm{~F}$ & $1000 \% \mathrm{~F}$ & $1500 \mathrm{~F}$ & $20000 \mathrm{~F}$ & $2500 \mathrm{~F}$ & $3000 \mathrm{~F}$ \\
\hline$D_{1}$ & 2.384 & 2.383 & 2.383 & 2.385 & 2.385 & 2.385 & 2.386 \\
\hline$D_{2}$ & 2.488 & 2. 408 & 2.376 & 2.362 & 2.354 & 2.350 & 2.347 \\
\hline$\Sigma_{a_{1}}$ & .0002197 & .0001761 & .0001502 & .0001346 & .0001233 & .0001148 & .0001081 \\
\hline$\Sigma_{a_{2}}$ & .002410 & .001785 & .001448 & .001250 & .001112 & .001017 & .0009397 \\
\hline$\Sigma_{s}$ & .0004827 & .0005424 & .0005812 & .0006064 & .0006258 & .0006412 & .0006540 \\
\hline$d$ & 5.118 & 5.091 & 5.075 & 5.067 & 5.060 & 5.055 & 5.052 \\
\hline$d_{1}$ & 5.081 & 5.079 & 5.079 & 5.083 & 5.084 & 5. 084 & 5.085 \\
\hline$d_{2}$ & 5.303 & 5.132 & 5.064 & 5.034 & 5.018 & 5.009 & 5.003 \\
\hline$L_{1}^{2}$ & 3394. & 3316. & 3258. & 3218. & 3184. & 3155. & 3131 . \\
\hline$L_{2}^{2}$ & 1032. & 1349 & 1640. & 1889. & 2117. & 2310. & 2498. \\
\hline$p$ & .6872 & .7549 & .7946 & .8183 & .8354 & .8482 & .8582 \\
\hline
\end{tabular}

COMPOSITION BY WEIGHT FRACTION:

Mg 1.00
DENSTTY YARIATIONS AND REFERENCE: HCP RH
$1.74 \mathrm{gm} / \mathrm{cm}^{3}$

$1.74 \mathrm{gm} / \mathrm{cm}^{3}$

REFERENCE: 


\begin{tabular}{|c|c|c|c|c|c|c|c|}
\hline TEMPERATURE: & $60 \%$ & $500 \mathrm{~F}$ & $1000 \mathrm{~F}$ & $15000 \mathrm{~F}$ & $20000 \%$ & $2500 \mathrm{~F}$ & $3000 \mathrm{~F}$ \\
\hline$D_{1}$ & .6804 & .6804 & .6804 & .6804 & .6804 & .6804 & .6804 \\
\hline$D_{2}$ & .2564 & .3013 & .3342 & .3578 & .3761 & .3912 & .4039 \\
\hline$\Sigma_{a_{1}}$ & .001194 & .001194 & .001193 & .001193 & .001193 & .001193 & .001193 \\
\hline$\Sigma_{a_{2}}$ & .8977 & .6693 & .5438 & .4697 & .4194 & .3825 & .3539 \\
\hline$\Sigma_{s_{1}}$ & 1. $214 \times 10^{-7}$ & $2.426 \times 10^{-7}$ & $3.693 \times 10^{-7}$ & $5.199 \times 10^{-7}$ & $6.636 \times 10^{-7}$ & $8.008 \times 10^{-7}$ & $9.341 \times 10^{-7}$ \\
\hline$d$ & 1.450 & 1. 450 & 1.450 & 1.450 & 1. 450 & 1.450 & 1.450 \\
\hline$d_{1}$ & 1. 450 & 1.450 & 1.450 & 1.450 & 1.450 & 1.450 & 1.450 \\
\hline$d_{2}$ & .5465 & .6423 & .7124 & .7626 & .8017 & .8337 & .8608 \\
\hline$L_{1}^{2}$ & 569.9 & 569.9 & 569.9 & 569.9 & 569.9 & 569.9 & 569.9 \\
\hline$L_{2}^{2}$ & .2856 & .4502 & .6147 & .7618 & .8967 & 1.023 & 1.141 \\
\hline$p$ & .0001017 & .0002032 . & .0003093 & .0004355 & .0005559 & .0006708 & .0007824 \\
\hline
\end{tabular}

COMPOSITION OY WEIGHT FRACTION:

$$
\begin{array}{llll}
W & .90 & \mathrm{Cu} & .04
\end{array}
$$

REFERENCE:
DOUSTYY VARIATIONS MMD REFEREMCE

P. R. Mallory

$16.8 \mathrm{gm} / \mathrm{cm}^{3}$

P. R. Mallory, Vendor

MATERIAL: Mercury
ASSUMED DENSITY: $\quad 13.55 \mathrm{~g} / \mathrm{Cm}^{\circ}$

\begin{tabular}{|c|c|c|c|c|c|c|c|}
\hline TEMPERATURE: & $\cos F$ & $500 \mathrm{FF}$ & $1000 \mathrm{~F}$ & $1500 \mathrm{~F}$ & $2000 \mathrm{~F}$ & $2500 \mathrm{~F}$ & $3000 \mathrm{~F}$ \\
\hline$D_{1}$ & 1.262 & 1.262 & 1.262 & 1.262 & 1,262 & 1.262 & 1.262 \\
\hline$D_{2}$ & .02255 & .02903 & .03506 & .04027 & .04490 & .04910 & .0 .5294 \\
\hline$\Sigma_{a_{1}}$ & .002737 & .002737 & .002737 & .002737 & .002737 & .002737 & .002737 \\
\hline$\Sigma_{a_{2}}$ & 13.97 & 10.67 & 8.696 & 7. 467 & 6.613 & 5.978 & 5.485 \\
\hline$\Sigma_{s_{1}}$ & $3.078 \times 10^{-13}$ & $6.244 \times 10^{-13}$ & $9.571 \times 10^{-13}$ & $1.365 \times 10^{-12}$ & $1.756 \times 10^{-12}$ & $2.132 \times 10^{-12}$ & $2.497 \times 10^{-12}$ \\
\hline$d$ & 2.689 & 2.689 & 2.689 & 2.689 & 2.689 & 2.689 & 2.689 \\
\hline$d_{1}$ & 2.689 & 2.689 & 2.689 & 2.689 & 2.689 & 2.689 & 2.689 \\
\hline$d_{2}$ & .04807 & .06189 & .07473 - & .08583 & .09571 & .1047 & .1128 \\
\hline$L_{1}^{2}$ & 461.0 & 461.0 & 461.0 & 461.0 & 461.0 & 461.0 & 461.0 \\
\hline$L_{2}^{2}$ & .001614 & .002721 & .004032 & .005392 & .006790 & .008214 & .009651 \\
\hline$p$ & $1.125 \times 10^{-10}$ & $2.282 \times 10^{-10}$ & $3.497 \times 10^{-10}$ & $4.988 \times 10^{-10}$ & $6.418 \times 10^{-10}$ & $7.789 \times 10^{-10}$ & $9.126 \times 10^{-10}$ \\
\hline
\end{tabular}

COMPOSITION GY WEIGHT FRACTION:

Hg 1.00
DENSITY VARIATIONS AND REFERENCE:

HCP

$\mathrm{RH}$
$13.546 \mathrm{gm} / \mathrm{cm}^{3}$

$13.55 \mathrm{gm} / \mathrm{cm}^{3}$

REFERENCE: 


\begin{tabular}{|c|c|c|c|c|c|c|c|}
\hline TEMPERATURE: & $\cos$ & $500 \mathrm{~F}$ & $1000 \mathrm{~F}$ & $1500 \mathrm{~F}$ & $2000 \mathrm{~F}$ & $25000 \mathrm{~F}$ & $3000^{\circ} \mathrm{F}$ \\
\hline$D_{1}$ & .9544 & .9545 & .9546 & .9548 & '. .9549 & .9550 & .9552 \\
\hline$D_{2}$ & .3084 & .3112 & .3227 & .3281 & .3318 & .3340 & .3356 \\
\hline$\Sigma_{a_{1}}$ & .001456 & .001431 & .001407 & .001386 & .001367 & .001350 & .001334 \\
\hline$\Sigma_{a_{2}}$ & .1876 & .1391 & .1128 & .09737 & .08691 & .07923 & .07328 \\
\hline$\Sigma_{s_{1}}$ & .00003642 & .00006153 & .00008554 & .0001073 & .0001265 & .0001440 & .0001601 \\
\hline$d$ & 2.034 & 2.034 & 2.034 & 2.034 & 2.033 & 2.033 & 2.033 \\
\hline$d_{1}$ & 2.034 & 2.034 & 2.035 & 2.035 & 2.035 & 2.036 & 2.036 \\
\hline$d_{2}$ & .6573 & .6632 & .6878 & .6992 & .7071 & .7119 & .7153 \\
\hline$L \frac{2}{2}$. & 639.5 & 639.4 & 639.4 & 639.3 & 639.3 & 639.2 & 639.2 \\
\hline$L \frac{2}{2}$ & 1.643 & 2.236 & 2.860 & 3.369 & 3.817 & 4.216 & 4.579 \\
\hline$p$ & .02440 & .04122 & .05730 & .07183 & .08469 & .09638 & .1071 \\
\hline
\end{tabular}

COMPOSITION BY WEIGHT FRACTION:

$$
\text { Fe } .99
$$$$
\text { C } .01
$$

HCP
DENSITY VARIATIONS AND REFERENCE: HCP

$7.83 \mathrm{gm} / \mathrm{cm}^{3}$

\begin{tabular}{|c|c|c|c|c|c|c|c|}
\hline TEMPERATURE: & $68 \% F$ & $500 \% \mathrm{~F}$ & $1000 \mathrm{~F}$ & $15000 \%$ & $20000 \mathrm{~F}$ & $2500 \mathrm{~F}$ & $3000 \mathrm{~F}$ \\
\hline$D_{1}$ & .8639 & .8639 & .8639 & .8639 & .8639 & .8639 & .8639 \\
\hline$D_{2}$ & .7018 & .7304 & .7478 & .7595 & .7684 & .7755 & .7818 \\
\hline$\Sigma_{a_{1}}$ & .001760 & .001760 & .001760 & .001760 & .001760 & .001760 & .001760 \\
\hline$\Sigma_{a_{2}}$ & .1420 & .1053 & .08538 & .07369 & .06578 & .05996 & .05546 \\
\hline$\Sigma_{s_{2}}$ & $3.000 \times 10^{-9}$ & $5.669 \times 10^{-9}$ & $8.398 \times 10^{-9}$ & $1.130 \times 10^{-8}$ & $1.402 \times 10^{-8}$ & $1.659 \times 10^{-8}$ & $1.904 \times 10^{-8}$ \\
\hline$d$ & 1.842 & 1.842 & 1.842 & 1. 842 & 1.842 & 1.842 & 1.842 \\
\hline$d_{1}$ & 1.842 & 1.842 & $1.8 \underline{2}$ & 1.842 & 1.842 & 1.842 & 1.842 \\
\hline$d_{2}$ & 1.496 & 1.557 & 1.594 & 1.619 & 1.638 & 1.653 & 1.666 \\
\hline$L \frac{\dot{z}}{2}$ & 490.8 & 490.8 & $490^{\circ} .8$ & 490.8 & 490.8 & 490.8 & 490.8 \\
\hline & 2 & & & & $\therefore$ & & \\
\hline$L \frac{2}{2}$ & 4.942 & 6.936 & 8.759 & 10.31 & 11.68 & 12.93 & 14.10 \\
\hline$p$ & $1.704 \times 10^{-6}$ & $3.221 \times 10^{-6}$ & $4.771 \times 10^{-6}$ & $6.418 \times 10^{-6}$ & $7.965 \times 10^{-6}$ & $9.422 \times 10^{-6}$ & $1.081 \times 10^{-5}$ \\
\hline
\end{tabular}

COMPOSITION QY WEIGHT FRACTION:

$$
\text { Mo } 1.00
$$

REFERENCE
DENSITY VARIATIONS AND REFERENCE: ANPD: E. A. Aitken $10.2 \mathrm{gm} / \mathrm{cm}^{3}$ 


\begin{tabular}{|c|c|c|c|c|c|c|c|}
\hline TEMPERATURE: & cose & 500\% & $1000 \mathrm{FF}$ & $1500 \mathrm{~F}$ & $20000 \mathrm{~F}$ & $25000 \mathrm{Fr}$ & $3000 \mathrm{~F}$ \\
\hline$D_{1}$ & .7458 & .7458 & .7459 & .7460 & .7461 & .7462 & 1.7462 \\
\hline$D_{2}$ & .1762 & .1824 & .1863 & .1888 & .1907 & .1922 & .1935 \\
\hline$\Sigma_{a_{1}}$ & .002111 & .002082 & .002055 & $.002,029$ & .002006 & .001985 & .001966 \\
\hline$\Sigma_{a_{2}}$ & .3728 & .2764 & .2241 & .1935 & $.1727^{\circ}$ & .1574 & .1456 \\
\hline$\Sigma_{s_{1}}$ & .00003810 & .00006676 & .00009482 & .0001209 & .0001440 & .0001652 & .0001850 \\
\hline$d$ & 1.590 & 1.589 & 1.589 & 1.589 & 1.589 & 1.589 & 1.589 \\
\hline$d_{1}$ & 1.590 & 1.590 & 1.590 & 1.590 & 1.590 & 1.590 & 1.591 \\
\hline$a_{2}$ & .3755 & .3888 & .3970 & .4024 & .4065 & .4098 & .4125 \\
\hline$L_{1}^{2}$ & 347.1 & 347.1 & 347.0 & 347.0 & 347.0 & 347.0 & 347.0 \\
\hline$L_{2}^{2}$ & .4726 & .6598 & .8310 & .9760 & 1.104 & 1.221 & 1.329 \\
\hline$p$ & .01773 & .03107 & .04411 & .05623 & .06698 & .07685 & .08602 \\
\hline \multicolumn{6}{|c|}{ COMPOSITION QY WEIGHT FRACTION: } & \multicolumn{2}{|c|}{ DENSITY VARIATIONS AND REFERENCI } \\
\hline 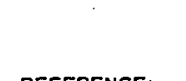 & $\mathrm{Ni} 1.00$ & & & & & & $\begin{array}{l}3-8.90 \mathrm{gm} / \mathrm{cm} \\
22 \mathrm{gm} / \mathrm{cm}^{3}\end{array}$ \\
\hline
\end{tabular}

REFERENCE:

\begin{tabular}{|c|c|c|c|c|c|c|c|}
\hline TEMPERATURE: & off & soor F & $1000 \mathrm{FF}$ & $15000 \mathrm{~F}$ & $2000 \mathrm{FF}$ & $2500 \mathrm{~F}$ & $3000 \mathrm{~F}$ \\
\hline$D_{2}$ & .9368 & .9368 & .9368 & .9368 & 9368 & .9368 & .9368 \\
\hline$D_{2}$ & .9402 & .9443 & .9450 & .9451 & .9451 & .9450 & .9448 \\
\hline$\Sigma_{a_{1}}$ & .0006878 & .0006799 & .0006721 & .0006649 & .0006584 & .0006523 & .0006467 \\
\hline$\Sigma_{a_{2}}$ & .05422 & .04020 & .03260 & .02813 & .02511 & .02289 & .02117 \\
\hline$\dot{\Sigma}_{s_{1}}$ & .00001057 & .00001862 & .00002655 & .00003399 & .00004075 & .00004698 & .00005280 \\
\hline$d$ & 1.997 & 1.997 & 1.997 & 1.997 & 1.997 & 1.997 & 1.997 \\
\hline$d_{2}$ & 1.997 & 1.997 & 1.997 & 1.997 & 1.997 & 1.997 & 1.997 \\
\hline$d_{2}$ & 2.004 & 2.013 & 2.014 & 2.015 & 2.014 & 2.014 & 2.014 \\
\hline$L_{1}^{2}$ & 1341. & 1341. & 1341. & 1340. & 1340. & 1340. & 1339. \\
\hline$L_{\frac{2}{2}}$ & 17.34 & 23.49 & 28.99 & 33.00 & 07.03 & 11,28 & $44.6 ?$ \\
\hline$p$ & .01514 & .02665 & .03800 & .04863 & .05829 & .06718 & .07548 \\
\hline
\end{tabular}

COMPOSITION BY WEIGHT FRACTION:

$\mathrm{Nb} 1.00$

REFERENCE:
DENSITY VARIATIONS AND REFERENCE:

ANPD: E. A. Aitken 8.57 or $8.4 \mathrm{gm} / \mathrm{cm}^{3}$

HCP $\quad 8.4 \mathrm{gm} / \mathrm{cm}^{3}$

RH $\quad 8.57 \mathrm{gm} / \mathrm{cm}^{3}$ 


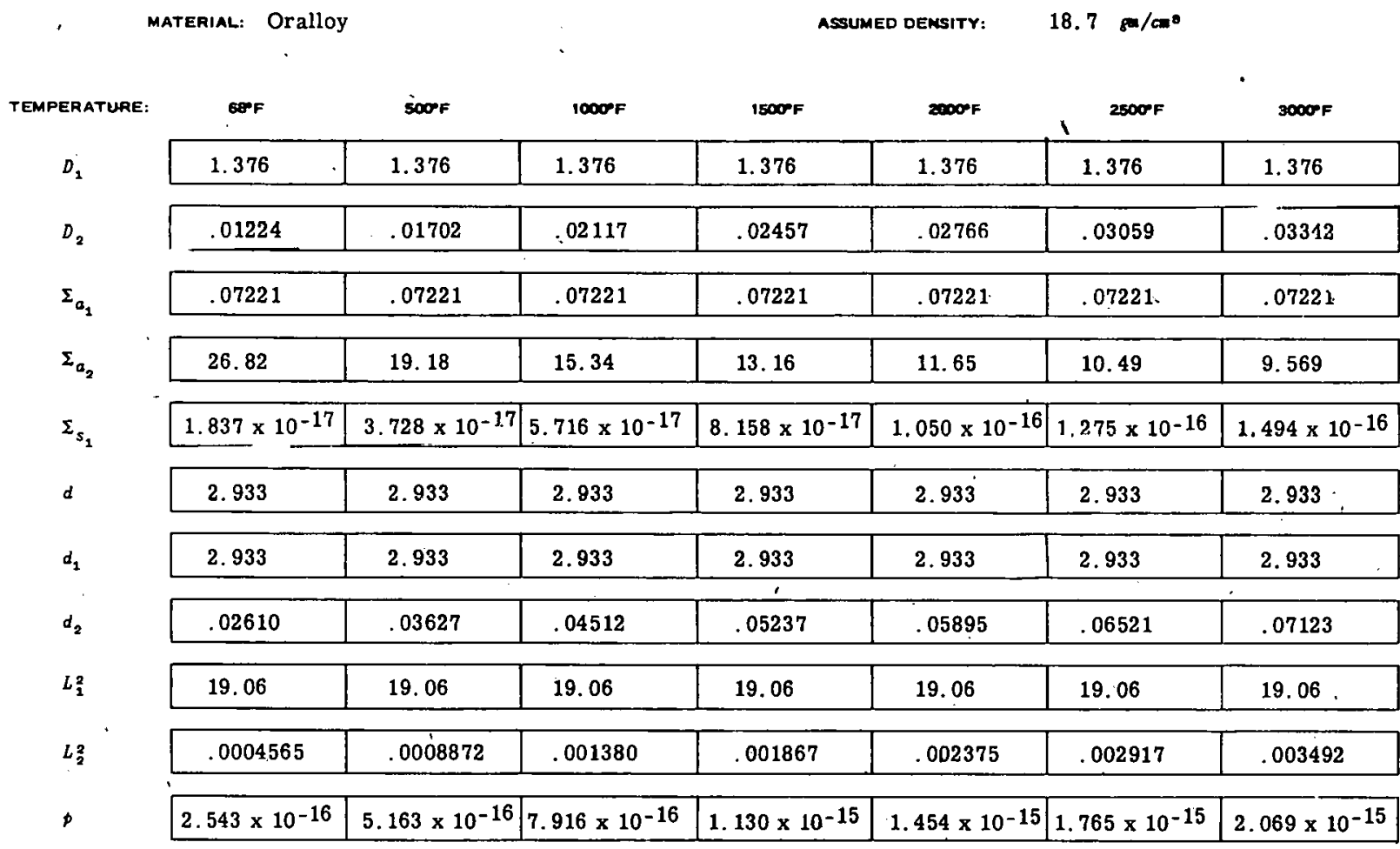

COMPOSITION BY WEIGHT FRACTION:

REFERENCE:

$$
\begin{array}{cc}
U^{238} & .068 \\
U^{235} & .932
\end{array}
$$

DENSITY YARIATIONS AND REFERENCE:

ANPD: J. D. Simpson

$18.7 \mathrm{gm} / \mathrm{cm}^{3}$

for ingot

mATERIAL: Oralloy

\begin{tabular}{|c|c|c|c|c|c|c|c|}
\hline TEMPERATURE: & 6ேF & $500 \mathrm{~F}$ & $1000 \mathrm{~F}$ & $1500 \mathrm{~F}$ & $2000^{\circ} \mathrm{F}$ & $2500^{\circ} \mathrm{F}$ & $3000 \mathrm{~F}$ \\
\hline $\boldsymbol{v} \mathbf{\Sigma}_{f_{1}}$ & .1452 & .1452 & .1452 & .1452 & .1452 & .1452 & .1452 \\
\hline$v \Sigma_{f_{2}}$ & 55.24 & 39.45 & 31.38 & 26.80 & 23.66 & 21.30 & 19.43 \\
\hline$k_{1}$ & 2.011 & 2.011 & 2.011 & 2. 011 & 2.011 & 2.011 & 2.011 \\
\hline$k$ & 2.060 & 2.056 & 2.046 & 2.037 & 2.032 & 2.030 & 2.030 \\
\hline
\end{tabular}




\begin{tabular}{|c|c|c|c|c|c|c|c|}
\hline TEMPERATURE: & $1^{60 \% F}$ & $1^{500 \% F}$ & $1000 \% \mathrm{~F}$ & $1500 \mathrm{~F}$ & $2000 \mathrm{~F}$ & $2500^{\circ} \mathrm{F}$ & $3000 \mathrm{FF}$ \\
\hline$=D_{1}$ & 1.354 & 1.354 & 1.354 & 1.354 & 1.354 & 1.354 & 1.354 \\
\hline$D_{2}$ & .02340 & .03238 & .04012 & .04642 & .05210 & .05747 & 06260 \\
\hline$\Sigma_{a_{1}}$ & $.03908^{\prime}$ & .03908 & .03908 & .03908 & .03908 & .03908 & .03908 \\
\hline$\Sigma_{a_{2}}$ & 13.84 & 9.897 & 7.915 & 6.790 & 6.009 & 5.412 & 4.937 \\
\hline$' \Sigma_{s_{1}}$ & $5.709 \times 10^{-12}$ & $1.155 \times 10^{-11}$ & $1.769 \times 10^{-11}$ & $2.520 \times 10^{-11}$ & $3.239 \times 10^{-11}$ & $3.929 \times 10^{-11}$ & $4.601 \times 10^{-11}$ \\
\hline$d$ & 2.886 & 2.886 & 2.886 & 2,886 & 2.886 & 2.886 & 2.886 \\
\hline$d_{1}$ & 2.886 & 2.886 & 2.886 & 2.886 & 2.886 & 2.886 & 2.886 \\
\hline$d_{2}$ & .04988 & .06902 & .08552 & .09894 & .1110 & .1225 & 1334 \\
\hline$L_{1}^{2}$ & 34.64 & 34.64 & 34.64 & 34.64 & 34.64 & 34,64 & 34.64 \\
\hline$L \frac{2}{2}$ & .001691 & .003272 & .005069 & .006836 & .008670 & .01062 & .01268 \\
\hline$p$ & $1.461 \times 10^{-10}$ & $2.956 \times 10^{-10}$ & $4.527 \times 10^{-10}$ & $6.447 \times 10^{-10}$ & $8.288 \times 10^{-10}$ & $1.005 \times 10^{-9}$ & $1.177 \times 10^{-9}$ \\
\hline \multicolumn{6}{|c|}{ COMPOSITION BY WEIGHT FRACTION: } & \multicolumn{2}{|c|}{ DENSITY VARIATIONS AND REFERENCE: } \\
\hline & $\begin{array}{lll}\mathrm{U}^{235} & .819 \\
\mathrm{U}^{238} & .061\end{array}$ & .120 & & & A & ANPD; E. A. A & $\begin{array}{l}\text { itken } \\
10.97 \mathrm{gm} / \mathrm{cm}^{3}\end{array}$ \\
\hline
\end{tabular}

material: Oralloy Dioxide

\begin{tabular}{|c|c|c|c|c|c|c|c|}
\hline \multirow{2}{*}{ TEMPERATURE: } & \multirow[b]{2}{*}{$60^{\circ} \mathrm{F}$} & \multirow{2}{*}{$5000 \mathrm{~F}$} & \multirow{2}{*}{$1000 \mathrm{~F}$} & \multirow{2}{*}{$1500 \mathrm{~F}^{\circ}$} & \\
\hline & & & & & $2000^{\circ} \mathrm{F}$ & $2500^{\circ} \mathrm{F}$ & $30000^{\circ} \mathrm{F}$ \\
\hline$\nu \Sigma_{f_{1}}$ & .07848 & .07848 & .07848 & .07848 & .07848 & .07848 & .07848 \\
\hline$\nu \Sigma_{f_{2}}$ & 28.50 & 20.35 & 16.19 & 13.83 & $\mathbf{i 2 . 2 1}$ & 10.99 & 10.02 \\
\hline$k_{2}$ & 2.008 & 2,008 & 2.008 & 2.008 & 2.008 & 2.008 & 2.008 \\
\hline$k_{2}$ & 2.060 & 2.056 & 2.046 & 2.037 & 2.032 & 2.030 & 2.030 \\
\hline
\end{tabular}




\begin{tabular}{|c|c|c|c|c|c|c|c|}
\hline TEMPERATURE: & $60 \%$ & $500 \mathrm{~F}$ & $1000{ }^{\circ} \mathrm{F}$ & $1500 \mathrm{~F}^{\circ}$ & $2000 \mathrm{FF}$ & $2500 \mathrm{~F}$ & $3000 \mathrm{~F}$ \\
\hline$D_{1}$ & 1.214 & 1.232 & 1.244 & 1.253 & 1.260 & 1.266 & 1.270 \\
\hline$D_{2}$ & .1624 & .2080 & .2472 & .2781 & .3037 & .3254 & .3441 \\
\hline$z_{a_{1}}$ & .001041 & .0007927 & .0006586 & .0005777 & .0005224 & .0004817 & .0004500 \\
\hline$\Sigma_{a_{2}}$ & .01693 & .01255 & .0 .10 .18 & .008784 & .007841 & .007147 & .006610 \\
\hline$\Sigma_{s_{1}}$ & .03798 & .03892 & .03955 & .04000 & .04035 & .04063 & .04087 \\
\hline$d$ & 1.037 & .9754 & .9618 & .9669 & .9790 & .9933 & 1.008 \\
\hline$d_{1}$ & 2,587 & 2.625 & 2.652 & 2.671 & 2.686 & 2.698 & 2.708 \\
\hline$d_{2}$ & .3462 & .4433 & .5268 & .5927 & .6473 & .6935 & .7334 \\
\hline$L_{1}^{2}$ & 31.10 & 31.02 & 30.95 & 30.88 & 30.83 & 30.79 & 30.74 \\
\hline$L_{2}^{2}$ & 9. 596 & 16.58 & 24.29 & 31.66 & 38.73 & 45.52 & 52.06 \\
\hline$p$ & .9733 & .9800 & .9836 & .9858 & .9872 & .9883 & .9891 \\
\hline
\end{tabular}

COMPOSITION EY WEIGHT FRACTION:

REFERENCE:
DENSITY VARIATIONS AND REFERENCE: $\mathrm{HCP} 1.18 \mathrm{gm} / \mathrm{cm}^{3}$

$$
\begin{array}{llll}
\text { C } & .587 & \text { O } & .3315
\end{array}
$$

ORNL-1615, p. 94

MATERIAL: Plutonium 239
ASSLMED DENSITY: $\quad 19.00 \mathrm{gm} / \mathrm{cm}$

\begin{tabular}{|c|c|c|c|c|c|c|c|}
\hline TEMPERATURE: & 605 & $5000 \mathrm{~F}$ & $1000 \mathrm{~F}$ & $1500 \mathrm{FF}$ & $2000 \mathrm{~F}$ & $2500 \mathrm{~F}$ & 3nopor \\
\hline$D_{1}$ & .9136 & .9136 & .9136 & .9136 & .9136 & .9136 & .9136 \\
\hline$D_{2}$ & .006890 & .006921 & .005697 & .005026 & .004827 & .004890 & .005108 \\
\hline$\Sigma_{a_{1}}$ & .1269 & .1269 & .1269 & .1269 & .1269 & .1269 & .1269 \\
\hline$\Sigma_{a_{2}}$ & 47.90 & 47.69 & 58.05 & 65.87 & 68.63 & 67.75 & 64.84 \\
\hline$\Sigma_{s_{1}}$ & $2.047 \times 10^{-17}$ & $4.156 \times 10^{-17}$ & $6.373 \times 10^{-17}$ & $9.097 \times 10^{-17}$ & $1.171 \times 10^{-16}$ & $1.422 \times 10^{-16}$ & $1.666 \times 10^{-16}$ \\
\hline$d$ & 1.947 & 1.947 & 1.947 & 1.947 & 1.947 & 1.947 & 1.947 \\
\hline$d_{2}$ & 1.947 & 1.947 & 1.947 & 1.947 & 1.947 & 1.947 & 1.947 \\
\hline$d_{2}$ & .01469 & .01475 & .01214 & .01071 & .01029 & .01042 & .01089 \\
\hline$L_{1}^{2}$ & 7.198 & 7. 198 & 7.198 & 7.198 & 7.198 & 7.198 & 7. 198 \\
\hline$L_{2}^{2}$ & .0001438 & .0001451 & 1.00009815 & .00007630 & .00007033 & .00007218 & .00007878 \\
\hline$p$ & $1.613 \times 10^{-16}$ & $3.275 \times 10^{-16}$ & $5.022 \times 10^{-16}$ & $7.168 \times 10^{-16}$ & $9.228 \times 10^{-16}$ & $1.120 \times 10^{-15}$ & $1.313 \times 10^{-15}$ \\
\hline
\end{tabular}

COMPOSITION OY WEIGHT FRACTION:

$\mathrm{Pu}^{239} 1.00$

REFERENCE:
DENSITY VARIATIONS AND REFERENCE:

W., B. H. Lord, Nature 173, $534-5$, (1954) $19.0 \mathrm{gm} / \mathrm{cm}^{3}$ 


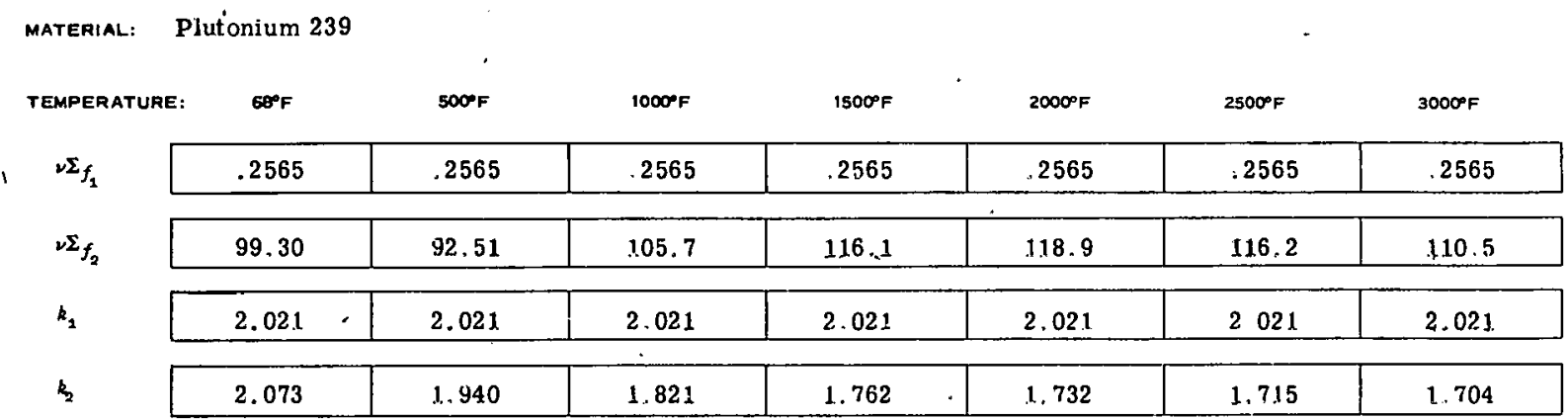

material: Potassium
ASSUMEO DENSITY: $\quad .870 \quad \mathrm{~m}^{\circ}$

\begin{tabular}{|c|c|c|c|c|c|c|c|}
\hline \multirow[b]{2}{*}{ TEMPERATURE: } & \multirow{2}{*}{\multicolumn{2}{|c|}{ TERIAL: Potassium }} & \multirow[b]{2}{*}{$1000 \mathrm{~F}$} & \multicolumn{2}{|c|}{ ASSUMEO DENSITY: } & \multicolumn{2}{|l|}{$.870 \mathrm{gm} / \mathrm{cm}^{\circ}$} \\
\hline & & & & $1500 \mathrm{~F}$ & $2000 \mathrm{~F}$ & $2500 \% F$ & $3000 \mathrm{~F}$ \\
\hline \multirow{2}{*}{\multicolumn{8}{|c|}{$\begin{array}{l}14.31 \\
1 \\
\end{array}$}} \\
\hline & & & & & & & \\
\hline$D_{2}$ & 6.265 & 7.172 & 7.796 & 8.224 & 8.546 & 8.801 & 9.009 \\
\hline$\Sigma_{a_{1}}$ & .0001302 & .0001299 & .0001296 & .0001293 & .0001290 & .0001287 & .0001285 \\
\hline$\Sigma_{a_{2}}$ & .02342 & .01736 & .01408 & .01215 & .01084 & .009886 & .009144 \\
\hline$\Sigma_{s_{1}}$ & $3.306 \times 10^{-7}$ & $6.186 \times 10^{-7}$ & $9.116 \times 10^{-7}$ & $1.222 \times 10^{-6}$ & $1.512 \times 10^{-6}$ & $1.785 \times 10^{-6}$ & $2.045 \times 10^{-6}$ \\
\hline$d$ & 30.51 & 30.51 & 30.51 & 30.51 & 30.51 & 30.51 & 30.51 \\
\hline$d_{1}$ & 30.51 & 30.51 & 30.51 . & 30.51 & 30.51 & 30.51 & 30.51 \\
\hline$d_{2}$ & 13.35 & 15.29 & 16.62 & 17.53 & 18.22 & 18.76 & 19.20 \\
\hline$L_{1}^{2}$ & 109700. & 109700. & 109700. & 109700. & 109700 & 109700. & 109700. \\
\hline$L_{2}^{2}$ & 267.6 & 413.1 & 553.8 & 676.9 & 788.1 & 890.2 & 985.3 \\
\hline$p$ & .002533 & .004740 & .006985 & .009363 & .01158 & .01367 & .01567 \\
\hline COMPOSITION BY & VEIGHT FRACTIO & & & & & DENSITY VARIA & S AND REFERENCE: \\
\hline & $\mathrm{K} 1.00$ & & & & & $\mathrm{HCP}$ & $.87 \mathrm{gm} / \mathrm{cm}^{3}$ \\
\hline
\end{tabular}

REFERENCE: 


\begin{tabular}{|c|c|c|c|c|c|c|c|}
\hline TEMPERATURE: & $60 \%$ & $300 \% \mathrm{~F}$ & $1000 \mathrm{~F}$ & $1500 \mathrm{~F}$ & $2000 \mathrm{~F}$ & $2500 \mathrm{~F}$ & $3000^{\circ} \mathrm{F}$ \\
\hline$D_{1}$ & 16.93 & 16.94 & 16.95 & 16.95 & 16.96 & $16.96^{\circ}$ & 16.97 \\
\hline$D_{2}$ & 12.08 & 13.10 & 13.69 & 14.05 & 14.30 & 14.47 & 14.61 \\
\hline$\Sigma_{a_{1}}$ & .0001455 & .0001376 & .0001308 & .0001256 & .0001213 & .0001176 & .0001143 \\
\hline$\Sigma_{a_{2}}$ & .005028 & .003728 & .003023 & .002609 & .002329 & .002123 & .001964 \\
\hline$\Sigma_{s,}$ & .00001771 & .00002593 & .00003311 & .00003868 & .00004331 & .00004733 & .00005088 \\
\hline$d$ & 36.05 & 36.05 & 36.05 & 36.04 & $=36.04$ & 36.04 & 36.04 \\
\hline$d_{1}$ & 36.09 & 36.11 & 36.12 & 36.14 & 36.15 & 36.16 & 36.17 \\
\hline$d_{2}$ & 25.74 & 27.92 & 29.19 & 29.95 & 30.47 & 30.85 & 31.14 \\
\hline & & & & & - & & \\
\hline$L_{1}^{2}$ & 103700 & 103600 & 103400 & 103200 & 103000. & 102900. & 102700 \\
\hline$L_{2}^{2}$ & 2402. & 3513. & 4530 . & 5386. & 6138. & 6817. & 7439. \\
\hline$p$ & .1085 & .1586 & .2020 & .2354 & .2632 & .2870 & .3080 \\
\hline
\end{tabular}

COMPOSITION BY WEIGKT FRACTION:

REFERENCE:

\begin{tabular}{|c|c|c|c|c|c|c|c|}
\hline TEMPERATURE: & $\cos$ & $5000 \mathrm{~F}$ & $1000 \mathrm{~F}$ & $1500 \mathrm{~F}$ & $2000-F$ & $2500 \mathrm{~F}$ & $3000 \mathrm{~F}$ \\
\hline$D_{1}$ & 1.108 & 1. 108 & 1.108 & 1.108 & 1. 108 & 1.108 & 1.108 \\
\hline$D_{2}$ & .001397 & .001393 & .001720 & .002203 & .002793 & .003470 & .004223 \\
\hline$\Sigma_{a_{1}}$ & .03782 & .03782 & .03782 & .03782 & .03782 & .03782 & .03782 \\
\hline$\Sigma_{a_{2}}$ & 236.9 & 237.3 & 192.0 & 149.8 & 118.0 & 94.91 & . 77.91 \\
\hline$\Sigma_{s_{1}}$ & $7.792 \times 10^{-19}$ & $1.582 \times 10^{-18}$ & $2.426 \times 10^{-18}$ & $3.462 \times 10^{-18}$ & $4.456 \times 10^{-18}$ & $5.409 \times 10^{-18}$ & $6.339 \times 10^{-18}$ \\
\hline$d$ & 2.362 & 2.362 & 2.362 & 2.362 & 2.362 & 2. 362 & 2.362 \\
\hline$d_{1}$ & 2.362 & 2.362 & 2.362 & 2.362 & 2.362 & 2. 362 & 2.362 \\
\hline$d_{2}$ & .002979 & .002969 & .003666 & .004696 & .005953 & . 007397 & .009002 \\
\hline$L_{1}^{2}$ & 29.30 & 29.30 & 29.30 & 29.30 & 29.30 & 29.30 & 29.30 \\
\hline$L_{2}^{2}$ & $5.899 \times 10^{-6}$ & $5.871 \times 10^{-6}$ & $8.959 \times 10^{-6}$ & .00001471 & .00002366 & .00003656 & .00005421 \\
\hline$p$ & $2.060 \times 10^{-17}$ & $4.182 \times 10^{-17}$ & $6.413 \times 10^{-17}$ & $9.153 \times 10^{-17}$ & $1.178 \times 10^{-16}$ & $1.430 \times 10^{-16}$ & $1.676 \times 10^{-16}$ \\
\hline
\end{tabular}

COMPOSITION BY WEIGHT FRACTION:

Sm 1.00
DENSITY VARIATIONS AND REFERENCE: ANPD: E. A. Aitken

$7.54 \mathrm{gm} / \mathrm{cm}^{3}$ 


\begin{tabular}{|c|c|c|c|c|c|c|c|}
\hline TEMPERATURE: & 6orF & $500 \mathrm{~F}$ & $1000 \mathrm{~F}$ & $1500 \mathrm{~F}$ & $2000 \mathrm{~F}$ & $2500 \mathrm{~F}$ & $3000 \mathrm{~F}$ \\
\hline$D_{1}$ & .9776 & .9776 & .9776 & .9776 & .9776 & .9776 & .9776 \\
\hline$D_{2}$ & .001643 & .001638 & .002022 & .002589 & .003282 & .004077 & .004959 \\
\hline$\Sigma_{a}$ & .03535 & .03535 & .03535 & .03535 & .03535 & .03535 & .03535 \\
\hline$\Sigma_{a_{2}}$ & 201.3 & 201.7 & 163.2 & 127.3 & 100.3 & 80.66 & 66.21 \\
\hline$\Sigma_{s_{1}}$ & $3.570 \times 10^{-14}$ & $7.246 \times 10^{-14}$ & $1.111 \times 10^{-13}$ & $1.585 \times 10^{-13}$ & $2.039 \times 10^{-13}$ & $2.475 \times 10^{-13}$ & $2.900 \times 10^{-13}$ \\
\hline$d$ & 2.084 & 2.084 & 2.084 & 2.084 & 2.084 & 2.084 & 2.084 \\
\hline$d_{1}$ & 2.084 & 2.084 & 2.084 & 2.084 & 2.084 & 2.084 & 2.084 \\
\hline$d_{2}$ & .003502 & .003492 & .004310 & .005519 & .006995 & .008689 & .01057 \\
\hline & & & & & & 1 & \\
\hline$L_{1}^{2}$ & 27.65 & 27.65 & 27.65 & 27.65 & 27.65 & 27.65 & 27.65 \\
\hline$L_{2}^{2}$ & $8.162 \times 10^{-6}$ & $8.123 \times 10^{-6}$ & $1.239 \times 10^{-5}$ & $2.034 \times 10^{-5}$ & $3.271 \times 10^{-5}$ & $5.054 \times 10^{-5}$ & $7.491 \times 10^{-5}$ \\
\hline$p$ & $1.010 \times 10^{-12}$ & $2.050 \times 10^{-12}$ & $3.143 \times 10^{-12}$ & $4.483 \times 10^{-12}$ & $5.769 \times 10^{-12}$ & $7.001 \times 10^{-12}$ & $8.203 \times 10^{-12}$ \\
\hline
\end{tabular}

COMPOSITION BY WEIGHT FRACTION:

REFERENCE:
Sm .8624

O .1376
DENSITY VARIATIONS AND REFEREMCE

ANPD: E. A. Aitken

$7.43 \mathrm{gm} / \mathrm{cm}^{3}$
MATERIALI Scandium
ASSUMED DENSITY: $\quad 3.200 \mathrm{~m} / \mathrm{cm}^{*}$

\begin{tabular}{|c|c|c|c|c|c|c|c|}
\hline TEMPERATURE: & $\infty \mathrm{F}$ & $800 \mathrm{~F}$ & $1000 \mathrm{~F}$ & $1500 \% \mathrm{~F}$ & $2000 \mathrm{Fr}$ & $2500 \mathrm{~F}$ & S000\% F \\
\hline$D_{1}$ & 2.583 & 2.583 & 2.583 & 2.583 & 2.583 & 2.583 & 2.583 \\
\hline$D_{2}$ & .1656 & .1906 & .2076 & .2191 & .2277 & .2345 & .2401 \\
\hline$\Sigma_{a_{1}}$ & .001039 & .001038 & .001038 & .001038 & .001037 & .001037 & .001036 \\
\hline$\Sigma_{a_{2}}$ & .9125 & .6778 & .5501 & .4746 & .4230 & .3848 & .3550 \\
\hline$\Sigma_{s_{1}}$ & $4.310 \times 10^{-7}$ & $8.175 \times 10^{-7}$ & $1.213 \times 10^{-6}$ & $1.642 \times 10^{-6}$ & $2.044 \times 10^{-6}$ & $2.423 \times 10^{-6}$ & $2.787 \times 10^{-6}$ \\
\hline$d$ & 5.505 & 5.505 & 5.505 & 5.505 & 5.505 & 5.505 & 5.505 \\
\hline$d_{1}$ & 5.505 & 5.505 & 5.505 & 5.505 & 5.505 & 5.505 & 5.505 \\
\hline$d_{8}$ & .3530 & .4063 & .4425 & .4670 & .4853 & .4998 & .5117 \\
\hline$L_{1}^{2}$ & 2485. & 2485. & 2485. & 2485. & 2485 . & 2485. & 2485 . \\
\hline$L \frac{2}{2}$ & .1815 & .2812 & .3774 & .4617 & .5382 & .6093 & .6762 \\
\hline$p$ & .0004147 & .0007867 & .001167 & .001580 & .001967 & .002332 & .002682 \\
\hline
\end{tabular}

COMPOSITION BY WEIGHT FRACTION:

Sc 1.00

REFERENCE:
DENSITY VARIATIONS AND REFERENCE

ANPD: C. R. Simmons

$3.2 \pm .1 \mathrm{gm} / \mathrm{cm}^{3}$ (experimental) 


\begin{tabular}{|c|c|c|c|c|c|c|c|}
\hline TEMPERATURE: & $\cos F$ & $500 \mathrm{~F}$ & $1000 \mathrm{~F}$ & $15000 \mathrm{~F}$ & $2000 \mathrm{~F}$ & $2500 \mathrm{~F}$ & $3000 \mathrm{~F}$ \\
\hline$D_{1}$ & 3.181 & 3.183 & 3. 184 & 3.186 & 3.186 & 3.187 & 3.187 \\
\hline$D_{2}$ & 3.053 & 3.095 & 3.119 & 3.133 & 3.142 & 3.149 & 3.155 \\
\hline$\Sigma_{a_{1}}$ & .0003301 & .0002900 & .0002604 & .0002414 & .0002264 & .0002144 & .0002045 \\
\hline$\Sigma_{a_{2}}$ & .005761 & .004272 & .003464 & .002990 & .002669 & .002433 & .002250 \\
\hline$\Sigma_{s_{1}}$ & .0001643 & .0002090 & .0002427 & .0002651 & .0002832 & .0002979 & .0003103 \\
\hline$d$ & 6.773 & 6.775 & 6,777 & 6.781 & 6.783 & G. 784 & 6.785 \\
\hline$d_{1}$ & 6.781 & 6.784 & 6.786 & 6.790 & 6.792 & 6.793 & 6.794 \\
\hline$d_{2}$ & 6.508 & 6.598 & 6.648 & 6.678 & 6.698 & 6.713 & 6.724 \\
\hline$L_{1}^{2}$ & 6435 . & 6379 & 6328. & 6289. & 6253. & 0221. & 6192. \\
\hline$L \frac{2}{2}$ & 529.9 & 724.6 & 900.3 & 1048. & 1178. & 1295. & 1402. \\
\hline$p$ & .3323 & .4188 & .4824 & .5235 & .5557 & .5815 & .6028 \\
\hline
\end{tabular}

COMPOSITION BY WEIGHT FRACTION:

$$
\text { Si } 1.0
$$

REFERENCE:
DENSITY VARIATIONS AND REFERENCE

$\mathrm{HCP} \quad 2.42 \mathrm{gm} / \mathrm{cm}^{3}$ RH $\quad 2.33 \mathrm{gm} / \mathrm{cm}^{3}$

material: Silicón Carbide

\begin{tabular}{|c|c|c|c|c|c|c|c|}
\hline TEMPERATURE: & $60 F$ & $300 \mathrm{~F}$ & $1000 \% \mathrm{~F}$ & $1500 \mathrm{~F}$ & $2000 \mathrm{~F}$ & $2500 \mathrm{FF}$ & $3000 \mathrm{~F}$ \\
\hline$D_{1}$ & 1.110 & 1.112 & 1.113 & 1.114 & 1.115 & 1.116 & 1.117 \\
\hline$D_{2}$ & 1.047 & 1.043 & 1.042 & 1.041 & 1.040 & 1.040 & 1.040 \\
\hline$\Sigma_{a_{1}}$ & .0004920 & .0003879 & .0003275 & .0002908 & .0002650 & .0002457 & .0002307 \\
\hline$\Sigma_{a_{2}}$ & .005695 & .004223 & .003425 & .002955 & .002638 & .002405 & .002225 \\
\hline$\Sigma_{s_{1}}$ & .001778 & .001934 & .002035 & .002102 & .002153 & .002193 & .002226 \\
\hline$d$ & 2.334 & 2.324 & 2.316 & 2.310 & 2.305 & 2.302, & 2.299 \\
\hline$d_{1}$ & 2.366 & 2.370 & 2.373 & 2.375 & 2.377 & 2.379 & 2.380 \\
\hline$d_{2}$ & 2.231 & 2.223 & 2.220 & 2.218 & 2.218 & 2.217 & 2.217 \\
\hline$L_{1}^{2}$ & 489.1 & 478.8 & 471.1 & 465.7 & 461.3 & 457.6 & 454.5 \\
\hline$L_{2}^{2}$ & 183.8 & 247.0 & 304.1 & 352.2 & 394.5 & 432.6 & 467.7 \\
\hline$p$ & .7832 & .8329 & .8614 & .8785 & .8904 & .8992 & .9061 \\
\hline
\end{tabular}

COMPOSITION BY WEIGHT FRACTION:

DEISITY VARIATIONS AND REFERENCE

ANPD: E. A. Aitken

$$
\quad 3.21 \mathrm{gm} / \mathrm{cm}^{3}
$$




\begin{tabular}{|c|c|c|c|c|c|c|c|}
\hline IEMPERATURE: & of & Sovor & $1000 \%$ & $1500=p$ & $2000 \%$ & $20000 \mathrm{r}$ & $30000 r$ \\
\hline \multirow[t]{2}{*}{$D_{1}$} & 1.417 & 1.421 & 1.423 & 1,425 & 1.426 & 1.427 & J.. 427 \\
\hline & & & \multicolumn{5}{|c|}{$i$} \\
\hline$D_{2}$ & 1.235 & 1.287 & 1.313 & 1. 327 & 1.336 & 1. 342 & 1. 346 \\
\hline$\Sigma_{a_{1}}$ & .0002793 & .0002223 & .0001882 & .0001667 & .0001520 & .0001410 & .0001323 \\
\hline 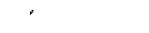 & & & & & $\because$ & & \\
\hline \multirow{2}{*}{$\Sigma_{a_{2}}$} & .003071 & .002277 & .001847 & .001594 & .001422 & .001296 & .001199 \\
\hline & & & & & & & 1 \\
\hline$\Sigma_{s_{1}}$ & .001241 & .001334 & .001397 & 001441 & .001473 & .001500 & .001521 \\
\hline$d$ & 2.909 & 2.923 & 2,932 & 2.938 & 2.941 & 2.944 & 2.945 \\
\hline$\dot{d}_{1}$ & 3.021 & 3.029 & 3,034 & 3.036 & 3.039 & 3.041 & 3.042 \\
\hline$d_{2}$ & 2.632 . & 2.743 & 2.799 & 2,829 & 2.848 & 2.860 & 2.868 \\
\hline$L_{1}^{2}$ & 932.2 & 912,9 & 897.6 & 886.1 & 877.1 & 869.5 & 863.1 \\
\hline$L \frac{2}{2}$ & 402.1 & 565.1 & 711.1 & 832.9 & 939.3 & 1035. & 1122. \\
\hline$p$ & .8163 & .8572 & .8813 & .8963 & .9065 & .9141 & .9200 \\
\hline
\end{tabular}

COMPOSITION BY WEIGHT FRACTION:

$\mathrm{Si} \quad .4756$

REFERENCE:

O . 5244
Material: Sodium
DENSITY VARIATIONS AND REFEREMCE.

ANFD: E. A. Aitken

$2.65 \mathrm{gm} / \mathrm{cm}^{3}$

\begin{tabular}{|c|c|c|c|c|c|c|c|}
\hline TEMPERATURE: & $\cos F$ & $800 \mathrm{~F}$ & $1000 \mathrm{~F}$ & $1500^{\circ} \mathrm{F}$ & $2000 \mathrm{~F}$ & $2500 \mathrm{~F}$ & $3000 \mathrm{~F}$ \\
\hline$D_{1}$ & 4.397 & 4.400 & 4.402 & 4.405 & 4.407 & 4.409 & 4.410 \\
\hline$D_{2}$ & 3.517 & 3.662 & 3.754 & 3.816 & 3.860 & 3.892 & 3.919 \\
\hline$\Sigma_{a_{1}}$ & .0004658 & .0004334 & .0004065 & .0003871 & .0003713 & .0003580 & .0003465 \\
\hline$\Sigma_{a_{2}}$ & .01140 & .008450 & .006852 & .005913 & .005277 & .004812 & .004450 \\
\hline$\Sigma_{s_{1}}$ & .00008438 & .0001189 & .0001479 & .0001693 & .0001869 & .0002019 & .0002150 \\
\hline$d$ & 9.358 & 9.356 & 9.355 & 9.354 & 9.353 & 9.353 & 9.352 \\
\hline$d_{1}$ & 9.372 & 9.378 & 9.384 & 9.389 & 9.393 & 9.397 & 9.400 \\
\hline$d_{2}$ & 7.498 & 7.805 & 8.002 & 8.133 & 8.228 & 8.297 & 8.354 \\
\hline$L_{1}^{2}$ & 7992. & 7966. & 7941. & 7916. & 7894. & 7874. & 7854. \\
\hline$L_{1}^{2}$ & 308.6 & 433.3 & 547.9 & 645.3 & 731.5 & 809.0 & 880.7 \\
\hline$p$ & .1534 & .2153 & .2668 & .3043 & .3348 & .3606 & .3829 \\
\hline
\end{tabular}

COMPOSITION BY WEIGHT FRACTION:

$\mathrm{Na} 1.00$
ASSUMEO DENSITY: $\quad .971 \mathrm{pm} / \mathrm{cm}$ 


\begin{tabular}{|c|c|c|c|c|c|c|c|}
\hline TEMPERATURE: & $\omega 0 \mathrm{~F}$ & $500 \% \mathrm{~F}$ & $1000 \mathrm{FF}$ & $1500 \mathrm{~F}$ & $2000 \mathrm{r}$ & $2500 \mathrm{~F}$ & $3000 \mathrm{~F}$ \\
\hline$D_{1}$ & 1.035 & 1.035 & 1. 0,35 & 1.035 & 1.035 & 1.035 & 1.035 \\
\hline$D_{2}$ & .3167 & .3237 & .3360 & .3424 & .3468 & .3497 & .3519 \\
\hline$\Sigma_{a_{1}}$ & .001307 & .001292 & .001276 & .001262 & .0012 .49 & .001237 & .00 .1226 \\
\hline$\Sigma_{a_{2}}$ & .2103 & .1559 & .1264 &, 1091 & .09741 & .08880 & .0821 .3 \\
\hline$\Sigma_{s_{1}}$ & .00002145 & .00003733 & .00005281 & .00006736 & .00008040 & .00009237 & .0001035 \\
\hline$d$ & 2,205 & 2.205 & 2.205 & 2.205 & 2.205 & $2,2,05$ & 2,205 \\
\hline$d_{1}$ & 2,206 & 2.206 & 2,206 & 2,206 & 2.206 & 2.207 & 2.207 \\
\hline$d_{2}$ & .6750 & .6899 & .7163 & .7298 &, 7392 &, 7454 & .75001 \\
\hline$L_{1}^{2}$ & 778,8 & 778.7 & 778.7 & 778.7 & 778.6 & 778.6 & 778.6 \\
\hline$L_{2}^{2}$ & 1.506 & 2.076 & 2.658 & 3,137 & 3.560 & 3.938 & 4. 284 \\
\hline$p$ & .01615 & .02810 & .03974 & .05067 & .06048 & .06947 & .07784 \\
\hline
\end{tabular}

COMPOSITION BY WEIGHT FRACTION:

REFERENCE:

$$
\begin{array}{llll}
\mathrm{Fe} & .75 & \mathrm{Ni} & .076 \\
\mathrm{Cr} & .174 & &
\end{array}
$$

ANPD: E. A. Aitken
DeNSITY VARIATIONS AND REFEREMCE. ANPD: E. A. Aitken

$7.87 \mathrm{gm} / \mathrm{cm}^{3}$ MH $\quad 7.93 \mathrm{gm} / \mathrm{cm}^{3}$

material: 304 Stainless Steel

\begin{tabular}{|c|c|c|c|c|c|c|c|}
\hline TEMPERATURE: & $\cos F$ & soor $F$ & $1000 \mathrm{~F}$ & $15000 \mathrm{~F}$ & $2000 \mathrm{~F}$ & $25000 \mathrm{~F}$ & $3000 \mathrm{~F}$ \\
\hline$D_{1}$ & 1.030 & 1.030 & 1.030 & 1.031 & 1.031 & 1.031 & 1.031 \\
\hline$D_{2}$ & .3149 & .3224 & .3346 & .3409 & .3454 & .3483 & .3505 \\
\hline$\Sigma_{a_{1}}$ & .001334 & .001318 & .001303 & .001288 & .001275 & .001263 & .001252 \\
\hline$\Sigma_{a_{2}}$ & .2138 & .1585 & .1285 & .1109 & .09903 & .09027 & .08350 \\
\hline$\Sigma_{s_{1}}$ & .00002169 & .00003778 & .00005348 & .00006824 & .00008148 & .00009363 & .0001050 \\
\hline d & 2. 196 & 2.196 & 2.196 & 2. 196 & 2. 196 & 2.196 & 2.196 \\
\hline$d_{1}$ & 2.196 & 2.196 & 2.196 & 2.197 & 2.197 & 2.197 & 2.197 \\
\hline$d_{2}$ & .6713 & .6872 & .7132 & .7267 & .7362 & .7425 & .7472 \\
\hline$L_{1}^{2}$ & 760.0 & 759.9 & 759.9 & 759.9 & 759.8 & 759.8 & 759.7 \\
\hline$L \frac{2}{2}$ & 1.473 & 2.034 & 2.603 & 3.073 & 3.488 & 3.859 & 4.198 \\
\hline$p$ & .01600 & .02786 & .03944 & .05031 & .06006 & .06902 & .07735 \\
\hline
\end{tabular}

ASSUMED DENSITY: $\quad 7.94 \mathrm{gm} / \mathrm{Cm}^{\circ}$

COMPOSITION BY WEIGHT FRACTION:

REFERENCE:

$$
\begin{array}{llll}
C & .0008 \quad \mathrm{Ni} & .095
\end{array}
$$

$\begin{array}{llll}\mathrm{C} & .0008 & \mathrm{Ni} & .095 \\ \mathrm{Cr} & .19 & \mathrm{Fe} & .7142\end{array}$

ANPD: E. A. Aitken
DENSITY VARIATIONS AND REFERENCE:

ANPD: E. A. Aitken

$7.94 \mathrm{gm} / \mathrm{cm}^{3}$ 


\begin{tabular}{|c|c|c|c|c|c|c|c|}
\hline TEMPERATURE: & $\Delta F$ & soor & $1000 \% F$ & $1500 \% F$ & $2000 \mathrm{~F}$ & $2500 \mathrm{~F}$ & $3000 \mathrm{~F}$ \\
\hline$D_{1}$ & 1.033 & 1.033 & 1.033 & 1.033 & 1.033 & 1.033 & 1.033 \\
\hline$D_{2}$ & .3050 & .3143 & .3257 & .3319 & .3364 & .3395 & .3418 \\
\hline$\Sigma_{a_{1}}$ & .001370 & .001355 & .001340 & .001326 & .001314 & .001302 & .001292 \\
\hline$\Sigma_{a_{2}}$ & .2312 & .1715 & .1390 & .1200 & .1071 & .09764 & $.0903 i$ \\
\hline$\Sigma_{s_{1}}$ & .00002011 & .00003532 & .00005023 & .00006438 & .00007709 & .00008878 & .00009970 \\
\hline$d$ & 2.201 & 2.201 & 2.201 & 2.201 & 2.201 & 2.201 & 2.201 \\
\hline$d_{2}$ & 2.201 & 2.201 & 2.202 & 2.202 & 2.202 & 2.202 & 2. 202 \\
\hline$d_{2}$ & .6501 & .6699 & .6943 & .7075 & .7170 & .7236 & .7286 \\
\hline$L_{1}^{2}$ & 742.8 & 742.8 & 742.8 & 742.8 & 742.7 & 742.7 & 742.7 \\
\hline$L \frac{2}{2}$ & 1.319 & 1. 833 & 2.343 & 2.766 & 3.141 & 3.477 & 3.785 \\
\hline$p$. & .01446 & .02540 & .03613 & .04629 & .05542 & .06382 & .07167 \\
\hline
\end{tabular}

COMPOSITION EY WEIGHT FRACTION:

$\begin{array}{lllll} & \mathrm{Fe} & .559 & \mathrm{Ni} & .191 \\ \text { REFERENCE: } & \mathrm{Cr} & .25 & & \end{array}$

ANPD: E. A. Aitken
DENSITY VARIATIONS AND REFERENCE:

ANPD: E. A. Aitken

$7.98 \mathrm{gm} / \mathrm{cm}^{3}$

MH $\quad 7.98 \mathrm{gm} / \mathrm{cm}^{3}$

$\mathrm{RH} \quad 7.92 \mathrm{gm} / \mathrm{cm}^{3}$

material: 316 Stainless Steel

ASSUMEO DENSITY: $\quad 7.98 \mathrm{gm/L=}$

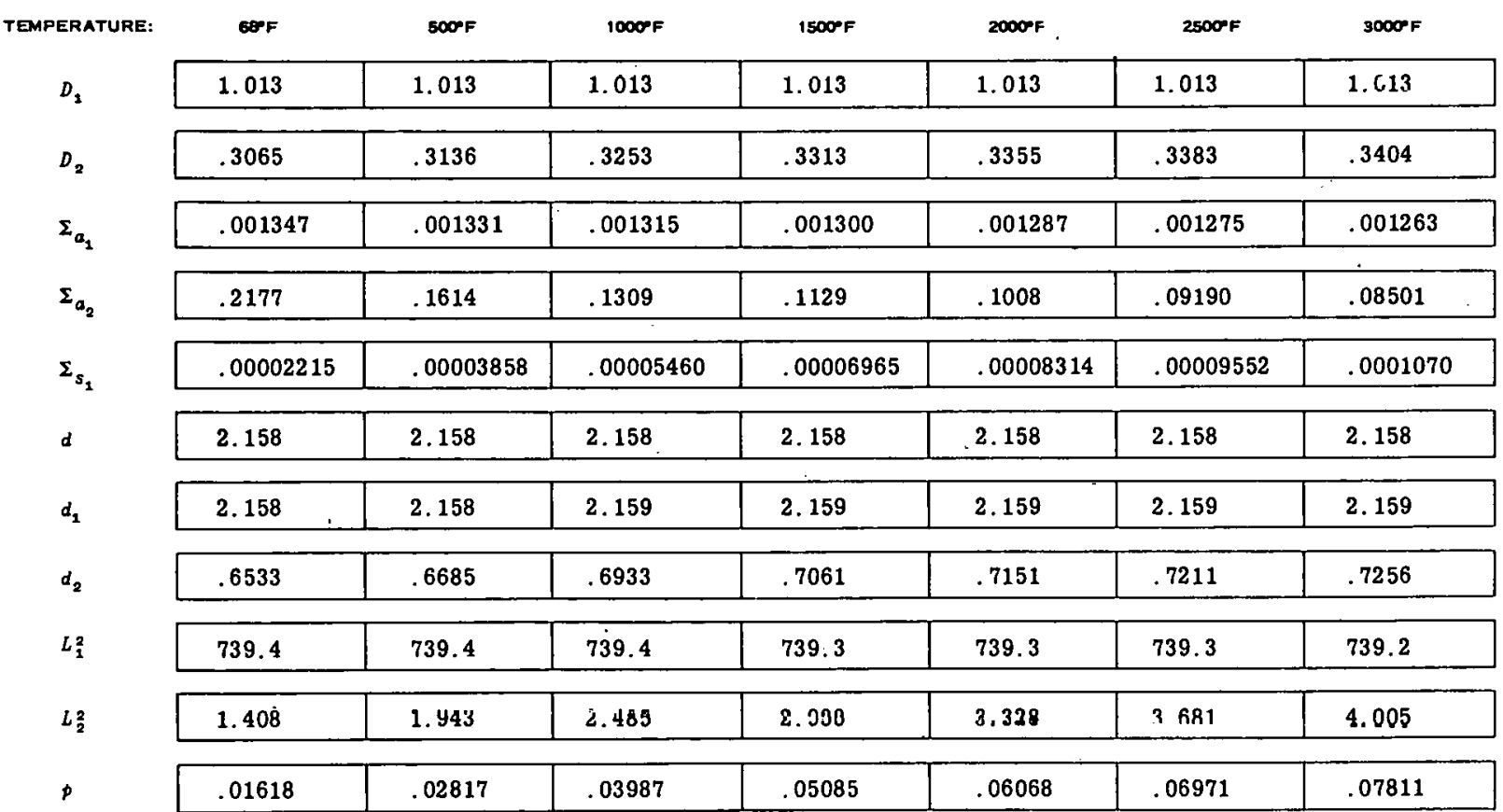

COMPOSITION GY WEIGHT FRACTION:

REFERENCE:

$$
\begin{array}{llll}
\mathrm{Fe} & .71 & \mathrm{Ni} & .12 \\
\mathrm{Cr} & .17 & &
\end{array}
$$

ANPD: E. A. Aitken
DENSITY VARIATIONS AND REFERENCE:

ANPD: E. A. Aitken

$$
\begin{aligned}
& 8.03 \mathrm{gm} / \mathrm{cm}^{3} \\
& 7.98 \mathrm{gm} / \mathrm{cm}^{3}
\end{aligned}
$$




\begin{tabular}{|c|c|c|c|c|c|c|c|}
\hline EMPERATURE: & $600 \mathrm{~F}$ & $500 \mathrm{~F}$ & $1000 \mathrm{~F}$ & $1500 \mathrm{~F}$ & $2000 \mathrm{~F}$ & $2500^{\circ} \mathrm{F}$ & $3000 \% \mathrm{~F}$ \\
\hline$D_{1}$ & 1.056 & 1,056 & 1.056 & 1.056 & 1.056 & 1,056 & 1.056 \\
\hline$D_{2}$ & .3207 & .3283 & .3404 & .3467 & .3512 & .3541 & .3563 \\
\hline$\Sigma_{a_{1}}$ & .001380 & .001371 & .001363 & .001355 & .001348 & .001341 & .001335 \\
\hline$\Sigma_{a_{2}}$ &, 2092 & .1551 & $\begin{array}{r}1.258 \\
\end{array}$ & .1086 & .09690 & .08833 & .08170 \\
\hline$\Sigma_{s_{1}}$ & .00001201 & .00002092 & .00002961 & $: 00003778$ & .00004510 & .00005182 & .00005807 \\
\hline$d$ & 2.251 & 2.251 & 2.250 & 2,250 & 2.250 & 2.250 & 2.250 \\
\hline$d_{1}$ & 2.251 & 2.251 & 2.251 & 2,251 & 2,251 & 2.251 & 2.251 \\
\hline$d_{2}$ & .6836 & 6999 & .7256 & .7390 &, 7485 & .7548 & .7596 \\
\hline$L_{1}^{2}$ & 758.4 & 758.4 & 758.3 & 758,3 & 758.3 & 758.3 & 758.3 \\
\hline$L \frac{2}{2}$ & 1.533 & 2.117 & 2.707 & 3.194 & 3.624 & 4.009 & 4.361 \\
\hline$p$ & .008626 & .01502 & .02127 & .02713 & .03238 & .03720 & .04169 \\
\hline
\end{tabular}

COMPOSITION GY WEIGHT FRACTION:

$$
\begin{array}{llllll}
\mathrm{Cr} & .17 & \mathrm{Mo} & .025 & \mathrm{Fe} & .684
\end{array}
$$

REFERENCE:

ANPD: E. A. Aitken

DENSITY VARIATIONS AND REFERENCE:

ANPD: E. A. Aitken

$7.70 \mathrm{gm} / \mathrm{cm}^{3}$

MH $\quad 7.75 \mathrm{gm} / \mathrm{cm}^{3}$

RH $\quad .7 .75 \mathrm{gm} / \mathrm{cm}^{3}$ material: 430 Stainless Steel

ASSUMED DENSITY: $\quad 7.70 \mathrm{gm} / \mathrm{cm}$

\begin{tabular}{|c|c|c|c|c|c|c|c|}
\hline TEMPERATURE: & $60 F$ & SOOFF & $1000 \mathrm{~F}$ & $1500^{\circ} \mathrm{F}$ & $20000 \mathrm{~F}$ & $20500 \mathrm{~F}$ & $3000 \mathrm{~F}$ \\
\hline$D_{1}$ & 1.081 & 1.081 & 1.081 & 1.081 & 1.081 & 1.082 & 1.082 \\
\hline$D_{2}$ & .3384 & .3446 & .3584 & .3653 & .3700 & .3730 & .3752 \\
\hline$\Sigma_{a_{1}}$ & .001242 & .001227 & .001212 & .001198 & .001185 & .001174 & .001163 \\
\hline$\Sigma_{a_{2}}$ & .1930 & .1431 & .1161 & .1002 & .08941 & .08151 & .07539 \\
\hline$\Sigma_{s}$ & .00002144 & .00003712 & .00005236 & .00006662 & .00007940 & .00009112 & .0001020 \\
\hline d & 2.304 & 2.304 & 2.304 & 2.304 & 2.304 & 2.304 & 2.304 \\
\hline$d_{2}$ & .7213 & .7346 & .7640 & .7785 & .7887 & .7952 & .7998 \\
\hline$L 2$ & 855.4 & 855.4 & 855.4 & 855.3 & 855.3 & 855.2 . & 855.2 \\
\hline$L \frac{2}{2}$ & 1.753 & 2.408 & 3.088 & 3.646 & 4.138 & 4.577 & 4.977 \\
\hline$p$ & .01696 & .02937 & .04142 & .05269 & .06279 & .07205 & .08065 \\
\hline
\end{tabular}

COMPOSITION EY WEIGHT FRACTION:

REFERENCE:

$$
\text { C } \quad .0012 \quad \mathrm{Fe} \quad .8388
$$$$
\begin{array}{ll}
\mathrm{C} & .0012 \\
\mathrm{Cr} & .16
\end{array}
$$

ANPD: E. A. Aitken
DENSITY VARIATIONS AND REFERENCE:

ANPD: E. A. Aitken

$7.75 \mathrm{gm} / \mathrm{cm}^{3}$

MH $\quad 7.70 \mathrm{gm} / \mathrm{cm}^{3}$
RH $\quad 7.70 \mathrm{gm} / \mathrm{cm}^{3}$ 


\begin{tabular}{|c|c|c|c|c|c|c|c|}
\hline TEMPERATURE: & CorF & $500 \mathrm{~F}$ & $1000^{\circ} \mathrm{F}$ & $1500^{\circ} \mathrm{F}$ & $20000 \mathrm{~F}$ & $25000 \mathrm{~F}$ & $3000 \mathrm{~F}$ \\
\hline$D_{1}$ & 1.132 & 1.133 & 1.133 & 1.133 & 1.133 & 1.133 & 1.133 \\
\hline$D_{2}$ & .3643 & .3746 & .3905 & .3989 & .4047 & .4086 & 4115 \\
\hline$\Sigma_{a_{1}}$ & .001262 & .001248 & .001235 & .001223 & .001212 & .001201 & .001192 \\
\hline$\Sigma_{a_{2}}$ & .1994 & 1478 & .1199 & .1035 & .09234 & .08418 & .07786 \\
\hline$\Sigma_{s_{1}}$ & .00001790 & .00003141 & .00004464 & .00005724 & .00006861 & .00007908 & .00008886 \\
\hline$d$ & 2.414 & 2.414 & 2.414 & 2.414 & 2.413 & 2.413 & 2.413 \\
\hline$d_{1}$ & 2.414 & 2.414 & 2.414 & 2.414 & 2.415 & 2.415 & 2.415 \\
\hline$d_{2}$ & .7765 & .7984 & .8324 & .8502 & .8627 & .8710 & .8772 \\
\hline$L_{1}^{2}$ & 885.0 & 885.0 & 884.9 & 884,9 & 884.9 & 884.8 & 884.8 \\
\hline$L \frac{2}{2}$ & 1.827 & 2.534 & 3.258 & 3.855 & 4.383 & 4.854 & 5.285 \\
\hline$p$ & .01399 & .02454 & .03488 & .04472 & .05359 & .06177 & .06939 \\
\hline
\end{tabular}

COMPOSITION BY WEIGHT FRACTION:
$\begin{array}{llllllll}\mathrm{C} & .002 & \mathrm{Fe} & .6975 & \mathrm{Nb} & .005 & \mathrm{~N} & .0025\end{array}$

$\begin{array}{llllllll}\mathrm{Cr} & .27 & \mathrm{Mn} & .008 & \mathrm{Ni} & .005 & \mathrm{Si} & .010\end{array}$
DENSITY VAAIATIONS AND REFERENCE:

ANPD: F. D. Kingsbury $7.60 \mathrm{gm} / \mathrm{cm}^{3}$

ANPD: F. D. Kingsbury

MATERIAL: THERMOFLEX

ASSUMEO DENSITY: $\quad .224 \mathrm{gm} / \mathrm{\sigma}=0$

$1500 \%$

$20000 \%$

2500\%

$3000 \mathrm{~F}$

\begin{tabular}{|c|c|c|c|c|c|c|c|}
\hline EMPERATURE: & $\cos F$ & somp & $1000 \mathrm{~F}$ & $1500 \% \mathrm{~F}$ & $2000,0 \mathrm{~F}$ & $2500 \mathrm{~F}$ & $3000 \mathrm{~F}$ \\
\hline$D_{1}$ & 17.38 & 17.41 & 17.42 & 17.43 & 17.44 & 17.44 & 17.45 \\
\hline$D_{2}$ & 15.01 & 15.77 & 16.18 & 16.40 & 16.55 & 16.64 & 16.71 \\
\hline$\Sigma_{a_{1}}$ & .00006664 & .00005663 & .00004979 & .00004528 & .00004202 & .00003948 & .00003741 \\
\hline$\Sigma_{a_{2}}$ & .0009646 & .0007152 & .0005798 & .0005005 & .0004467 & .0004072 & .0003768 \\
\hline$\Sigma_{s_{1}}$ & .00006480 & .00007659 & .00008495 & .00009078 & .00009510 & .00009858 & .0001015 \\
\hline$d$ & 36.74 & 36.77 & 36.80 & 36.82 & 36.84 & 36.85 & 36.86 \\
\hline$d_{1}$ & 37.06 & 37.11 & 37.14 & 37.15 & 37.17 & 37.18 & 37.19 \\
\hline$d_{2}$ & 31.99 & 33.61 & 34.48 & 34.96 & 35.27 & 35.48 & 35.63 \\
\hline$L_{1}^{2}$ & 132300 & 130700 & 129300 & 128100 . & 127200 . & 126400 . & 125600 \\
\hline$L \frac{2}{2}$ & $15500 \hat{.}$ & 22050 . & 37000 & $3277 n$. & $37 n 4 n$. & 40880. & 44360 . \\
\hline$p$ & .4930 & .5749 & .6306 & .6672 & .6936 & .7141 & .7306 \\
\hline
\end{tabular}

COMPOSITION BY WEIGHT FRACTION:

REFERENCE:
DENSITY VARIATIONS AND REFERENCE: Arbitrary $.224 \mathrm{gm} / \mathrm{cm}^{3}$

ANPD: T. McClay 


\begin{tabular}{|c|c|c|c|c|c|c|c|}
\hline TEMPERATURE: & 6of & $500 \%$ & $1000 \mathrm{~F}$ & $1500 \mathrm{~F} F$ & $2000 \mathrm{~F}$ & $2500 \% \mathrm{~F}$ & $3000 \% F$ \\
\hline$D_{1}$ & 1. 532 & 1.532 & 1.532 & 1.532 & 1.532 & 1.532 & 1. 532 \\
\hline$D_{2}$ & .5843 & .6390 & .6724 & .6892 & .7090 & $.7211^{\circ}$ & .7310 \\
\hline$\Sigma_{a_{1}}$ & .002754 & .002754 & .002754 & .002754 & .002754 & .002754 & .002754 \\
\hline & & & 1147 & 1021 & 08845 & & \\
\hline${ }^{2} a_{2}$ & .1902 & .1412 & .1147 & .1021 & .08845 & .08061 & .07451 \\
\hline$\Sigma_{s_{1}}$ & $1.032 \times 10^{-13}$ & $2.043 \times 10^{-13}$ & $3.096 \times 10^{-13}$ & $4.336 \times 10^{-13}$ & $5.520 \times 10^{-13}$ & $6.647 \times 10^{-13}$ & $7.740 \times 10^{-13}$ \\
\hline$d$ & 3,266 & 3.266 & 3.266 & 3.266 & 3.260 & 3.266 & 3.266 \\
\hline$d_{1}$ & 3.266 & 3.266 & 3.266 & 3.266 & 3. 266 & 3.266 & 3.266 \\
\hline$d_{2}$ & $\cdot 1.245$ & 1.362 & 1.433 & 1.469 & 1.511 & 1.537 & 1.558 \\
\hline$L_{1}^{2}$ & 556.2 & 556,2 & 556.2 & 556.2 & 556.2 & 556.2 & 536.2 \\
\hline$L_{2}^{2}$ & 3.073 & 4.524 & 5.863 & 6.748 & 8.017 & 8.946 & 9.811 \\
\hline$p$ & $3.746 \times 10^{-11}$ & $7.417 \times 10^{-11}$ & $1.124 \times 10^{-10}$ & $1.574 \times 10^{-10}$ & $2.004 \times 10^{-10}$ & $2.413 \times 10^{-10}$ & $2.810 \times 10^{-10}$ \\
\hline
\end{tabular}

COMPOSITION EY WEIGHT FRACTION:

Th 1.00
DENSITY VARIATIONS AND REFERENCE: HCP

$11.85 \mathrm{gm} / \mathrm{cm}^{3}$

REFERENCE:

material: Titanium

ASSUMEO DENSITY: $\quad 4,507 \mathrm{gm} / \mathrm{cm}^{\circ}$

\begin{tabular}{|c|c|c|c|c|c|c|c|}
\hline TEMPERATURE: & ErF & $500 \mathrm{~F}$ & $1000 \mathrm{~F}$ & $1500 \mathrm{~F}$ & $20000 \mathrm{~F}$ & $2500{ }^{\circ} \mathrm{F}$ & $9000^{\circ} \mathrm{F}$ \\
\hline$D_{1}$ & 1. 063 & 1.063 & 1.063 & 1.063 & 1.063 & 1.063 & 1.063 \\
\hline$D_{2}$ & .5963 & .7175 & .8017 & .8588 & .9012 & .9345 & .9615 \\
\hline$\Sigma_{a}$ & .001720 & .001718 & .001716 & .001714 & .001712 & .001711 & .001709 \\
\hline$\Sigma_{a_{2}}$ & .2816 & .2088 & .1693 & .1461 & .1304 & .1189 & .1100 \\
\hline$\Sigma_{s}$ & $1.842 \times 10^{-6}$ & $3.558 \times 10^{-6}$ & $5.328 \times 10^{-6}$ & $7.312 \times 10^{-6}$ & $9.182 \times 10^{-6}$ & .00001095 & .00001266 \\
\hline$d$ & 2.266 & 2.266 & 2.266 & 2.266 & 2.266 & 2.266 & 2.266 \\
\hline$d_{1}$ & 2.266 & 2.266 & 2.266 & 2.266 & 2.266 & 2.266 & 2.266 \\
\hline$d_{2}$ & 1.271 & 1. 529 & 1.709 & 1.830 & 1.921 & 1.992 & 2.049 \\
\hline$L_{1}^{2}$ & 617.7 & 617.7 & 617.7 & 617.7 & 617.7 & 617.7 & 617.7 \\
\hline$L \frac{2}{2}$ & 2.118 & 3.436 & 4.737 & 5.878 & 6.912 & 7.861 & 8.743 \\
\hline$p$ & .001070 & .002067 & .003095 & .004248 & .005334 & .006363 & .007355 \\
\hline
\end{tabular}

COMPOSITION BY WEIGHT FRACTION:

Ti 1.00
DENSITY VARIATIONS AND REFERENCE

RMH
$4.507 \mathrm{gm} / \mathrm{cm}^{3}$ 


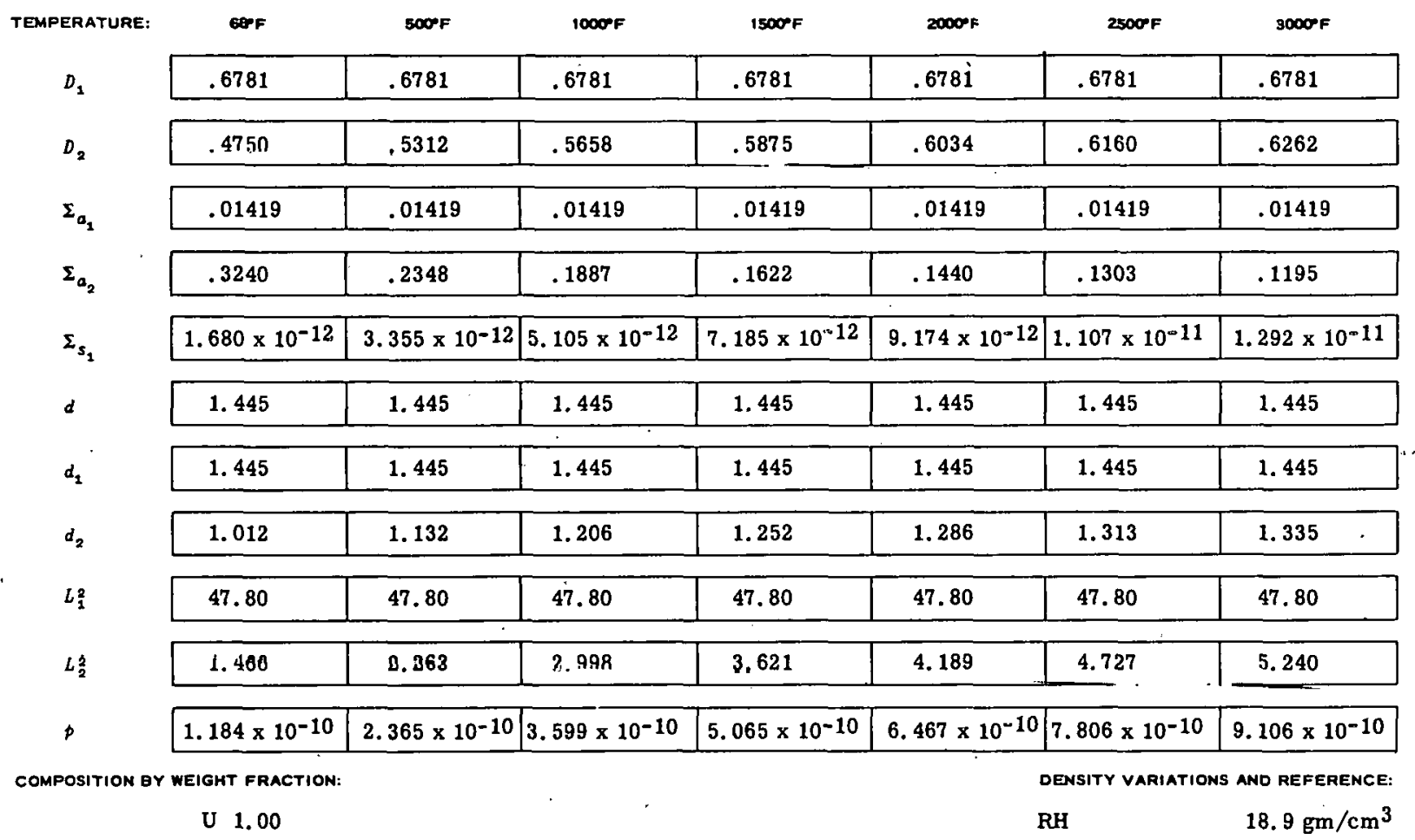

REFERENCE:

material: Tuballoy

\begin{tabular}{|c|c|c|c|c|c|c|c|}
\hline TEMPERATURE: & 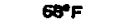 & $500 \mathrm{~F}$ & $1000 \mathrm{~F}$ & $1500 \mathrm{~F}$ & $20000^{\circ}$ & $2500{ }^{\circ} \mathrm{F}$ & $3000^{\circ} \mathrm{F}$ \\
\hline$\nu \Sigma_{f_{2}}$ & .004409 & .004409 & .004409 & .004409 & .004409 & .004409 & .004409 \\
\hline$\nu \Sigma_{f_{2}}$ & .4289 & .3063 & .2437 & .2081 & .1837 & .1654 & .1508 \\
\hline$k_{1}$ & .3108 & .3108 & .3108 & .3108 & .3108 & .3108 & .3108 \\
\hline$k_{2}$ & 1.324 & 1.305 & 1.291 & 1.283 & 1.276 & 1.269 & 1.262 \\
\hline
\end{tabular}




\begin{tabular}{|c|c|c|c|c|c|c|c|}
\hline TEMPERATURE: & $600 \mathrm{~F}$ & $500 \% \mathrm{~F}$ & $1000 \mathrm{~F}$ & $1500 \mathrm{~F}$ & $2000 \mathrm{FF}$ & $2500 \mathrm{~F}$ & $3000 \mathrm{~F}$ \\
\hline$D_{1}$ & .6534 & .6534 & .6534 & .6534 & .6534 & .6534 & .6534 \\
\hline$D_{2}$ & .2558 & .3117 & .3551 & .3876 & .4137 & .4357 & .4546 \\
\hline$\Sigma_{a}$ & .001 .014 & .001014 & .001014 & .0020 .14 & .005014 & .001014 & .00101 .4 \\
\hline$\Sigma_{a_{2}}$ & i. 065 & .7947 & .6457 & .5577 & .4981 & .4542 & .4204 \\
\hline$\Sigma_{s_{1}}$ & $2.792 \times j 0^{\circ 8}$ & $5.642 \times 10^{-8}$ & $8.634 \times 10^{-8}$ & $1.228 \times 10^{-7}$ & 1. $577 \times 10^{-7}$ & $1.911 \times 10^{-7}$ & $2.237 \times 10^{-7}$ \\
\hline$d$ & 1. 393 & 1. 393 & 1.393 & 1.393 & 1.393 & i. 393 & i. 393 \\
\hline$d_{2}$ & 1.393 & 1.393 & 1.393 & 1.393 & 1.393 & 1.0393 & 3.393 \\
\hline$d_{2}$ & .5453 & .6644 & .7570 & .8262 & .8819 & .9287 & .9690 \\
\hline$L_{1}^{2}$ & 644.5 & 644.5 & 644.5 & 644.5 & 644.5 & 644.5 & 644.5 \\
\hline$L_{2}^{2}$ & .2401 & .3922 & .5500 & .6950 & .8306 & .9591 & i. 081 \\
\hline$p$ & .00002754 & .00005565 & .00008516 & .0001211 & $.0001555^{\circ}$ & .0001885 & .0002206 \\
\hline
\end{tabular}

COMPOSITION BY WEIGHT FRACTION:

W 1.00

REFERENCE: material: Tungsten Carbide
OENSITY VARIATIONS AND REFERENCE: HCP RH

$19.3 \mathrm{gm} / \mathrm{cm}^{3}$ 19. $3 \mathrm{gm} / \mathrm{cm}^{3}$

\begin{tabular}{|c|c|c|c|c|c|c|c|}
\hline TEMPERATURE: & $60 \%$ & $500 \mathrm{~F}$ & $1000 \mathrm{~F}$ & $1500^{\circ} \mathrm{F}$ & $2000 \mathrm{~F}$ & $25000 \mathrm{~F}$ & $3000 \% \mathrm{~F}$ \\
\hline$D_{1}$ & .5693 & .5693 & .5693 & .5693 & .5693 & .5693 & .5693 \\
\hline$D_{2}$ & .2771 & .3243 & .3586 & .3829 & .4018 & .4172 & .4301 \\
\hline$\Sigma_{a_{1}}$ & .003546 & .003543 & .003541 & .003538 & .003536 & .003533 & .003531 \\
\hline$\Sigma_{a_{2}}$ & .8101 & .6042 & .4909 & .4241 & .3787 & .3454 & .3196 \\
\hline$\Sigma_{s_{1}}$ & $2.636 \times 10^{-6}$ & $5.024 \times 10^{-6}$ & $7.473 \times 10^{-6}$ & .00001015 & .00001267 & .00001505 & .00001733 \\
\hline$d$ & 1.213 & 1.213 & 1.213 & 1.213 & 1.213 & 1.213 & 1.213 \\
\hline$d_{1}$ & 1.213 & 1.213 & 1.213 & 1.213 & 1.213 & 1.213 & 1.213 \\
\hline$d_{2}$ & .5907 & .6913 & .7644 & .8163 & .8564 & .8892 & .9168 \\
\hline$L_{1}^{2}$ & 160.4 & 160.4 & 160.4 & 160.4 & 160.4 & 160.4 & 160.4 \\
\hline$L_{2}^{2}$ & .3421 & .5368 & .7305 & . 9030 & 1.061 & 1.208 & 1.346 \\
\hline$p$ & .0007428 & .001416 & .002106 & .002861 & .003572 & .004242 & .004885 \\
\hline
\end{tabular}

COMPOSITION BY WEIGHT FRACTION:

DENSITY VARIATIONS AND REFERENCE: 


\begin{tabular}{|c|c|c|c|c|c|c|c|}
\hline TEMPERATURE: & $60^{\circ} \mathrm{F}$ & $5000^{\circ} \mathrm{F}$ & $1000^{\circ} \mathrm{F}$ & $1500^{\circ} \mathrm{F}$ & $2000 \% \mathrm{~F}$ & $2500^{\circ} \mathrm{F}$ & $300 \mathrm{PF}$ \\
\hline$D_{1}$ & .5790 & .5790 & .5790 & .5790 & .5790 & .5790 & .5790 \\
\hline$D_{2}$ & .01324 & .01786 & .02184 & .02497 & .02750 & .02956 & .03116 \\
\hline$\Sigma_{a_{1}}$ & .09910 & .09910 & .09910 & .09910 & .09910 & .09910 & .09910 \\
\hline$\Sigma_{a_{2}}$ & 24.69 & $18.19^{\circ}$ & 14.79 & 12.87 & 11.64 & 10.80 & 10.22 \\
\hline$\Sigma_{s_{2}}$ & $1.889 \times 10^{-18}$ & $3.835 \times 10^{-18}$ & $5.880 \times 10-18$ & $8.392 \times 10^{-18}$ & $1.080 \times 10^{-17}$ & $1.311 \times 10^{-17}$ & $1.537 \times 10^{-17}$ \\
\hline$d$ & 1.234 & 1.234 & 1.234 & 1.234 & 1.234 & 1.234 & 1.234 \\
\hline$d_{1}$ & 1.234 & 1.234 & 1.234 & 1.234 & 1.234 & 1.234 & 1.234 \\
\hline$d_{2}$ & .02823 & .03807 & .04655 & .05321 & .05862 & .06300 & .06642 \\
\hline$L_{1}^{2}$ & 5.843 & 5.843 & 5.843 & 5.843 & 5.843 & 5.843 & 5.843 \\
\hline$L_{2}^{2}$ & .0005363 & .0009821 & .001477 & .001939 & .002362 & .002736 & $.003049^{\circ}$ \\
\hline$p$ & $1.907 \times 10^{-17}$ & $3.870 \times 10^{-17}$ & $5.934 \times 10^{-17}$ & $8.468 \times 10^{-17}$ & $1.090 \times 10^{-16}$ & $1.323 \times 10^{-16}$ & $1.551 \times 10^{-16}$ \\
\hline
\end{tabular}

COMPOSITION BY WEIGHT FRACTION:

$\mathrm{U}^{233} 1.00$
DENSITY VARIATIONS AND REFERENCE: HCP $18.50 \mathrm{gm} / \mathrm{cm}^{3}$ (computed)

REFERENCE:

MATERIAL: Uranium 235

ASSUMEO DENSITY: $\quad 18.66 \mathrm{gm} / \mathrm{cm}$ '

\begin{tabular}{|c|c|c|c|c|c|c|c|}
\hline TEMPERATURE: & $60^{\circ} \mathrm{F}$ & $5000 \mathrm{~F}$ & $1000 \mathrm{~F}$ & $1500^{\circ} \mathrm{F}$ & $20000 \mathrm{~F}$ & $2500 \mathrm{~F}$ & $3000 \mathrm{~F}$ \\
\hline$D_{1}$ & 1. 473 & 1.473 & 1.473 & 1.473 & 1.473 & 1.473 & 1.173 \\
\hline$D_{2}$ & .01145 & .01592 & .01981 & .02300 & .02590 & .02865 & .03131 \\
\hline$\Sigma_{a_{1}}$ & .07502 & .07502 & .07502 & .07502 & .07502 & .07502 & .07502 \\
\hline$\Sigma_{a_{2}}$ & 28.71 & 20.53 & 16.42 & 14.09 & 12.47 & 11.23 & 10.24 \\
\hline$\Sigma_{s}$, & $5.282 \times 10^{-18}$ & $1.072 \times 10^{-17}$ & $1.644 \times 10^{-17}$ & $2.346 \times 10^{-17}$ & $3.021 \times 10^{-17}$ & $3.667 \times 10^{-17}$ & $4.297 \times 10^{-17}$ \\
\hline$d$ & 3.139 & 3.139 & 3.139 & 3.139 & 3.139 & 3.139 & 3.139 \\
\hline$d_{1}$ & 3.139 & 3.139 & 3.139 & 3.139 & 3.139 & 3.139 & 3.139 \\
\hline$d_{2}$ & .02440 & .03394 & .04223 & .04903 & .05520 & .06108 & .06673 \\
\hline$L_{1}^{2}$ & 19.63 & 19.63 & 19.63 & 19.63 & 19.63 & 19.63 & 19.63 \\
\hline$L \frac{2}{2}$ & .0003988 & .0007754 & .001207 & .001633 & .002078 & .002552 & .003057 \\
\hline$p$ & $7.041 \times 10^{-17}$ & $1.429 \times 10^{-16}$ & $2.192 \times 10^{-16}$ & $3.128 \times 10^{-16}$ & $4.026 \times 10^{-16}$ & $4.888 \times 10^{-16}$ & $5.728 \times 10^{-16}$ \\
\hline
\end{tabular}

COMPOSITION EY WEIGHT FRACTION:

$$
\mathrm{U}^{235} \quad 1.00
$$

DENSITY VARIATIONS ANO REFERENCE: $\mathrm{RH} \quad 18.66 \mathrm{gm} / \mathrm{cm}^{3}$ (computed)

REFERENCE: 
MATERIAL: Uranium 235

\begin{tabular}{|c|c|c|c|c|c|c|c|}
\hline TEMPERATURE: & $68 \mathrm{~F}$ & $500 \mathrm{~F}$ & $10000 \mathrm{~F}$ & $1500 \mathrm{~F}$ & $2000 \mathrm{~F}^{\circ}$ & $2500^{\circ} \mathrm{F}$ & $3000^{\circ} \mathrm{F}$ \\
\hline$\nu \Sigma_{f_{1}}$ & .1522 & .1522 & .1522 & .1522 & .1522 & .1522 & .1522 \\
\hline$v \Sigma_{f_{2}}$ & 59,15 & 42.23 & 33,60 & 28,70 & 25.34 & 22.80 & 20.80 \\
\hline$k_{1}$ & 2.029 & 2.029 & 2.029 & 2.029 & 2.029 & 2.029 & 2.029 \\
\hline & & & & & & & 7 \\
\hline$k_{2}$ & 2.060 & 2.057 & 2.046 & 2.037 & 2.033 & 2.031 & 2.031 \\
\hline
\end{tabular}

MATERIAL: Uranium 238

ASSUMEO DENSITY: $\quad 18.9 \mathrm{gm} / \mathrm{cm} *$

\begin{tabular}{|c|c|c|c|c|c|c|c|}
\hline TEMPERATURE: & $60 \%$ & $500 \mathrm{~F}$ & $1000 \mathrm{~F}$ & $1500 \% \mathrm{~F}$ & $20000 \mathrm{~F}$ & $2500 \mathrm{~F}$ & $3000 \mathrm{~F}$ \\
\hline$D_{3}$ & .6727 & .6727 & .6727 & .6727 & .6727 & .6727 & .6727 \\
\hline$D_{2}$ & .6745 & .6957 & .7084 & .7159 & .7208 & .7245 & .7272 \\
\hline$\Sigma_{a_{1}}$ & .01364 & .01364 & .01364 & .01364 & .01364 & .01364 & .01364 \\
\hline$\Sigma_{a_{2}}$ & .1166 & .08648 & .07012 & .06052 & .05402 & .04924 & .04555 \\
\hline$\Sigma_{s}$ & $2.590 \times 10^{-12}$ & $5.054 \times 10^{-12}$ & $7.604 \times 10^{-12}$ & $1.049 \times 10^{-11}$ & $1.322 \times 10^{-11}$ & $1.581 \times 10^{-11}$ & $1.831 \times 10^{-11}$ \\
\hline$d$ & 1.434 & 1.434 & 1.434 & 1.434 & 1.434 & 1.434 & 1.434 \\
\hline$d_{1}$ & 1.434 & 1.434 & 1.434 & 1.434 & 1.434 & 1.434 & 1.434 \\
\hline$d_{2}$ & 1.438 & 1,483 & 1.510 & 1.526 & 1.536 & 1.544 & 1.550 \\
\hline$L_{1}^{2}$ & 49.32 & 49.32 & 49.32 & 49.32 & 49.32 & 49.32 & 49.32 \\
\hline$L \frac{2}{2}$ & 5.784 & 8.045 & 10.10 & 11.83 & 13.34 & 14.71 & 15.97 \\
\hline$p$ & $1.899 \times 10^{-10}$ & $3.705 \times 10^{-10}$ & $5.575 \times 10^{-10}$ & $7.689 \times 10^{-10}$ & $9.689 \times 10^{-10}$ & $1.159 \times 10^{-9}$ & $1.342 \times 10^{-9}$ \\
\hline
\end{tabular}

COMPOSITION BY WEIGHT FRACTION:

$$
U^{238} \quad 1.00
$$

REFERENCE:

MATERIAL: Uranium 238

\begin{tabular}{|c|c|c|c|c|c|c|c|}
\hline TEMPERATURE: & $\infty F$ & $500 \mathrm{~F}$ & $1000 \%$ & $15000 \mathrm{~F}$ & $2000{ }^{\circ} \mathrm{F}$ & $2500^{\circ} \mathrm{F}$ & $3000 \mathrm{~F}$ \\
\hline$\nu \Sigma_{f_{1}}$ & .002695 & .002695 & .002695 & .002695 & .002695 & .002695 & .002695 \\
\hline$\nu \Sigma_{f_{2}}$ & 0 & 0 & 0 & 0 & 0 & 0 & 0 \\
\hline$k_{1}$ & .1976 & .1976 &, 1976 & .1976 & .1976 & .1976 & .1976 \\
\hline$k$ & 0 & 0 & 0 & 0 & 0 & 0 & 0 \\
\hline
\end{tabular}


MATERIAL: Water

ASSUMEO DENSITY: $\quad .998 \mathrm{gm} / \mathrm{cm}^{\circ}$

\begin{tabular}{|c|c|c|c|c|c|c|c|}
\hline TEMPERATURE: & $\cos F$ & $500 \mathrm{~F}$ & $1000 \mathrm{FF}$ & $1500 \mathrm{~F}$ & $2000 \mathrm{~F}$ & $2500 \%=$ & $3000 \mathrm{~F}$ \\
\hline$D_{2}$ & 1.423 & 1.445 & 1.460 & 1.471 & 1.479 & 1.486 & 1.492 \\
\hline$D_{2}$ & .1469 & .1913 & .2308 & .2626 & .2895 & .3127 & .3331 \\
\hline$\Sigma_{a_{1}}$ & .001188 & .0009054 & .0007523 & .0006598 & .0005967 & .0005503 & .0005140 \\
\hline$\cdot \boldsymbol{\Sigma}_{a_{2}}$ & .01954 & .01449 & .01175 & .01014 & .009049 & .008248 & .007628 \\
\hline$\Sigma_{s_{1}}$ & .04288 & .04393 & .04465 & .04515 & .04554 & .04585 & .04612 \\
\hline$d$ & 1. 165 & 1.070 & 1.038 & 1.032 & 1.037 & 1.048 & 1.061 \\
\hline$d_{1}$ & 3.033 & 3.079 & 3.112 & 3.135 & 3.153 & 3. 168 & 3.180 \\
\hline$d_{2}$ & .3131 & .4078 & .4919 & .5597 & .6170 & .6665 & .7101 \\
\hline$L_{1}^{2}$ & 32.29 & 32.22 & 32.16 & 32.11 & 32.07 & 32.03 & 31.99 \\
\hline$L \frac{2}{2}$ & 7.518 & 13.21 & 19.65 & 25.91 & 31.99 & 37.91 & 43.67 \\
\hline$p$ & .9730 & .9798 & .9834 & .9856 & .9871 & .9881 & .9890 \\
\hline
\end{tabular}

COMPOSITION GY WEIGHT FRACTION:

REFERENCE:

H $\quad .1119$

O .8881

material: Heavy Water

ASSUMED DENSITY: $\quad 1.105 \mathrm{gm} / \mathrm{cm}$

\begin{tabular}{|c|c|c|c|c|c|c|c|}
\hline TEMPERATURE: & $\infty F$ & $3000 \mathrm{~F}$ & $1000 \mathrm{~F}$ & $15000 \mathrm{~F}$ & $20000 \mathrm{~F}$ & $2500 \mathrm{~F}$ & $9000 \mathrm{~F}$ \\
\hline$D_{1}$ & 1.305 & 1.313 & 1.317 & 1.321 & 1.323 & 1.325 & 1.326 \\
\hline$D_{2}$ & .9580 & 1.040 & 1.093 & 1.127 & 1.151 & 1.169 & 1.183 \\
\hline$\Sigma_{a_{1}}$ & $3.040 \times 10^{-6}$ & $2.415 \times 10^{-6}$ & $2.053 \times 10^{-6}$ & $1.834 \times 10^{-6}$ & $1.674 \times 10^{-6}$ & $1.557 \times 10^{-6}$ & $1.464 \times 10^{-6}$ \\
\hline$\Sigma_{a_{2}}$ & .00003323 & .00002459 & .00001994 & .00001728 & .00001529 & .00001396 & .00001263 \\
\hline & & & & & & & \\
\hline$\Sigma_{s_{2}}$ & .009466 & .009693 & .009871 & .01001 & .01012 & .01021 & .01029 \\
\hline$d$ & 2.045 & 2.218 & 2.331 & 2.403 & 2.454 & 2.492 & 2.522 \\
\hline$d_{1}$ & 2. 782 & 2.798 & 2.808 & 2.816 & 2.820 & 2.824 & 2.826 \\
\hline$d_{2}$ & 2.042 & 2.217 & 2.330 & 2.402 & 2.453 & 2.492 & 2.522 \\
\hline$L_{1}^{2}$ & 137.8 & 135.4 & 133.4 & 132.0 & 130.8 & 129.7 & 128.8 \\
\hline$L \frac{2}{2}$ & 28830 . & 42300 . & 54840. & 65220. & 75290. & 83760. & 93700. \\
\hline$p$ & .9997 & .9998 & .9998 & .9998 & .9998 & .9998 & .9999 \\
\hline
\end{tabular}

COMPOSITION BY WEIGHT FRACTION:
D . .2007

O . .7993
DENSITY VARIATIONS AND REFERENCE: HCP $.998 \mathrm{gm} / \mathrm{cm}^{3}$ 


\begin{tabular}{|c|c|c|c|c|c|c|c|}
\hline TEMPERATURE: & $\cos F$ & $500 \mathrm{~F}$ & $1000 \mathrm{~F}$ & $1500 \mathrm{~F}$ & $2000 \mathrm{~F}$ & $2500 \mathrm{~F}$ & $3000 \mathrm{~F}$ \\
\hline$D_{1}$ & 1.755 & 1.755 & 1.756 & 1.756 & 1.756 & 1.756 & 1.756 \\
\hline$D_{2}$ & 1.524 & 1.585 & 1. 620 & 1.641 & 1.655 & 1.666 & 1.675 \\
\hline$\Sigma_{a_{1}}$ & $* \quad .0003777$ & .0003739 & .0003701 & .0003666 & .0003634 & .0003604 & .0003577 \\
\hline$\Sigma_{a_{2}}$ & .03225 & .02391 & .01939 & .01673 & .01493 & .01362 & .01259 \\
\hline$\Sigma_{s_{1}}$ & $5.134 \times 10^{-6}$ & $9.019 \times 10^{-6}$ & .00001285 & .00001649 & .00001979 & .00002284 & .00002570 \\
\hline$d$ & 3.742 & 3.742 & 3.742 & 3.742 & 3.742 & 3.742 & 3.742 \\
\hline$d_{1}$ & 3.742 & 3.742 & 3.742 & 3.742 & 3.742 & 3.742 & 3.742 \\
\hline$d_{2}$ & 3.249 & 3.378 & 3.452 & 3.497 & 3.528 & 3.552 & 3.570 \\
\hline$L \frac{2}{1}$ & 4585. & 4585. & 4584. & 4583. & 4582. & 4581 & 4580. \\
\hline$L \frac{2}{2}$ & 47.27 & 66.27 & 83.53 & 98.04 & 110,8 & 122.4 & 133.0 \\
\hline p & .01341 & .02355 & .03354 & .04304 & 05166 & $.05960^{\circ}$ & .06703 \\
\hline
\end{tabular}

COMPOSITION BY WEIGHT FRACTION:

$$
\text { Y } 1.00
$$

REFERENCE:
DENSTTY VARIATIONS AND REFERENCE:

ANPD: E. A. Aitken

$4.472 \mathrm{gm} / \mathrm{cm}^{3}$

MATERIAL: Zirconium

ASSUMED DENSITY: $\quad 6.50 \mathrm{gm} / \mathrm{cm}^{\circ}$

\begin{tabular}{|c|c|c|c|c|c|c|c|}
\hline TEMPẸRATURE: & $600 \mathrm{~F}$ & $500 \mathrm{~F}$ & $1000{ }^{\circ} \mathrm{F}$ & $1500^{\circ} \mathrm{F}$ & $2000{ }^{\circ} \mathrm{F}$ & $2500 \mathrm{~F}$ & $30000^{\circ}$ \\
\hline$D_{2}$ & 1.211 & 1.211 & 1.210 & 1.210 & 1.210 & 1.210 & 1.209 \\
\hline$D_{2}$ & 1.265 & 1.259 & 1.258 & 1.258 & 1.258 & 1.257 & 1.258 \\
\hline$\Sigma_{a_{1}}$ & .0003251 & .0002957 & .0002724 & .0002562 & .0002433 & .0002327 & .0002238 \\
\hline$\Sigma_{a_{2}}$ & .006852 & .005083 & .004121 & .003555 & .003173 & .002893 & .002675 \\
\hline$\Sigma_{s_{1}}$ & .00008989 & .0001217 & .0001473 & .0001657 & .0001804 & .0001926 & .0002031 \\
\hline$d$ & 2.582 & 2.583 & 2.583 & 2.584 & 2.584 & 2.585 & 2.585 \\
\hline$d_{1}$ & 2.581 & 2.580 & 2.580 & 2.579 & 2.579 & 2.578 & 2.578 \\
\hline$d_{2}$ & 2.695 & 2.685 & 2.682 & 2.681 & 2.680 & 2.680 & 2.680 \\
\hline$L_{1}^{2}$ & 2917. & 2900. & 2883. & 2868 . & 2855 . & 2844. & 2833. \\
\hline$L_{2}^{2}$ & 184.6 & 247.8 & 305.3 & 353.9 & 396.4 & 434.6 & 470.2 \\
\hline 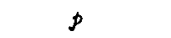 & .2166 & .2916 & .3509 & .3927 & .4257 & .4528 & .4758 \\
\hline
\end{tabular}

COMPOSITION BY WEIGHT FRACTION:

$$
\mathrm{Zr} \quad 1.00
$$

REFERENCE:
DENSITY VARIATIONS AND REFERENCE

ANPD: C. R. Simmons, $19576.50 \mathrm{gm} / \mathrm{cm}^{3}$

(measured)

ANPD: E. A. Aitken, $1956 \quad 6.52 \mathrm{gm} / \mathrm{cm}^{3}$ HCP $\quad 6.44 \mathrm{gm} / \mathrm{cm}^{3}$ RH $6.4 \mathrm{gm} / \mathrm{cm}^{3}$ 


\section{COMPARISON WITH EXPERIMENT}

As a matter of theoretical interest the age $L_{1}^{2}$ calculated by Program $\mathrm{C}_{2}$-Short has been compared to experimental age measurements for various materials for both the modified age theory and the Coveyou-Macauley slowing-down models. The comparison is given in Table 1 . The equations used with Coveyou-Macauley theory are identical with those of the modified age theory, with the exception of the relation between $q$ and $\phi$ in equation (2). This relationship is replaced by:

$$
\phi(u)=\frac{\frac{\Sigma_{s}(u)}{\xi \Sigma_{s}(u)} q(u)+S(u)}{\Sigma_{s}(u)+\Sigma_{a}(u)+D(u) K^{2}(u)} .
$$

On theoretical grounds one would expect that Coveyou-Macauley theory would be more accurate than modified age theory for moderators of high atomic we ight and considerably less accurate for moderation by hydrogen. On the other hand modified age theory treats the hydrogen slowing down more or less exactly, but treats the slowing down that results from other moderating materials in an approximation somewhat less accurate than conventional age theory.

Most of the experimental ages are quoted to the indium resonan ce of $1.4 \mathrm{ev}$. In order to get a good comparison between theory and experiment the age from indium to thermal $L_{(1.4 \mathrm{ev} \rightarrow \mathrm{Th})}^{2}$ was calculated as follows from Program $C_{2}$-Short and subtracted from $L_{1}^{2}$ :

$$
\begin{aligned}
& \phi_{(1.4 \mathrm{ev} \rightarrow \mathrm{Th})}=\operatorname{Sum}_{n=14}\left(\phi_{n}+\phi_{n+1}\right) \frac{U_{n}}{2} \\
& \Sigma_{(1.4 \mathrm{ev} \rightarrow \mathrm{Th})}=\frac{1}{\phi_{(1.4 \mathrm{ev} \rightarrow \mathrm{Th})}} \operatorname{Sum}_{n=14}^{n=t}\left(\Sigma_{a_{n}}+\Sigma_{a_{n+1}}\right) \frac{U_{n}}{2} \\
& \Sigma_{s_{\mathrm{Th}}}=\frac{q_{t}}{\phi_{(1.4 \mathrm{ev} \rightarrow \mathrm{Th})}} \cdot \\
& D_{(1.4 \mathrm{ev} \rightarrow \mathrm{Th})}=\frac{\operatorname{Sum}_{n=t}\left(L_{n}+L_{n+1}\right) \frac{U_{n}}{2}}{\phi_{(1.4 \mathrm{ev} \rightarrow \mathrm{Th})}^{\left(K^{2}\right)}} \\
& L_{1(1.4 \mathrm{ev} \rightarrow \mathrm{Th})}^{2}=\frac{D_{(1.4 \mathrm{ev} \rightarrow \mathrm{Th})}}{\Sigma_{a_{(1.4 \mathrm{ev} \rightarrow \mathrm{Th})}+\Sigma_{s_{\mathrm{Th}}}}} .
\end{aligned}
$$

An examination of Table 1 shows that in general the modified age theory gives better correlation with experimental data than Coveyou-Macauley theory for a $U^{235}$ fission source. -However the reverse is true for the Po-Be and $\mathrm{Ra}-\mathrm{Be}$ sources. For engineering analys is the modified age theory is recommended with the present lethargy divisions of Program $\mathrm{C}_{2}$ and with the present fission spectrum. The spectra used for the Po-Be, Ra-Be, and $U^{235}$ sources are experimental data that were normalized to a trapezoidally integrat ed area of unity for curves of the relative source strength as a function of lethargy $S(u)$ versus lethangy $u$. 
TABLE 1

COMPARISON OF EXPERIMENTAL AND THE ORETICAL AGES

\begin{tabular}{|c|c|c|c|c|c|c|c|}
\hline \multirow[b]{2}{*}{ Material Mixture by Volume } & \multirow[b]{2}{*}{$\begin{array}{l}\text { Experime ntal } \\
\text { Age, } \mathrm{cm}^{2}\end{array}$} & \multicolumn{2}{|c|}{$\begin{array}{c}\text { Corresponding Theoretical } \\
\text { Age, } \mathrm{cm}^{2}\end{array}$} & \multicolumn{2}{|c|}{$\begin{array}{l}\text { Theoretical Age to } \\
\text { Thermal Energv, } \mathrm{cm}^{2}\end{array}$} & \multicolumn{2}{|l|}{-} \\
\hline & & $\begin{array}{l}\text { Modified Age } \\
\text { Theory }\end{array}$ & $\begin{array}{c}\text { Coveyou- } \\
\text { Macauley } \\
\text { Theory }\end{array}$ & $\begin{array}{l}\text { Modified Age } \\
\text { Theory }\end{array}$ & $\begin{array}{c}\text { Coveyou- } \\
\text { Macauley } \\
\text { Theory }\end{array}$ & Source & Reference $^{\mathrm{a}}$ \\
\hline $\mathrm{Al} / \mathrm{H}_{2} \mathrm{O}=1 / 2$ & $r_{\text {In }}=49.6$ & 49.53 & 41.23 & 51.76 & 43.46 & $\mathrm{U}_{225}^{235}$ & 8 \\
\hline $\mathrm{Al} / \mathrm{H}_{2} \mathrm{O}=1$ & $r_{\text {In }}^{\ln }=76.8$ & 69.10 & 55.53 & 72.85 & 59.29 & $\mathrm{u}^{235}$ & 8 \\
\hline $\operatorname{Be}\left(\rho=1.85 \mathrm{gm} / \mathrm{cm}^{3}\right)$ & $\tau_{\operatorname{In}}^{\ln }=80.2 \pm 2.0$ & 85.32 & 68.05 & 99.60 & 82.37 & $\Sigma$ pile & 1 \\
\hline $\mathrm{Bi} / \mathrm{H}_{2} \mathrm{O}=1 / 2$ & ${ }_{T_{\text {In }}}^{\ln }=66.3$ & 79.53 & 61.45 & 81.57 & 63.49 & $\mathrm{Ra}-\mathrm{Be}$ & 7 \\
\hline $\mathrm{Bi} / \mathrm{H}_{2} \mathrm{O}=1$ & $\tau_{\text {In }}=79.5$ & 98.4 & 73.29 & 101.6 & 76.49 & $\mathrm{Ra}-\mathrm{Be}$ & 7 \\
\hline $\mathrm{Bi} / \mathrm{H}_{2} \mathrm{O}=2$ & $\tau_{\text {In }}^{1 \mathrm{n}}=86.2$ & 140.5 & 102.4 & 146.3 & 108.2 & $\mathrm{Ra}-\mathrm{Be}$ & 7 \\
\hline$C\left(\rho=1.6 \mathrm{gm} / \mathrm{cm}^{3}\right)$ & $\tau_{\text {In }}^{\ln }=311 \pm 3$ & 358.4 & 289.0 & 419.3 & 350.0 & $\mathrm{u}^{235}$ & 8 \\
\hline $0.998 \mathrm{D}_{2} \mathrm{O}+0.002 \mathrm{H}_{2} \mathrm{O}$ & $\tau_{\text {In }}^{10}=109$ & 112.9 & 106.1 & 136.1 & 129.3 & Enriched U & 10 \\
\hline $0.948 \mathrm{D}_{2} \mathrm{O}+0.052 \mathrm{H}_{2} \mathrm{O}$ & $r_{\text {In }}=93$ & 89.6 & 83.22 & 105.3 & 98.97 & Enriched U & 10 \\
\hline $0.910 \mathrm{D}_{2} \mathrm{O}+0.090 \mathrm{H}_{2} \mathrm{O}$ & $\tau_{\text {In }}^{10}=78$ & 77.88 & 71.76 & 90.15 & 84.06 & Enriched U & 10 \\
\hline $\mathrm{Fe} / \mathrm{H}_{2} \mathrm{O}^{2}=1 / 3$ & $r_{\text {In }}^{\ln }=52.3$ & 64.39 & 50.02 & 65.67 & 51.31 & Ra-Be & 3 \\
\hline $\mathrm{Fe} / \mathrm{H}_{2} \mathrm{O}=1 / 2$ & $r_{\text {In }}^{\text {In }}=56.0$ & 66.33 & 50.19 & 67.72 & 51.59 & $\mathrm{Ra}-\mathrm{Be}$ & 3 \\
\hline $\mathrm{Fe} / \mathrm{H}_{2} \mathrm{O}=1$ & $r_{\text {In }}^{\ln }=57.9$ & 75.88 & 55.16 & 77.59 & 56.91 & $\mathrm{Ra}-\mathrm{Be}$ & 3 \\
\hline $\mathrm{H}_{2} \mathrm{O}$ (distilled) & $\begin{array}{l}{ }^{r} T_{h}=56.6 \\
\tau_{T h}=56.3\end{array}$ & $\left\{\begin{array}{l}78.91 \\
77.85\end{array}\right.$ & $\left\{\begin{array}{l}68.61 \\
67.55\end{array}\right.$ & 78.91 & 68.61 & Po-Be & 4 \\
\hline $\mathrm{H}_{2} \mathrm{O}$ & $\tau_{\text {In }}=30.03$ & 31.23 & 28.15 & 32.29 & 29.21 & $\mathrm{u}^{235}$ & 6 \\
\hline $\mathrm{H}_{2} \mathrm{O}$ & $M^{2}=66.3$ & 86.43 & 76.13 & 78.91 & 68.61 & Po-Be & 2 \\
\hline $\mathrm{H}_{2} \mathrm{O}$ (distilled) & $\tau_{T h}=59.29$ & 71.02 & -62.05 & 71.02 & 62.05 & $\mathrm{Ra}-\mathrm{Be}$ & 5 \\
\hline $\mathrm{H}_{2} \mathrm{O}$ (distilled) & $\tau_{T h}^{T h}=58.9$ & 7102 & 62.05 & 71.02 & 62.05 & Ra-Be & 4 \\
\hline $\mathrm{Pb} / \mathrm{H}_{2} \mathrm{O}=1$ & $i_{\text {In }}^{1 \mathrm{n}}=72.8$ & 87.22 & 63.99 & 90.09 & 66.86 & $\mathrm{Ra}-\mathrm{Be}$ & 7 \\
\hline $\mathrm{SiO}_{2}\left(\rho=1.61 \mathrm{gm} / \mathrm{cm}^{3}\right)$ & $\tau_{I_{n}}^{\ln }=1900 \pm 90$ & 2623 & 2162 & 3082 & 2637 & $\mathrm{Ra}-\mathrm{Be}$ & 9 \\
\hline $0.194 \mathrm{Zr}+0.01 \mathrm{Hf}+0.796 \mathrm{H}_{2} \mathrm{O}$ & $\tau_{\mathrm{In}}^{\ln }=35.7$ & 34.30 & 29.00 & 35.36 & 30.11 & $\mathrm{u}^{235}$ & 6 \\
\hline $\mathrm{Z}_{\mathrm{r}} / \mathrm{H}_{2} \mathrm{O}=1 / 4$ & $M^{2}=77.5$ & 91.62 &, 75.57 & 81.13 & 65.08 & Po-Be & 2 \\
\hline $\mathrm{Zr} / \mathrm{H}_{2} \mathrm{O}=1 / 2$ & $\mathrm{M}^{2}=87.5$ & 101.67 & 81.37 & 88.38 & 67.78 & Po-Be & 2 \\
\hline$\dot{\mathrm{Z}}_{\mathrm{r}} / \mathrm{H}_{2} \mathrm{O}=1$ & $M^{2}=107.9$ & 126.7 & 97.99 & 106.8 & 78.09 & Po-Be & 2 \\
\hline
\end{tabular}

${ }^{a} \dot{A}$ list of references is given in section 6 . 


\section{REFERENCES}

1. “Argonne National Laboratory Report for July, August, September, 1947," ANL-4076, Lemont, Illinois.

2. Baer, W., "Spatial Distribution of Thermal Neutrons from a Polonium-Beryllium Source in WaterZirconium Mixtures," West inghouse Electric Corporation Atomic Power Division, WAPD-99, Decemb er 1953.

3. Fieno, D., "Slowing-Down Distribution to Indium Resonance of Neutrons from a Ra-a-Be Source in Water-Iron Mixtures," National Advisory Committee for Aeronautics, NACA-RM E54HO4, November 1, 1954, p. 9.

4. Graham, R. H., Donaldson, J. R., Fitch, S. H., Flora, J. W., and Nather, R. E., "Migration Area of Polonium-Beryllium Neutrons in Water," California Research and Development Company Livermore Laboratory, MTA-45, May 13, 1953.

5. Flowers, B. H., Graham, G. A. R., Pontecorvo, B., and Seligman, H., "Migration Length of Neutrons in Water Plus Uranium Metal Systems," National Research Council of Canada Montreal Laboratory, MP-154, July 20, 1945.

6. Hoover, J. I. and Arnette, T. I., "The Resonance Integral of Zirconium ( $\sim 1 \%$ Hafnium) and the Slowing Down Length in a Mixture (4:1 Volume) of Light Water and Zirconium Metal," Oak Ridge National Laboratory, ORNL-641, July 27, 1950.

7. Munn, A. M. and Pontecorvo, B., "Spatial Distribution of Neutrons in Hydrogenous Media Containing Bismuth, Lead, and Iron," Canadian Journal of Res earch, Volume 25, No. 3, Section A, National Research Council of Canada, 1957.

8. The Reactor Handbook, Vol. 1 Physics, U.S. Atom ic Energy Commission, RH-1, June 1953.

9. Tittman, Jay, “Neutron Moderation in $\mathrm{SiO}_{2}$," Physical Review, 95, American Institute of Physics, Incorporated, 1954.

10. Wade, J. W., "Neutrot Age in Mixtures of Light and Heavy Water," De Pont de Nemours (E. I.) and Co., DP-163, June 1956.

11. "Nuclear Codes Group Newsletter No. 4," Atomic Energy Commission Computing Facility, Institute of Mathematical Sciences, New York Univers ity, June 1, 1957.

12. Selengut, David, "Critical Mass Calculations for Bare Hydrogen Moderated Reactors By Means of Transport Theory," Aircraft Nuclear Propulsion Department, General Electric Company, APEX 1'21, September 1952 .

13. Wachspress, E. L., "Generalized Two-Space Dimension Multigroup Coding for the IBM 704," KAPL-1724, April 30, 1957. 